\title{
Empirical Bayes PCA in high dimensions
}

\author{
Xinyi Zhong* Chang $\mathrm{Su}^{*} \quad$ Zhou Fan
}

\begin{abstract}
When the dimension of data is comparable to or larger than the number of data samples, Principal Components Analysis (PCA) may exhibit problematic high-dimensional noise. In this work, we propose an Empirical Bayes PCA method that reduces this noise by estimating a joint prior distribution for the principal components. EB-PCA is based on the classical KieferWolfowitz nonparametric MLE for empirical Bayes estimation, distributional results derived from random matrix theory for the sample PCs, and iterative refinement using an Approximate Message Passing (AMP) algorithm. In theoretical "spiked" models, EB-PCA achieves Bayesoptimal estimation accuracy in the same settings as an oracle Bayes AMP procedure that knows the true priors. Empirically, EB-PCA significantly improves over PCA when there is strong prior structure, both in simulation and on quantitative benchmarks constructed from the 1000 Genomes Project and the International HapMap Project. An illustration is presented for analysis of gene expression data obtained by single-cell RNA-seq.
\end{abstract}

\section{Introduction}

Principal components analysis (PCA) is a widely used technique for dimensionality reduction. However, when the dimension of the data may be comparable to or larger than the number of available data samples, it is known that the sample principal components (PCs) may exhibit phenomena of high-dimensional noise [Lu02, JL09]. We propose a method called EB-PCA for reducing this noise, using the classical statistical idea of empirical Bayes [Rob56, Efr12].

Figure 1 illustrates EB-PCA on a genetics example. Panel (a) displays the top 4 PCs of a genotype matrix from the 1000 Genomes Project [The15], containing genotypes of 2504 individuals at 100,000 common single nucleotide polymorphisms (SNPs). The PCs depict the stratification of these individuals according to five broad ethnic populations. Here, the number of SNPs far exceeds the dimension 2504 of each PC, and the estimation noise is small. This allows us to interpret the PCs in panel (a) as an approximate "ground truth".

The phenomenon of high-dimensional noise is illustrated in panel (b), which displays the top 4 PCs for genotypes of the same 2504 individuals subsampled at only 1000 randomly selected SNPs. Applying EB-PCA to this reduced data of 1000 SNPs yields the PC estimates displayed in panel (c). These are remarkably close to the PCs in panel (a) computed on all 100,000 SNPs, even though EB-PCA has only access to the 1000 subsampled SNPs. In Section 4, we use this subsampling approach to demonstrate a sizeable quantitative improvement of EB-PCA over PCA. We also illustrate an application to single-cell RNA-seq gene expression data where a ground truth is unknown.

${ }^{*}$ These authors contributed equally.

XZ: Yale University, Department of Statistics and Data Science. xinyi.zhong@yale.edu

CS: Yale University, Department of Biostatistics. c.su@yale.edu

ZF: Yale University, Department of Statistics and Data Science. zhou.fan@yale.edu 
(a)
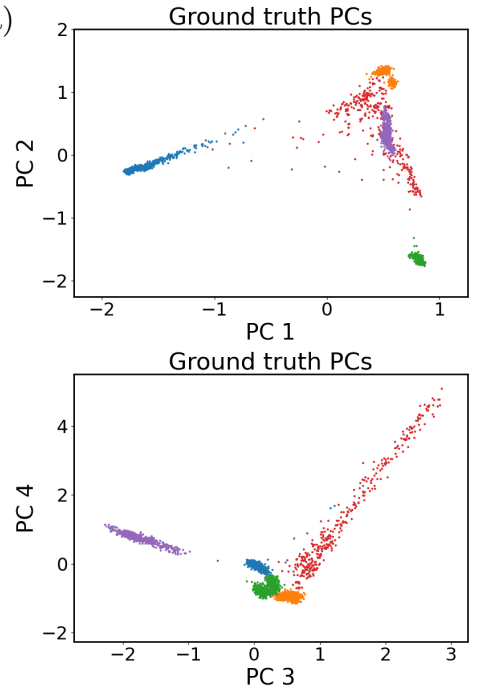

(b)
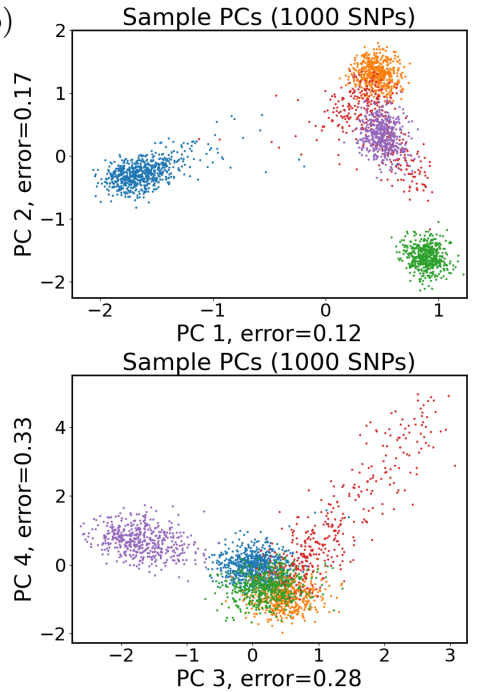

(c)

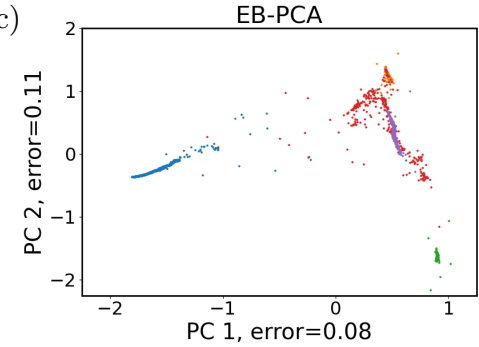

EB-PCA

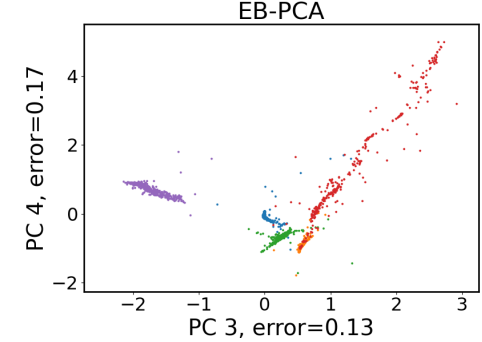

- South Asian

Figure 1: Illustration of EB-PCA on genotype data from the 1000 Genomes Project. (a) 1st vs. 2nd PC and 3rd vs. 4th PC, for the genotypes of 2504 individuals across 100,000 common SNPs. Each scatterplot has 2504 data points, representing the embedding of these individuals into a 4-dimensional space, with points colored by the individuals' ethnicity. We take these PCs as the ground truth. (b) PCs computed from a random subsample of 1000 SNPs. Substantial high-dimensional noise is observed in these PCs. (c) The EB-PCA estimates of the top 4 PCs, computed from the same subsampled data as in panel (b). These estimates are much closer to the ground-truth PCs in panel (a) and have quantitatively lower estimation error.

A central component of the method is a Bayes Approximate Message Passing (AMP) procedure [RF12, MV21] that implements approximate Bayesian inference for low-rank matrix estimation in high dimensions. EB-PCA adapts Bayes AMP, which requires knowledge of the true prior distributions, to more typical settings in practice where such information is unavailable, by nonparametrically estimating the priors from the sample PCs and the AMP iterates. Similar strategies can be applied to Bayes AMP algorithms for other applications.

To describe the main ideas behind EB-PCA, consider a rank-one signal-plus-noise model for the observed data,

$$
\mathbf{Y}=\frac{s}{n} \cdot \mathbf{u} \mathbf{v}^{\top}+\mathbf{W} \in \mathbb{R}^{n \times d}
$$

where $\mathbf{u} \in \mathbb{R}^{n}$ and $\mathbf{v} \in \mathbb{R}^{d}$ are the left and right true PCs of interest, with associated signal strength $s>0$, and $\mathbf{W} \in \mathbb{R}^{n \times d}$ is i.i.d. Gaussian observational noise. We discuss possible extensions to more general noise in Section 6 . We will refer to the leading left- and right-singular vectors $\mathbf{f} \in \mathbb{R}^{n}$ and $\mathbf{g} \in \mathbb{R}^{d}$ of $\mathbf{Y}$ as the sample PCs.

The EB-PCA approach consists of three main ideas, each of which is individually well-studied:

1. Kiefer-Wolfowitz NPMLE. Consider the classical compound decision problem of estimating $\boldsymbol{\theta} \in \mathbb{R}^{n}$ from a Gaussian observation vector $\mathbf{x} \sim \mathcal{N}\left(\mu \cdot \boldsymbol{\theta}, \sigma^{2} \cdot \operatorname{Id}_{n \times n}\right)$, for two known scalar parameters $\mu, \sigma^{2}>0$. The empirical Bayes paradigm first posits a prior distribution $\pi_{*}$ for the coordinates of $\boldsymbol{\theta}$, then estimates $\pi_{*}$ by an estimator $\pi$ based on the marginal density of the observed coordinates of $\mathbf{x}$, and finally applies Bayes's rule defined by $\pi$ to "denoise" $\mathbf{x}$ 
and obtain the estimate of $\boldsymbol{\theta}$.

A nonparametric implementation of this paradigm was described in [Rob50, KW56], which suggested estimating $\pi_{*}$ by the nonparametric maximum likelihood estimator (NPMLE) that maximizes the likelihood of $\mathbf{x}$ over all prior probability distributions $\pi$ on the real line. It was shown in [KW56, Lai78, Lin83a] that such a maximizer $\pi$ exists with discrete and finite support. We denote by

$$
\theta\left(\mathbf{x} \mid \mu, \sigma^{2}, \pi\right)=\mathbb{E}_{\pi}[\boldsymbol{\theta} \mid \mathbf{x}]
$$

the empirical Bayes posterior mean estimate of $\boldsymbol{\theta}$ using this estimated prior $\pi$.

2. Random matrix asymptotics for sample PCs. In the model of (1.1), an influential line of work [BBAP05, Pau07, Nad08, BGN12] has quantified the asymptotic error of the sample PCs $(\mathbf{f}, \mathbf{g})$ for the true PCs $(\mathbf{u}, \mathbf{v})$ when $n, d \rightarrow \infty$ simultaneously such that $d / n \rightarrow \gamma \in(0, \infty)$. This work showed that in this high-dimensional limit,

$$
\langle\mathbf{f}, \mathbf{u}\rangle \rightarrow \bar{\mu}_{*} \equiv \bar{\mu}_{*}(s, \gamma), \quad\langle\mathbf{g}, \mathbf{v}\rangle \rightarrow \mu_{*} \equiv \mu_{*}(s, \gamma)
$$

for two inner products $\mu_{*}, \bar{\mu}_{*} \in[0,1)$ that depend only on the signal strength $s$ and the dimension ratio $\gamma$. For $s$ larger than a certain phase transition threshold $s_{*}(\gamma)$, the leading singular value of $\mathbf{Y}$ emerges as an outlier from the bulk distribution of its remaining singular values, the inner products $\bar{\mu}_{*}, \mu_{*}$ are strictly positive, and $\mathbf{g}$ has an approximate entrywise Gaussian law

$$
\mathbf{g} \approx \mathcal{N}\left(\mu_{*} \cdot \mathbf{v}, \sigma_{*}^{2} \cdot \operatorname{Id}_{d \times d}\right), \quad \sigma_{*}^{2}=1-\mu_{*}^{2} .
$$

An analogous approximation holds for $\mathbf{f}$ and $\mathbf{u}$. This provides a connection to the compound decision problem above. EB-PCA estimates $\left(\mu_{*}, \sigma_{*}^{2}\right)$ by estimating $s$, and applies the KieferWolfowitz NPMLE to obtain an empirical Bayes estimate $\hat{\mathbf{v}}$ for $\mathbf{v}$.

3. Iterative refinement via AMP. If this estimate $\hat{\mathbf{v}}$ is more accurate than the original sample $\mathrm{PC} \mathbf{g}$ for $\mathbf{v}$, then we expect $\mathbf{Y} \hat{\mathbf{v}}$ to be more accurate than $\mathbf{Y g} \propto \mathbf{f}$ for $\mathbf{u}$. This suggests that empirical Bayes denoising should be applied to $\mathbf{Y} \hat{\mathbf{v}}$ instead of $\mathbf{f}$ to estimate $\mathbf{u}$, and leads to an iterative idea [WS21] of initializing $\mathbf{g}^{0}=\mathbf{g}$ and computing

$$
\begin{array}{ll}
\mathbf{v}^{t}=\theta\left(\mathbf{g}^{t} \mid \mu_{t}, \sigma_{t}^{2}, \pi_{t}\right), & \mathbf{f}^{t}=\mathbf{Y} \mathbf{v}^{t}, \\
\mathbf{u}^{t}=\theta\left(\mathbf{f}^{t} \mid \bar{\mu}_{t}, \bar{\sigma}_{t}^{2}, \bar{\pi}_{t}\right), & \mathbf{g}^{t+1}=\mathbf{Y}^{\top} \mathbf{u}^{t} .
\end{array}
$$

Here, $\pi_{t}, \bar{\pi}_{t}$ are nonparametrically estimated priors and $\mu_{t}, \sigma_{t}^{2}, \bar{\mu}_{t}, \bar{\sigma}_{t}^{2}$ are scalar parameters in each iteration. In the first iteration, $\mathbf{v}^{0}=\hat{\mathbf{v}}$ is the above empirical Bayes estimate of $\mathbf{v}$.

Unfortunately, this procedure does not ensure that $\left(\mathbf{f}^{t}, \mathbf{g}^{t}\right)$ have approximate entrywise Gaussian laws after this first iteration, breaking the connection to the compound decision problem in subsequent iterations. EB-PCA applies instead an AMP algorithm as developed in [RF12, MV21],

$$
\begin{array}{ll}
\mathbf{v}^{t}=\theta\left(\mathbf{g}^{t} \mid \mu_{t}, \sigma_{t}^{2}, \pi_{t}\right), & \mathbf{f}^{t}=\mathbf{Y} \mathbf{v}^{t}-b_{t} \mathbf{u}^{t-1}, \\
\mathbf{u}^{t}=\theta\left(\mathbf{f}^{t} \mid \bar{\mu}_{t}, \bar{\sigma}_{t}^{2}, \bar{\pi}_{t}\right), & \mathbf{g}^{t+1}=\mathbf{Y}^{\top} \mathbf{u}^{t}-\bar{b}_{t} \mathbf{v}^{t}
\end{array}
$$

The Onsager corrections $b_{t} \mathbf{u}^{t-1}$ and $\bar{b}_{t} \mathbf{v}^{t}$ are defined so as to remove a bias of $\left(\mathbf{f}^{t}, \mathbf{g}^{t}\right)$ in the directions of $\left(\mathbf{u}^{t-1}, \mathbf{v}^{t}\right)$ and restore the entrywise Gaussian approximations. 
EB-PCA is most effective when there is strong prior structure for the true PCs. We described the rank-one model of (1.1) for clarity, but in many examples including Figure 1, there is stronger structure jointly over several PCs. In these examples, we learn a joint prior in $k>1$ dimensions, where $k$ is the number of PCs to be simultaneously estimated. The result of Figure 1(c) is obtained by joint empirical Bayes estimation for all $k=4$ depicted PCs, rather than estimating each PC individually. We describe the method in more detail in Section 2.4 and present theoretical guarantees in Section 5.

This application of empirical Bayes methodology to PCA via an iterative algorithm is closely related to earlier and inspirational work by [WS21], who proposed an empirical Bayes matrix factorization (EBMF) method that yields the iterations of (1.2). EBMF is derived from a "naive mean-field" variational approximation to the posterior distribution of $(\mathbf{u}, \mathbf{v})$, and we discuss further in Section 2.5 the relation between EB-PCA and this naive mean-field approach.

\subsection{Related literature}

The possible inconsistency of PCA in high dimensions has been discussed in [Lu02, JL09, JP18], and improving PCA using prior structure has been a long-standing goal. A large body of literature has notably studied sparse PCA methods, which improve over PCA under sparsity assumptions [CJ95, JTU03, dGJL05, ZHT06, AW08, BJNP13, CMW13, FLM13, Ma13, VCLR13]. Figure 1 illustrates an example where the PCs indeed have strong prior structure, but this structure is not well-characterized by entrywise sparsity. We believe that such examples may be common across scientific applications, and this forms the primary motivation for our work.

EB-PCA is complementary to spectral shrinkage methods that preserve the sample PCs but shrink or truncate the singular values [CCS10, LW12, SN13, Nad14, Cha15, GD17]. These methods have been motivated in part by a perspective that, in the absence of prior structural knowledge about the PCs, "...it is reasonable to require that covariance matrix estimators be rotationequivariant [and have] the same eigenvectors as the sample covariance matrix" [LW12]. Our work stands contrary to this perspective, illustrating that empirical Bayes ideas can substantially improve over such equivariant procedures even without knowledge of prior structure, as long as some structure is present.

EB-PCA is an empirical Bayes implementation of the multivariate Bayes AMP algorithm described by [MV21]. AMP algorithms were first developed for CDMA, compressed sensing, and generalized linear model applications by [Kab03, DMM09, Ran11]. Empirical Bayes versions of AMP for compressed sensing and GLMs were studied by [VS11, $\mathrm{KMS}^{+} 12$, VS13, KRFU14], in univariate and parametric contexts that are different from the nonparametric perspective of our work.

AMP algorithms for PCA have been studied in a line of work including [RF12, MT13, DM14, MR15, LKZ15b, $\left.\mathrm{KKM}^{+} 16, \mathrm{DAM} 17\right]$. These algorithms originally required an informative initialization independent of $\mathbf{Y}$, and [MV21] provided the practical extension of initializing at the sample PCs. A related line of work [LKZ15a, BDM+16, Mio17, AK18, LM19, BM19] has explored more generally the limits of low-rank matrix estimation with Bayesian priors. In particular, $\left[\mathrm{DM} 14, \mathrm{BDM}^{+} 16, \mathrm{DAM} 17\right]$ showed in various Bayesian rank-one models that AMP algorithms can achieve the asymptotically optimal squared-error Bayes risk, which has been characterized in [LKZ15a, BDM ${ }^{+} 16$, Mio17, LM19]. A second motivation for our work is to bring this important body of statistical theory a step closer to statistical practice. Our results imply that AMP algorithms can achieve Bayes-optimal estimation even without knowledge of the true priors.

The initial step of EB-PCA relies on quantitative understanding of spectral behavior in spiked random matrix models [Joh01, BBAP05, BS06, BY08]. We assume in this work a Gaussian model, 
where the error of the sample singular vectors was first studied in [Pau07, Nad08]. Such results have been extended to non-Gaussian settings in [CDMFF11, KY13, KY14, BKYY16, Cap18, Din20], models with non-white noise in [Mes08, BGN11, BGN12, BY12], and more general asymptotic regimes in [JM09, SSM13, WF17]. Related distributional properties of singular vectors were recently studied in [CDM18, BDW21, BDWW20].

The Kiefer-Wolfowitz NPMLE was proposed in [Rob50, KW56]. Identifiability, existence and uniqueness, asymptotic consistency, and discreteness of the support were studied in [KW56, Sim76, Lai78, Jew82, Lin83a, Lin83b, LR93], and a detailed treatment of these topics is provided in [Lin95]. [GvdV01, Zha09, JZ09, SG20] studied the rate of convergence of the NPMLE and associated empirical Bayes estimator, and our analyses draw on their techniques. Recently, [PW20] showed that these results on estimation rates are connected also to the size of the discrete NPMLE support. Computing and approximating the NPMLE has been discussed in [BSL92, Böh99, LG08, KM14, FD16].

\section{The EB-PCA method}

\subsection{Model}

The EB-PCA algorithm is derived in the following rank- $k$ version of the model in (1.1),

$$
\mathbf{Y}=\frac{1}{n} \cdot \mathbf{U} S \mathbf{V}^{\top}+\mathbf{W}=\sum_{i=1}^{k} \frac{s_{i}}{n} \cdot \mathbf{u}_{i} \mathbf{v}_{i}^{\top}+\mathbf{W} \in \mathbb{R}^{n \times d}
$$

The columns of $\mathbf{U}=\left(\mathbf{u}_{1}, \ldots, \mathbf{u}_{k}\right) \in \mathbb{R}^{n \times k}$ and $\mathbf{V}=\left(\mathbf{v}_{1}, \ldots, \mathbf{v}_{k}\right) \in \mathbb{R}^{d \times k}$ are $k$ left and right principal components of interest, and $S=\operatorname{diag}\left(s_{1}, \ldots, s_{k}\right) \in \mathbb{R}^{k \times k}$ contains the signal strengths of these PCs. $\mathbf{W} \in \mathbb{R}^{n \times d}$ is observational noise, which we assume has entries $w_{i j} \stackrel{i i d}{\sim} \mathcal{N}(0,1 / n)$.

Remark 2.1. We write the noise variance of $w_{i j}$ for convenience as $1 / n$, rather than a more general $\tau^{2} / n$, to avoid carrying $\tau^{2}$ throughout our formulas. This is without loss of generality, as $\mathbf{Y}, S, \mathbf{W}$ may be rescaled by a common factor $\tau$. In practice, we estimate this noise variance and rescale the data to match this scaling. Given $\mathbf{Y}_{\text {obs }} \in \mathbb{R}^{n \times d}$, we may estimate its entrywise residual variance upon regressing out its top $k \mathrm{PCs}$,

$$
\hat{\tau}^{2}=d^{-1} \cdot\|\mathbf{R}\|_{F}^{2}, \quad \mathbf{R}=\mathbf{Y}_{\text {obs }}-\text { top } k \text { PCs of } \mathbf{Y}_{\text {obs }} .
$$

We then set $\mathbf{Y}=\mathbf{Y}_{\text {obs }} / \hat{\tau}$. Consistency of $\hat{\tau}^{2}$ is discussed in Appendix A.2.

We study this model in the high-dimensional limit $n, d \rightarrow \infty$ such that $k$ and $\gamma \equiv d / n$ are both fixed. It is helpful to keep in mind a Bayesian setting where the rows of $\mathbf{U}$ and $\mathbf{V}$ are generated according to two fixed prior probability distributions $\bar{\pi}_{*}$ and $\pi_{*}$ on $\mathbb{R}^{k}$ (although we will only require empirical convergence to these priors in the later theory). The goal of EB-PCA is then to estimate these two priors from the data $\mathbf{Y}$, and to use these estimated priors to perform Bayesian estimation of $\mathbf{U}$ and $\mathbf{V}$.

To fix the scaling of the PCs, we normalize $\bar{\pi}_{*}$ and $\pi_{*}$ to satisfy $\mathbb{E}_{U \sim \bar{\pi}_{*}}\left[U_{i}^{2}\right]=1$ and $\mathbb{E}_{V \sim \pi_{*}}\left[V_{i}^{2}\right]=$ 1 for all $i=1, \ldots, k$. This ensures

$$
n^{-1}\left\|\mathbf{u}_{i}\right\|_{2}^{2} \rightarrow 1, \quad d^{-1}\left\|\mathbf{v}_{i}\right\|_{2}^{2} \rightarrow 1 .
$$

We will also assume $\mathbb{E}_{U \sim \bar{\pi}_{*}}\left[U_{i} U_{j}\right]=0$ and $\mathbb{E}_{V \sim \pi_{*}}\left[V_{i} V_{j}\right]=0$ for all $1 \leq i \neq j \leq k$, so that

$$
n^{-1} \mathbf{u}_{i}^{\top} \mathbf{u}_{j} \rightarrow 0, \quad d^{-1} \mathbf{v}_{i}^{\top} \mathbf{v}_{j} \rightarrow 0,
$$


lending to the interpretations of $\mathbf{u}_{i}$ and $\mathbf{v}_{i}$ as the (orthogonal) principal components. Under these scalings, the $k$ singular values of $n^{-1} \mathbf{U} S \mathbf{V}^{\top}$ converge to the limits $\sqrt{\gamma} s_{1}>\ldots>\sqrt{\gamma} s_{k}$ and we make the simplifying assumption that these limit values are distinct. Note that we will not enforce the orthogonality conditions $\mathbb{E}_{U \sim \bar{\pi}}\left[U_{i} U_{j}\right]=0$ and $\mathbb{E}_{V \sim \pi}\left[V_{i} V_{j}\right]=0$ for the estimated priors in the later algorithm, but approximate orthogonality will automatically hold from initializing the algorithm at the sample PCs.

Turning to the sample PCs, let us write the best rank- $k$ approximation for $\mathbf{Y}$ as

$$
\frac{1}{n} \cdot \mathbf{F} \Lambda \mathbf{G}^{\top}=\sum_{i=1}^{k} \frac{\lambda_{i}}{n} \cdot \mathbf{f}_{i} \mathbf{g}_{i}^{\top} .
$$

Here, the columns of $\mathbf{F}=\left(\mathbf{f}_{1}, \ldots, \mathbf{f}_{k}\right) \in \mathbb{R}^{n \times k}$ and $\mathbf{G}=\left(\mathbf{g}_{1}, \ldots, \mathbf{g}_{k}\right) \in \mathbb{R}^{d \times k}$ are the top $k$ left and right singular vectors of $\mathbf{Y}$, normalized analogously with a sign convention so that for all $1 \leq i \neq j \leq k$,

$$
d^{-1}\left\|\mathbf{g}_{i}\right\|^{2}=n^{-1}\left\|\mathbf{f}_{i}\right\|^{2}=1, \quad \mathbf{u}_{i}^{\top} \mathbf{f}_{i} \geq 0, \quad \mathbf{v}_{i}^{\top} \mathbf{g}_{i} \geq 0, \quad d^{-1} \mathbf{g}_{i}^{\top} \mathbf{g}_{j}=n^{-1} \mathbf{f}_{i}^{\top} \mathbf{f}_{j}=0 .
$$

We set $\Lambda=\operatorname{diag}\left(\lambda_{1}, \ldots, \lambda_{k}\right)$. Then the $k$ largest singular values of $\mathbf{Y}$ are given by $\sqrt{\gamma} \lambda_{1} \geq \ldots \geq$ $\sqrt{\gamma} \lambda_{k}$.

Under this model, the following phase transition occurs for the leading $k$ sample singular values and singular vectors of $\mathbf{Y}$ [BBAP05, Pau07, BGN12]: setting $s_{*}(\gamma)=\gamma^{-1 / 4}$, for super-critical PCs such that $s_{i}>s_{*}(\gamma)$, we have

$$
\lim _{n, d \rightarrow \infty} \sqrt{\gamma} \cdot \lambda_{i}>\lambda_{+}, \quad \lim _{n, d \rightarrow \infty} n^{-1} \mathbf{f}_{i}^{\top} \mathbf{u}_{i}>0, \quad \lim _{n, d \rightarrow \infty} d^{-1} \mathbf{g}_{i}^{\top} \mathbf{v}_{i}>0
$$

where $\lambda_{+}=1+\sqrt{\gamma}$ is the upper edge of the "bulk distribution" of the noise singular values. Conversely, for sub-critical PCs such that $s_{i} \leq s_{*}(\gamma)$,

$$
\lim _{n, d \rightarrow \infty} \sqrt{\gamma} \cdot \lambda_{i}=\lambda_{+}, \quad \lim _{n, d \rightarrow \infty} n^{-1} \mathbf{f}_{i}^{\top} \mathbf{u}_{i}=0, \quad \lim _{n, d \rightarrow \infty} d^{-1} \mathbf{g}_{i}^{\top} \mathbf{v}_{i}=0 .
$$

Thus the $i^{\text {th }}$ sample singular value is absorbed into the bulk, and the sample PCs are nearly orthogonal to the true PCs. For notational and expositional clarity, we will assume

$$
s_{i}>s_{*}(\gamma) \quad \text { for all } i=1, \ldots, k,
$$

i.e. all $k$ of the leading PCs are super-critical. Our theoretical results may be extended to more general settings having both super-critical and sub-critical PCs, where EB-PCA is applied only to the super-critical PCs that have positive alignment with the truth.

Remark 2.2. If the rows of $\mathbf{U}$ are drawn from $\bar{\pi}_{*}=\mathcal{N}\left(0, \operatorname{Id}_{k \times k}\right)$, then the rows of $\sqrt{n} \cdot \mathbf{Y}$ marginalized over $\mathbf{U}$ are distributed as $\mathcal{N}(0, \Sigma)$ where

$$
\Sigma=\sum_{i=1}^{k} s_{i}^{2} \cdot \frac{\mathbf{v}_{i} \mathbf{v}_{i}^{\top}}{n}+\operatorname{Id}_{d \times d} .
$$

Thus $\mathbf{Y}^{\top} \mathbf{Y}$ follows the spiked covariance model introduced in [Joh01], and our results pertain also to estimating the spike eigenvectors of $\Sigma$. In this model, it would be reasonable to consider a version of EB-PCA that fixes $\bar{\pi}_{*}=\mathcal{N}(0$, Id $)$, only estimates $\pi_{*}$, and performs Bayesian estimation of $\mathbf{V}$ but not of $\mathbf{U}$. We will focus instead on the more general scenario where both $\mathbf{U}$ and $\mathbf{V}$ may have non-Gaussian structure, and describe EB-PCA for estimating both matrices. 


\subsection{Empirical Bayes for the multivariate compound decision problem}

Let $\pi_{*}$ be a probability distribution on $\mathbb{R}^{k}$. For two given matrices $M, \Sigma \in \mathbb{R}^{k \times k}$ where $\Sigma$ is symmetric positive-definite, consider the compound decision model

$$
\Theta \sim \pi_{*}, \quad X \mid \Theta \sim \mathcal{N}(M \cdot \Theta, \Sigma)
$$

for $\Theta, X \in \mathbb{R}^{k}$. We will denote the Bayes posterior mean estimate of $\Theta$ based on $X$ as

$$
\theta\left(X \mid M, \Sigma, \pi_{*}\right)=\mathbb{E}_{\pi_{*}}[\Theta \mid X] .
$$

Suppose now that $\pi_{*}$ is unknown, but belongs to a known class of probability distributions $\mathcal{P}$ over $\mathbb{R}^{k}$. In a model of $n$ i.i.d. samples $x_{1}, \ldots, x_{n}$ distributed according to (2.6), stacked as the rows of a matrix $\mathbf{X} \in \mathbb{R}^{n \times k}$, consider the maximum likelihood estimator

$$
\begin{aligned}
\pi & =\operatorname{MLE}(\mathbf{X} \mid M, \Sigma, \mathcal{P}) \\
& \equiv \underset{\pi \in \mathcal{P}}{\arg \max } \prod_{i=1}^{n} \int \frac{1}{(2 \pi)^{k / 2}|\Sigma|^{1 / 2}} \cdot \exp \left(-\frac{\left(x_{i}-M \cdot \theta_{i}\right)^{\top} \Sigma^{-1}\left(x_{i}-M \cdot \theta_{i}\right)}{2}\right) \mathrm{d} \pi\left(\theta_{i}\right) .
\end{aligned}
$$

This integral is the marginal Gaussian mixture density of $x_{i}$ in the model of (2.6), and the notation makes explicit the dependence of $\pi$ on the prior class $\mathcal{P}$. We will be interested primarily in nonparametric classes $\mathcal{P}$, and $\pi$ is a nonparametric maximum likelihood estimate (NPMLE) for $\pi_{*}$. In our implementation, we take $\mathcal{P}$ as the class of all probability distributions on $\mathbb{R}^{k}$, and approximate this class $\mathcal{P}$ using a discrete support by applying the "exemplar method" of [LG08]. See Appendix E for details.

Stacking $\theta_{1}, \ldots, \theta_{n}$ as the rows of $\boldsymbol{\Theta} \in \mathbb{R}^{n \times k}$, the model for $\mathbf{X}$ may be written as

$$
\mathbf{X}=\boldsymbol{\Theta} M^{\top}+\mathbf{Z} \Sigma^{1 / 2}, \quad \mathbf{Z} \in \mathbb{R}^{n \times k} \text { has i.i.d. } \mathcal{N}(0,1) \text { entries. }
$$

The NPMLE $\pi$ defines an empirical Bayes estimate of $\boldsymbol{\Theta}$, which applies the posterior mean function $\theta(\cdot)$ for the estimated prior $\pi$ row-wise to $\mathbf{X}$. We denote this by

$$
\theta(\mathbf{X} \mid M, \Sigma, \pi)=\mathbb{E}_{\pi}[\boldsymbol{\Theta} \mid \mathbf{X}]
$$

\subsection{Initial denoising of the sample PCs}

In the model of (2.1), as $n, d \rightarrow \infty$, the precise forms of the limits of the super-critical singular values $\sqrt{\gamma} \cdot \lambda_{i}$ and corresponding $\mathrm{PCs} \mathbf{f}_{i}, \mathbf{g}_{i}$ of $\mathbf{Y}$ are given by

$$
\begin{gathered}
\sqrt{\gamma} \cdot \lambda_{i} \rightarrow \sqrt{\left(\gamma s_{i}^{2}+1\right)\left(s_{i}^{2}+1\right) / s_{i}^{2}}, \\
n^{-1} \mathbf{f}_{i}^{\top} \mathbf{u}_{i} \rightarrow \bar{\mu}_{*, i} \equiv \sqrt{1-\bar{\sigma}_{*, i}^{2}}, \quad d^{-1} \mathbf{g}_{i}^{\top} \mathbf{v}_{i} \rightarrow \mu_{*, i} \equiv \sqrt{1-\sigma_{*, i}^{2}}, \\
\bar{\sigma}_{*, i}^{2}=\frac{1+s_{i}^{2}}{s_{i}^{2}\left(\gamma s_{i}^{2}+1\right)}, \quad \sigma_{*, i}^{2}=\frac{1+\gamma s_{i}^{2}}{\gamma s_{i}^{2}\left(s_{i}^{2}+1\right)} .
\end{gathered}
$$

(See Lemma A.3.) Setting

$$
\begin{aligned}
\bar{M}_{*}=\operatorname{diag}\left(\bar{\mu}_{*, 1}, \ldots, \bar{\mu}_{*, k}\right), \quad & M_{*}=\operatorname{diag}\left(\mu_{*, 1}, \ldots, \mu_{*, k}\right), \\
\bar{\Sigma}_{*}=\operatorname{diag}\left(\bar{\sigma}_{*, 1}^{2}, \ldots, \bar{\sigma}_{*, k}^{2}\right), & \Sigma_{*}=\operatorname{diag}\left(\sigma_{*, 1}^{2}, \ldots, \sigma_{*, k}^{2}\right),
\end{aligned}
$$


a consequence is that $\mathbf{F} \in \mathbb{R}^{n \times k}$ and $\mathbf{G} \in \mathbb{R}^{d \times k}$ have the Gaussian approximations

$$
\mathbf{F} \approx \mathbf{U} \bar{M}_{*}^{\top}+\overline{\mathbf{Z}} \bar{\Sigma}_{*}^{1 / 2}, \quad \mathbf{G} \approx \mathbf{V} M_{*}^{\top}+\mathbf{Z} \Sigma_{*}^{1 / 2}
$$

for large $n$ and $d$, where $\overline{\mathbf{Z}}, \mathbf{Z} \in \mathbb{R}^{n \times k}$ have i.i.d. $\mathcal{N}(0,1)$ entries. This relates the behavior of the sample PCs $\mathbf{F}$ and $\mathbf{G}$ to the multivariate compound decision model in (2.9).

As the true matrices $\bar{M}_{*}, M_{*}, \bar{\Sigma}_{*}, \Sigma_{*}$ are unknown, we replace them by consistent estimates to derive empirical Bayes estimators for $\mathbf{U}$ and $\mathbf{V}$ : observe that (2.10) implies each value $s_{i}^{2}$ may be consistently estimated by

$$
\hat{s}_{i}^{2}=\left(\gamma \lambda_{i}^{2}-(1+\gamma)+\sqrt{\left(\gamma \lambda_{i}^{2}-(1+\gamma)\right)^{2}-4 \gamma}\right) /(2 \gamma)
$$

These may be used to obtain plug-in estimators $\bar{M}, M, \bar{\Sigma}, \Sigma$ for $\bar{M}_{*}, M_{*}, \bar{\Sigma}_{*}, \Sigma_{*}$, which substitute $\hat{s}_{i}$ for $s_{i}$ in (2.10). This yields the initial empirical Bayes estimates of $\mathbf{U}$ and $\mathbf{V}$ given by

$$
\begin{array}{ll}
\bar{\pi}=\operatorname{MLE}(\mathbf{F} \mid \bar{M}, \bar{\Sigma}, \mathcal{P}), & \hat{\mathbf{U}}=\theta(\mathbf{F} \mid \bar{M}, \bar{\Sigma}, \bar{\pi}), \\
\pi=\operatorname{MLE}(\mathbf{G} \mid M, \Sigma, \mathcal{P}), & \hat{\mathbf{V}}=\theta(\mathbf{G} \mid M, \Sigma, \pi) .
\end{array}
$$

\subsection{Iterative refinement using AMP}

We now describe iterative refinement using an AMP algorithm, as discussed also in Appendix J of [MV21]. This may begin with either the estimate for $\mathbf{U}$ or $\mathbf{V}$-here, we begin with $\mathbf{V}$.

The algorithm takes the following form: let $u_{1}, u_{2}, \ldots: \mathbb{R}^{k} \rightarrow \mathbb{R}^{k}$ and $v_{1}, v_{2}, \ldots: \mathbb{R}^{k} \rightarrow \mathbb{R}^{k}$ be two arbitrary sequences of Lipschitz functions. Initialize $\mathbf{G}^{0}=\mathbf{G}$ as the right sample PCs, and compute for $t=0,1,2, \ldots$

$$
\begin{array}{rlrl}
\mathbf{V}^{t} & =v_{t}\left(\mathbf{G}^{t}\right) \\
\mathbf{U}^{t} & =u_{t}\left(\mathbf{F}^{t}\right) & \mathbf{F}^{t} & =\mathbf{Y} \mathbf{V}^{t}-\mathbf{U}^{t-1} \cdot \gamma\left\langle\mathbf{d} v_{t}\left(\mathbf{G}^{t}\right)\right\rangle^{\top} \\
\mathbf{G}^{t+1} & =\mathbf{Y}^{\top} \mathbf{U}^{t}-\mathbf{V}^{t} \cdot\left\langle\mathbf{d} u_{t}\left(\mathbf{F}^{t}\right)\right\rangle^{\top}
\end{array}
$$

Here $u_{t}\left(\mathbf{F}^{t}\right) \in \mathbb{R}^{n \times k}$ and $v_{t}\left(\mathbf{G}^{t}\right) \in \mathbb{R}^{d \times k}$ denote the applications of $u_{t}$ and $v_{t}$ row-wise to $\mathbf{F}^{t}$ and $\mathbf{G}^{t}, \mathrm{~d} u_{t}: \mathbb{R}^{k} \rightarrow \mathbb{R}^{k \times k}$ and $\mathrm{d} v_{t}: \mathbb{R}^{k} \rightarrow \mathbb{R}^{k \times k}$ denote Jacobian matrices of these functions, and $\left\langle\mathbf{d} u_{t}\left(\mathbf{F}^{t}\right)\right\rangle \in \mathbb{R}^{k \times k}$ and $\left\langle\mathbf{d} v_{t}\left(\mathbf{G}^{t}\right)\right\rangle \in \mathbb{R}^{k \times k}$ are the averages of $\mathbf{d} u_{t}$ and $\mathbf{d} v_{t}$ across the rows of $\mathbf{F}^{t}$ and $\mathbf{G}^{t}$.

Under the model of (2.1), Gaussian approximations analogous to (2.13) continue to hold for $\mathbf{F}^{t}$ and $\mathbf{G}^{t}$ across iterations, where

$$
\mathbf{F}^{t} \approx \mathbf{U} \bar{M}_{*, t}^{\top}+\overline{\mathbf{Z}} \bar{\Sigma}_{*, t}^{1 / 2}, \quad \mathbf{G}^{t} \approx \mathbf{V} M_{*, t}^{\top}+\mathbf{Z} \Sigma_{*, t}^{1 / 2} .
$$

Here $\bar{M}_{*, t}, M_{*, t}, \bar{\Sigma}_{*, t}, \Sigma_{*, t}$ are deterministic matrices that prescribe the parameters of the compound decision model associated to each iteration. In contrast to the initial state of (2.11)-(2.12), these matrices are no longer diagonal in later iterations, if the prior is a general multivariate distribution on $\mathbb{R}^{k}$. They evolve over iterations according to a state evolution

$$
\left(M_{*, 0}, \Sigma_{*, 0}\right) \mapsto\left(\bar{M}_{*, 0}, \bar{\Sigma}_{*, 0}\right) \mapsto\left(M_{*, 1}, \Sigma_{*, 1}\right) \mapsto\left(\bar{M}_{*, 1}, \bar{\Sigma}_{*, 1}\right) \mapsto \cdots
$$

given by the initializations $\left(M_{*, 0}, \Sigma_{*, 0}\right) \equiv\left(M_{*}, \Sigma_{*}\right)$ describing the sample PCs $\mathbf{G}^{0} \equiv \mathbf{G}$ in $(2.11)-$ (2.12), and by the updates

$$
\begin{array}{cl}
\bar{M}_{*, t}=\gamma \mathbb{E}\left[v_{t}\left(G_{t}\right) V^{\top}\right] S, & \bar{\Sigma}_{*, t}=\gamma \mathbb{E}\left[v_{t}\left(G_{t}\right) v_{t}\left(G_{t}\right)^{\top}\right], \\
M_{*, t+1}=\mathbb{E}\left[u_{t}\left(F_{t}\right) U^{\top}\right] S, & \Sigma_{*, t+1}=\mathbb{E}\left[u_{t}\left(F_{t}\right) u_{t}\left(F_{t}\right)^{\top}\right] .
\end{array}
$$


$S=\operatorname{diag}\left(s_{1}, \ldots, s_{k}\right)$ is the diagonal matrix of signal strengths in (2.1), and the expectations are over the random vectors

$$
U \sim \bar{\pi}_{*}, \quad F_{t}\left|U \sim \mathcal{N}\left(\bar{M}_{*, t} \cdot U, \bar{\Sigma}_{*, t}\right), \quad V \sim \pi_{*}, \quad G_{t}\right| V \sim \mathcal{N}\left(M_{*, t} \cdot V, \Sigma_{*, t}\right) .
$$

These laws of $F_{t}$ and $G_{t}$ approximate the row-wise distributions of $\mathbf{F}^{t}$ and $\mathbf{G}^{t}$.

If $\bar{\pi}_{*}, \pi_{*}$ and $\bar{M}_{*, t}, M_{*, t}, \bar{\Sigma}_{*, t}, \Sigma_{*, t}$ are all known, then applying this algorithm with the Bayes posterior mean functions

$$
u_{t}(\mathbf{X})=\theta\left(\mathbf{X} \mid \bar{M}_{*, t}, \bar{\Sigma}_{*, t}, \bar{\pi}_{*}\right), \quad v_{t}(\mathbf{X})=\theta\left(\mathbf{X} \mid M_{*, t}, \Sigma_{*, t}, \pi_{*}\right)
$$

implements an iterative variational Bayesian inference scheme [MV21]. We will call this the "oracle" Bayes AMP algorithm. For these $u_{t}, v_{t}$, we have the identities $\mathbb{E}\left[u_{t}\left(F_{t}\right) U^{\top}\right]=\mathbb{E}\left[u_{t}\left(F_{t}\right) u_{t}\left(F_{t}\right)^{\top}\right]$ and $\mathbb{E}\left[v_{t}\left(G_{t}\right) V^{\top}\right]=\mathbb{E}\left[v_{t}\left(G_{t}\right) v_{t}\left(G_{t}\right)^{\top}\right]$, so (2.19) yields

$$
\bar{M}_{*, t}=\bar{\Sigma}_{*, t} \cdot S, \quad M_{*, t+1}=\Sigma_{*, t+1} \cdot S .
$$

EB-PCA uses the posterior mean functions defined instead by NPMLEs of $\bar{\pi}_{*}$ and $\pi_{*}$, together with the empirical estimates $\bar{\Sigma}_{t}=n^{-1}\left(\mathbf{V}^{t}\right)^{\top} \mathbf{V}^{t}, \bar{M}_{t}=\bar{\Sigma}_{t} \hat{S}, \Sigma_{t+1}=n^{-1}\left(\mathbf{U}^{t}\right)^{\top} \mathbf{U}^{t}$, and $M_{t+1}=\Sigma_{t+1} \hat{S}$ where $\hat{S}=\operatorname{diag}\left(\hat{s}_{1}, \ldots, \hat{s}_{k}\right)$ is the estimate of $S$ from $(2.14)$. (For $\bar{\Sigma}_{t}$, we have applied $\gamma / d=1 / n$.) These empirical estimates avoid the need to perform Gaussian integrations to analytically evaluate the expectations that define the true matrices $\bar{M}_{*, t}, M_{*, t}, \bar{\Sigma}_{*, t}, \Sigma_{*, t}$. EB-PCA is initialized at the right sample $\mathrm{PCs} \mathbf{G}^{0}=\mathbf{G}$ and the plug-in estimates $\left(M_{0}, \Sigma_{0}\right) \equiv(M, \Sigma)$ from the preceding section. In particular, $\mathbf{V}^{0}$ is the initial empirical Bayes estimate for $\mathbf{V}$ based on $\mathbf{G}$ as previously described.

We summarize the full EB-PCA method as Algorithm 1.

Remark 2.3. In Lines 6 and 10 of Algorithm 1, we form new NPMLEs for $\bar{\pi}_{*}$ and $\pi_{*}$ in each iteration. This allows for the possibility of improving these estimates as the signal-to-noise ratios reflected by the state parameters $\left(M_{t}, \Sigma_{t}\right)$ and $\left(\bar{M}_{t}, \bar{\Sigma}_{t}\right)$ improve across iterations. In data examples with strong signal, this re-estimation of $\bar{\pi}_{*}$ and $\pi_{*}$ may be unneeded, and removed to improve computational efficiency. See Appendix E.2 for further discussion.

Remark 2.4. For the state evolution to correctly describe the AMP iterates under the PCA initialization $\mathbf{G}^{0}=\mathbf{G}$, the first AMP iteration for $\mathbf{F}^{0}$ in Line 8 should use $\mathbf{U}^{-1}=\mathbf{F} \cdot \Sigma_{0}^{1 / 2}$ as initialized in Line 4, rather than $\mathbf{U}^{-1}=0$ as described in [MV21]. We elaborate on this in Appendix C.

\subsection{Relation to naive mean field variational Bayes}

[WS21] propose an empirical Bayes matrix factorization (EBMF) algorithm similar to EB-PCA, based instead on naive mean-field variational Bayes: in the rank-one model of (1.1), this approximates the posterior law $p(\mathbf{u}, \mathbf{v} \mid \mathbf{Y})$ by a factorized form $\bar{q}(\mathbf{u}) q(\mathbf{v})=\prod_{i=1}^{n} \bar{q}_{i}\left(u_{i}\right) \prod_{j=1}^{d} q_{j}\left(v_{j}\right)$. The distributions $\bar{q}_{i}, q_{j}$ are chosen to minimize the Kullback-Leibler divergence $D_{\mathrm{KL}}(\bar{q}(\mathbf{u}) q(\mathbf{v}) \| p(\mathbf{u}, \mathbf{v} \mid \mathbf{Y}))$ or, equivalently, to maximize the evidence lower bound

$$
\mathcal{F}(\bar{q}, q)=\mathbb{E}_{\mathbf{u} \sim \bar{q}, \mathbf{v} \sim q}[\log p(\mathbf{Y}, \mathbf{u}, \mathbf{v})-\log \bar{q}(\mathbf{u}) q(\mathbf{v})] .
$$

Here, the joint density $p(\mathbf{Y}, \mathbf{u}, \mathbf{v})$ depends on the priors $(\bar{\pi}, \pi)$ for $(\mathbf{u}, \mathbf{v})$, and EBMF estimates these by maximizing $\mathcal{F}$ jointly over $(\bar{q}, \bar{\pi}, q, \pi)$. This maximization is performed via the iterative coordinate ascent variational inference (CAVI) updates

$$
\left(q_{t}, \pi_{t}\right)=\underset{q, \pi}{\arg \max } \mathcal{F}\left(\bar{q}_{t-1}, \bar{\pi}_{t-1}, q, \pi\right), \quad\left(\bar{q}_{t}, \bar{\pi}_{t}\right)=\underset{\bar{q}, \bar{\pi}}{\arg \max } \mathcal{F}\left(\bar{q}, \bar{\pi}, q_{t}, \pi_{t}\right)
$$




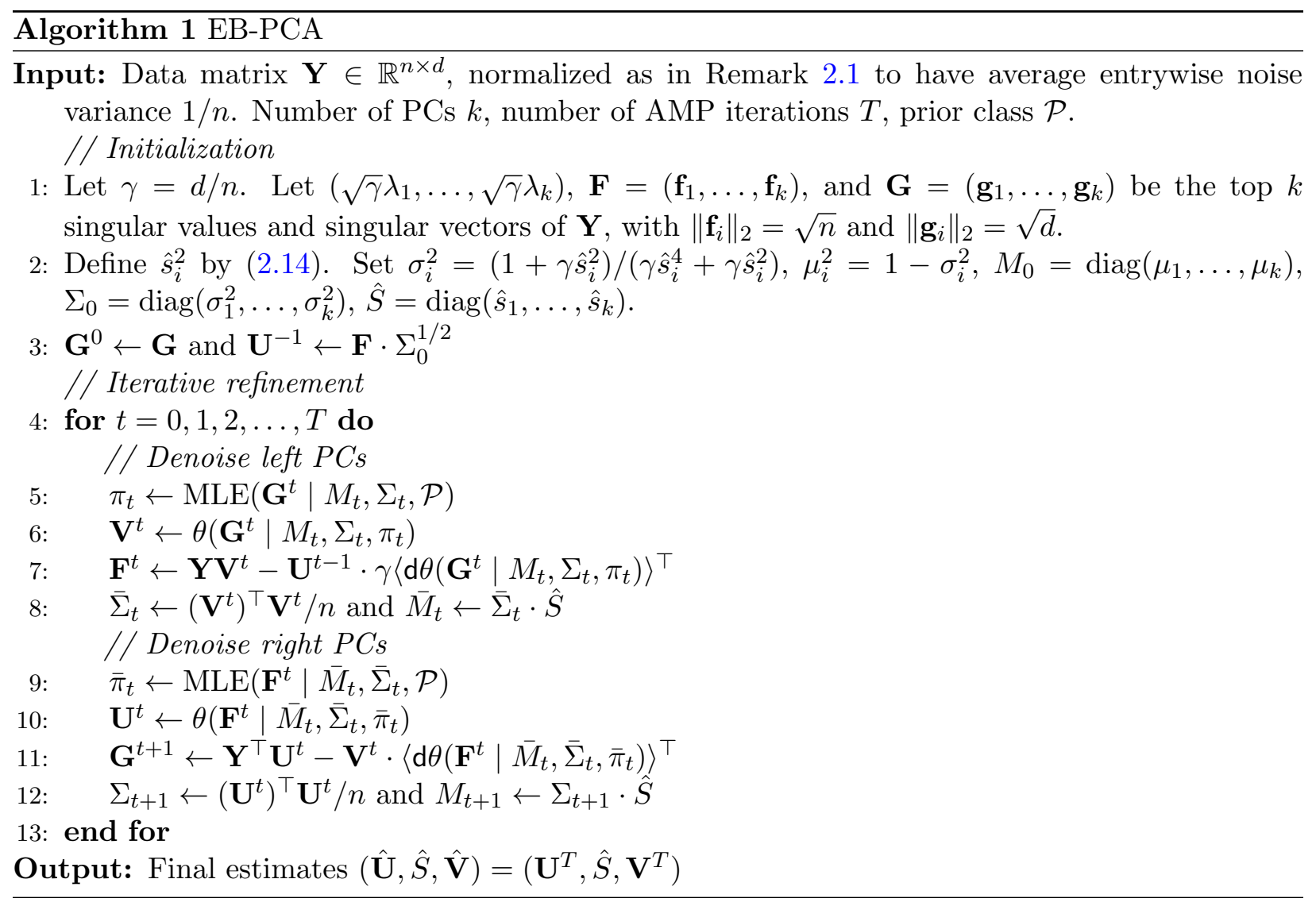

As shown in [WS21], the CAVI updates admit a simple form in terms of the quantities

$$
\mathbf{v}^{t} \equiv \mathbb{E}_{\mathbf{v} \sim q_{t}}[\mathbf{v}], \quad \bar{\sigma}_{t}^{2} \equiv n^{-1} \mathbb{E}_{\mathbf{v} \sim q_{t}}\left[\|\mathbf{v}\|^{2}\right], \quad \mathbf{u}^{t} \equiv \mathbb{E}_{\mathbf{u} \sim \bar{q}_{t}}[\mathbf{u}], \quad \sigma_{t+1}^{2} \equiv n^{-1} \mathbb{E}_{\mathbf{u} \sim \bar{q}_{t}}\left[\|\mathbf{u}\|^{2}\right] .
$$

which is very similar to the iterations of Algorithm 1 but does not incorporate the AMP Onsager correction terms. The Onsager terms correct for weak dependences in the true posterior distributions of $u_{1}, \ldots, u_{n}$ and of $v_{1}, \ldots, v_{d}$ in high dimensions. These dependences are omitted in the naive mean field approximation, and this is discussed further in [GJM19, FMM21]. The differences between these approaches vanish in the limit of infinite signal-to-noise ratio $s \rightarrow \infty$, but are nonnegligible in any bounded signal-to-noise setting, and become more pronounced for weak signals near the phase transition threshold $s_{*}(\gamma)=\gamma^{-1 / 4}$.

We believe there are two particular appeals of applying AMP over the naive mean field approximation in this specific application: first, in the limit $s \rightarrow \infty$, the sample PCs become increasingly accurate, and there is less to gain from an empirical Bayes approach. It is precisely in settings of weaker signals that empirical Bayes may yield the largest improvements over PCA. Second, even if the model of (2.1) is correctly specified, the connection between the CAVI iterates $\mathbf{f}^{t}, \mathbf{g}^{t}$ and the Gaussian compound decision model is inexact - see Figures 3 and 9-whereas the AMP iterates $\mathbf{f}^{t}, \mathbf{g}^{t}$ are exactly described by the Gaussian models as $n, d \rightarrow \infty$. This provides a stronger justification for applying empirical Bayes procedures based on these Gaussian models to the iterates of AMP. However, we note that EBMF is developed in a more general model of heteroscedastic noise $w_{i j} \sim \mathcal{N}\left(0,1 / \tau_{i j}\right)$, whereas our current derivation and analysis of EB-PCA are limited to a setting of uniform noise variance.

There are a few other distinctions in perspective between EBMF and our work: for the rank- $k$ 
(a)

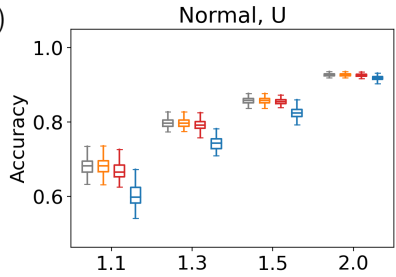

(c)

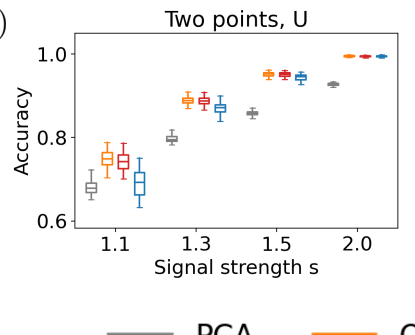

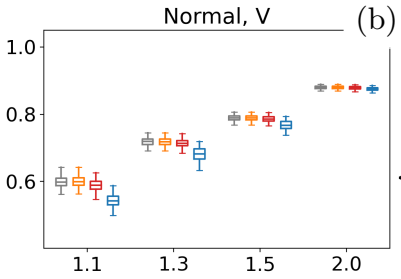

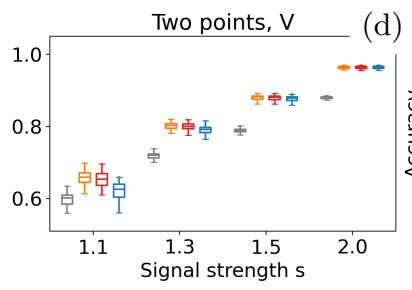

Oracle Bayes AMP

(b)
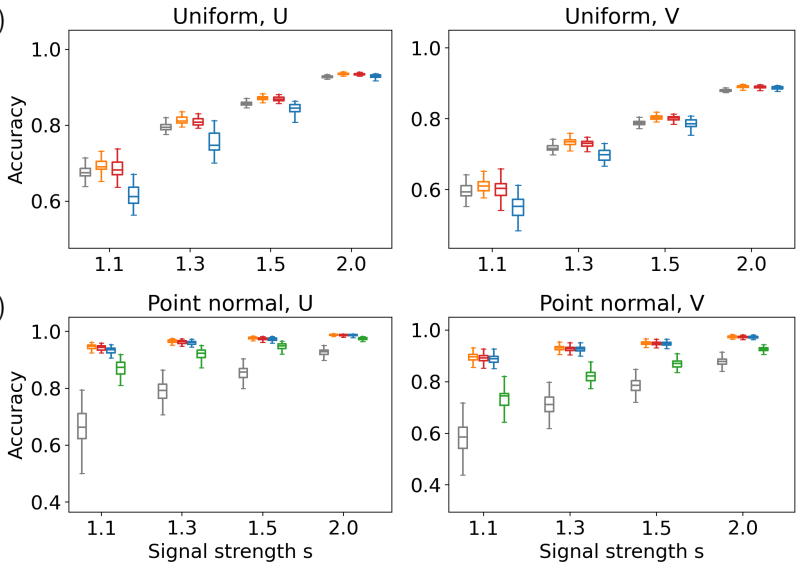

EB-PCA

Figure 2: Estimation accuracy of PCA, oracle Bayes AMP, EB-PCA, and naive mean-field VB, across 50 simulations of a rank-one model with four priors. For (d), comparison with spca is also shown. In all cases, EB-PCA nearly matches the accuracy of the oracle Bayes AMP procedure and improves over mean-field VB. EB-PCA also improves over standard PCA for the non-Gaussian priors $(\mathrm{b}-\mathrm{d})$, and over spca for the sparse prior $(\mathrm{d})$.

model, [WS21] propose a CAVI scheme that iteratively updates each rank-one component separately, and hence does not learn a joint prior for the multivariate distribution of several PCs. For each rank-one component, there is a stronger emphasis in [WS21] on sparsity-inducing priors that are unimodal at 0, connecting the approach more to sparse PCA. Depending on the characteristics of the data at hand, we believe that a multivariate approach of learning a fully nonparametric joint prior for several PCs has the potential of yielding improved accuracy.

\section{$3 \quad$ Simulated examples}

\subsection{Univariate priors}

We compare EB-PCA with several other methods on simulated data from the rank-one model of (1.1), using four different univariate priors: (a) standard Gaussian $\mathcal{N}(0,1)$, (b) Uniform $[-\sqrt{3}, \sqrt{3}]$, (c) Bernoulli $\{+1,-1\}$, and (d) sparse point-normal $0.9 \delta_{0}+0.1 \mathcal{N}(0,10)$. For simplicity, we use the same prior for both $\mathbf{u}$ and $\mathbf{v}$. The two-point and point-normal priors $(\mathrm{c}-\mathrm{d})$ represent simple clustering and sparse-PCA applications.

We compare EB-PCA with standard PCA, the oracle Bayes AMP procedure of [MV21] that knows the true priors, and naive mean-field variational Bayes with priors estimated by NPMLE, corresponding to a version of EBMF in [WS21] with known homoscedastic noise variance. For the sparse point-normal prior (d), we compare also to the spca method of [ZHT06]. Simulation details can be found in Appendix E.

Figure 2 displays the accuracy of these procedures, in terms of the alignments $\langle\hat{\mathbf{u}}, \mathbf{u}\rangle /\|\mathbf{u}\|\|\hat{\mathbf{u}}\|$ and $\langle\hat{\mathbf{v}}, \mathbf{v}\rangle /\|\mathbf{v}\|\|\hat{\mathbf{v}}\|$. These alignments measure the accuracy of the estimated PC directions, and do not account for further improvements of EB-PCA/Bayes-AMP resulting from the shrinkage of $(\mathbf{u}, \mathbf{v})$. The dimensions tested are $(n, d)=(2000,4000)$, so that the estimate of $\mathbf{u} \in \mathbb{R}^{n}$ is more accurate than that of $\mathbf{v} \in \mathbb{R}^{d}$. The phase-transition point for super-critical signal 
strength is $s_{*}(\gamma)=0.84$, and we tested the range of signal strengths $s \in\{1.1,1.3,1.5,2.0\}$.

Under the standard Gaussian prior (a), the posterior mean function in (2.7) for oracle Bayes AMP is linear in each iteration. Thus its iterates remain proportional to the sample PCs, and the asymptotic accuracy of both EB-PCA and Bayes-AMP in the above alignment metric coincide with standard PCA. Thus the Gaussian prior (a) provides a "control" setting in which we hope to match the performance of PCA.

The following observations summarize these comparisons:

- Oracle Bayes AMP. EB-PCA is nearly as accurate as oracle Bayes AMP in all cases, without knowing the true priors. There is a small decrease in accuracy of EB-PCA for very weak signals, due to estimation variance for $s$.

- Standard PCA. In the control setting of the $\mathcal{N}(0,1)$ prior, EB-PCA yields accuracy comparable to PCA. EB-PCA yields improved accuracy in all remaining settings, with this improvement being more substantial for weaker signals and for the two-point and sparse point-normal priors that reflect stronger prior structure.

- SPCA. EB-PCA and mean-field VB both improve significantly over spca for the sparse point-normal prior, with the added advantage of being tuning-parameter free.

- Mean-field VB. EB-PCA improves over mean-field VB in all cases. The improvement is small for the point-normal prior and for larger signal strengths, but is larger in the remaining settings. Mean-field VB seems to yield worse estimation accuracy than standard PCA for the $\mathcal{N}(0,1)$ and continuous uniform priors.

To provide a more detailed comparison of EB-PCA with mean-field VB at a weak signal strength, panels (a) and (b) of Figures 3 and 9 display the entrywise distributions of several iterates for the uniform and two-point priors with $s=1.3$. Overlaid are the convolution densities of the true prior with the estimated levels of Gaussian noise. Panel (c) displays the accuracies across iterations. The overlaid convolution densities in EB-PCA closely match the empirical distributions of the iterates, whereas discrepancies accumulate for mean-field VB. These discrepancies can cause mean-field VB to estimate an increasingly incorrect prior, and to have decreasing accuracy across iterations.

\subsection{Bivariate priors}

We now demonstrate that multivariate EB-PCA, which estimates a joint prior over several PCs, can improve substantially over EB-PCA applied marginally to each PC when there is strong multivariate structure. We consider dimensions $(n, d)=(1000,1000)$ and signal strengths $\left(s_{1}, s_{2}\right)=(4,2)$, for the two bivariate priors presented in Figure 4(a): a discrete three-point prior and a uniform prior on a circle.

Titles in Figure 4 display the estimation error as defined by the subspace distance (see Section 2.5 of [VLG13]) between the column spans of $\hat{\mathbf{U}}$ and $\mathbf{U}$ and also between the spans of the individual PCs. Table 2 reports the average of such estimation errors across 50 simulations. These results indicate that by leveraging the underlying joint structure, multivariate EB-PCA learns a more accurate prior and has lower error both for the estimated two-dimensional subspace and for the individual PCs. The simultaneous estimation of $k$ PCs has the additional benefit of reducing the computation time by a factor of roughly $k$ over the univariate approach. 
(a)
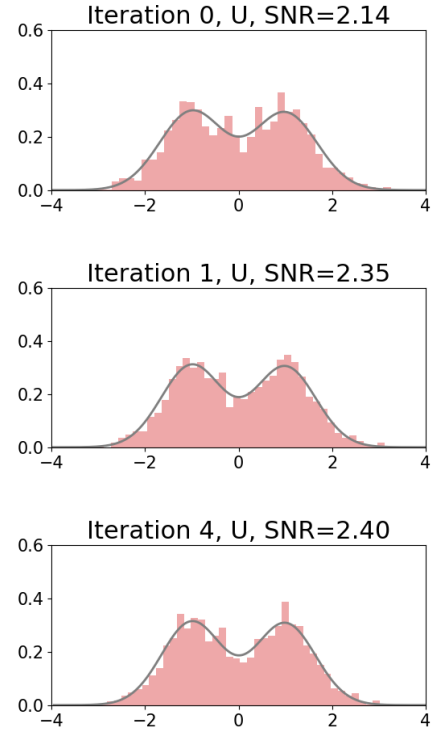

(b)
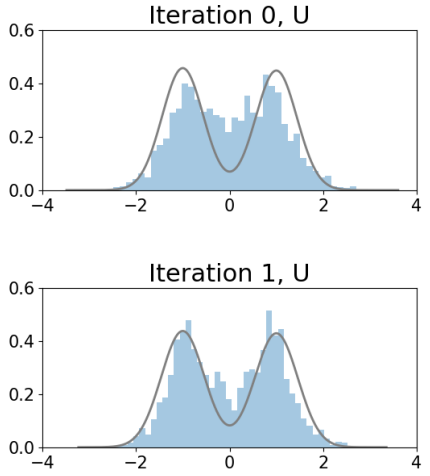

Iteration 4, U

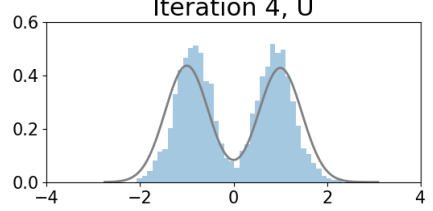

(c)

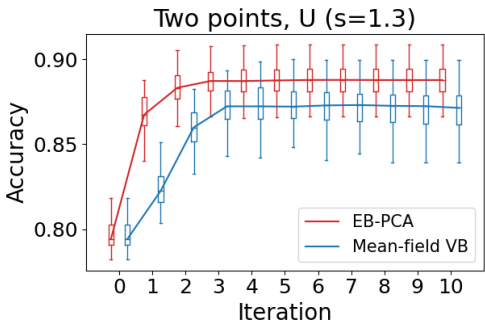

Figure 3: Iterates of EB-PCA versus mean-field VB with Uniform $[-\sqrt{3}, \sqrt{3}]$ priors for $\mathbf{u}$. (a) Distribution of entries of the EB-PCA iterates $\mathbf{f}_{0}, \mathbf{f}_{1}, \mathbf{f}_{4}$. A close agreement is observed with the overlaid convolution densities Uniform $[-\sqrt{3}, \sqrt{3}] * \mathcal{N}\left(0, \bar{\sigma}_{t}^{2} / \bar{\mu}_{t}^{2}\right)$ for $t=0,1$, 4 , where $\bar{\mu}_{t}^{2} / \bar{\sigma}_{t}^{2}$ is the estimated signal-to-noise ratio (SNR) used to perform empirical Bayes denoising. (b) Analogous picture for mean-field VB, with $\bar{\mu}_{t}^{2} / \bar{\sigma}_{t}^{2}$ as estimated in mean-field VB. A discrepancy is observed between the iterates and the convolution densities. (c) Accuracy of $\mathbf{u}_{t}$ across iterations, for EB-PCA versus mean-field VB. Results for $\left(\mathbf{v}, \mathbf{g}_{t}\right)$ are similar and omitted for brevity.

(a)
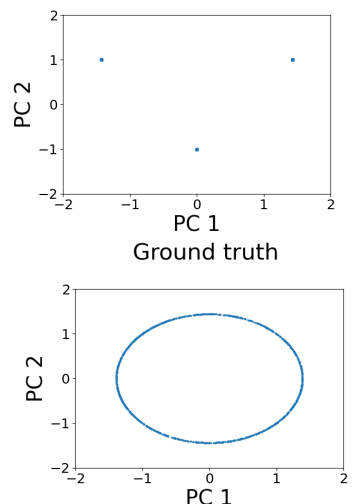

(b)
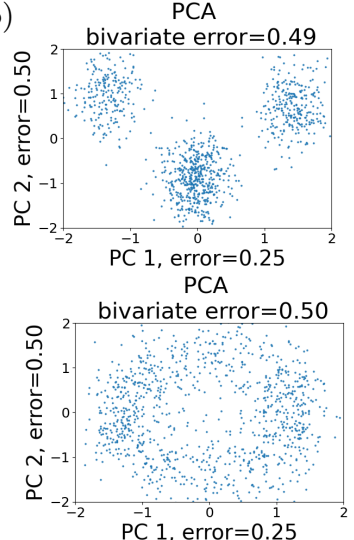

(c)
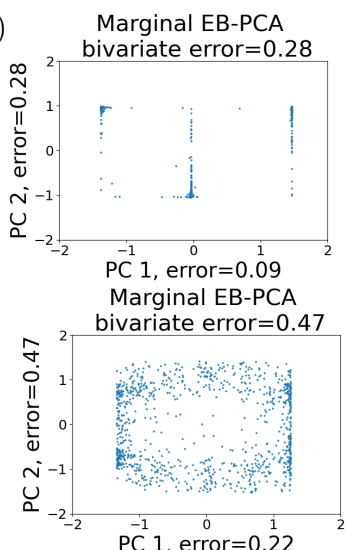

(d)

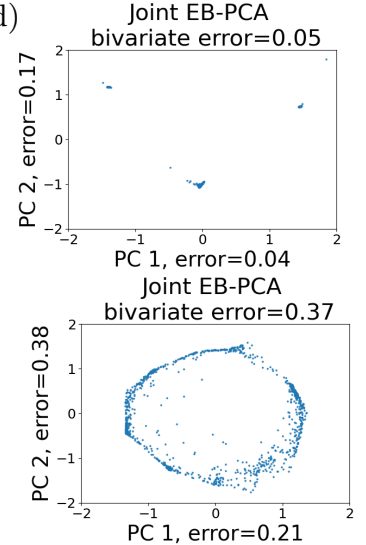

Figure 4: Comparison of standard PCA, EB-PCA applied marginally, and EB-PCA applied jointly for two bivariate priors: a discrete three-point mixture prior (top row) and a uniform prior on a circle (bottom row). Displayed are scatter-plots of the columns of $\mathbf{U}$ for (a) the true PCs, (b) the sample PCs, (c) EB-PCA applied marginally for each PC, and (d) EB-PCA applied jointly to learn the bivariate prior. Estimation errors are displayed in the figure titles. Marginal EB-PCA improves over standard PCA, and joint EB-PCA further improves over marginal EB-PCA. 
Table 1: PC estimation errors on subsampled genotype matrices from 1000 Genomes

\begin{tabular}{c|c|ccccc}
\hline \# SNPs & Error & PC1 & PC2 & PC3 & PC4 & Joint \\
\hline \hline \multirow{2}{*}{100} & PCA & $.35(.039)$ & $.49(.036)$ & $.75(.078)$ & $.83(.059)$ & $.79(.036)$ \\
& EB-PCA & $.20(.058)$ & $.33(.042)$ & $.60(.13)$ & $.62(.12)$ & $.56(.036)$ \\
\hline \multirow{2}{*}{1000} & PCA & $.11(.0073)$ & $.17(.0070)$ & $.31(.024)$ & $.36(.019)$ & $.34(.0091)$ \\
& EB-PCA & $.072(.0099)$ & $.10(.0098)$ & $.17(.042)$ & $.20(.034)$ & $.18(.0055)$ \\
\hline \multirow{2}{*}{10000} & PCA & $.034(.0016)$ & $.051(.0017)$ & $.10(.0089)$ & $.11(.0086)$ & $.11(.0018)$ \\
& EB-PCA & $.028(.0023)$ & $.040(.0032)$ & $.082(.013)$ & $.091(.011)$ & $.081(.0017)$ \\
\hline
\end{tabular}

\section{Applications}

We illustrate EB-PCA on three high-dimensional genetics datasets: genotype data from the 1000 Genomes Project and the third phase of the International HapMap Project (HapMap3) [Int10], and single cell RNA-seq (scRNA-seq) gene expression data on Peripheral Blood Mononuclear Cells (PBMC) from 10X Genomics. Preprocessing procedures and implementation details are provided in Appendix E.

\subsection{Genomes Project genotypes}

PCA is commonly-used to correct for population stratification in genome-wide association studies. As the number of SNPs $n$ often far exceeds the number of individuals $d$, the estimated PCs in $\mathbb{R}^{d}$ suffer minimally from high-dimensional noise for the leading PCs. This provides a ground truth by which we may quantitatively compare estimation accuracy on subsampled data.

Implementing this experiment for genotype data from the 1000 Genomes Project, we extracted 100,000 common SNPs for 2504 individuals, from which we computed the ground truth PCs. We then estimated PCs on subsamples of 100, 1000, or 10,000 randomly selected SNPs using both EBPCA and PCA. Figure 8 plots the singular values for a typical subsample of 1000 SNPs, in which 4 clear outlier values are apparent. Thus we chose to estimate the leading 4 PCs using EB-PCA.

Table 1 compares errors across 50 random subsamples of each size, where the error is the subspace distance (as previously used in Section 3.2) against the ground truth PCs. The results show a clear improvement of EB-PCA over PCA, with slightly larger improvement for the lower PCs having smaller singular values.

A visual comparison for 1000 subsampled SNPs was presented previously in Figure 1 . The EB-PCA estimates are closer to the ground truth than the sample PCs, and better separate the subjects by ethnicity. For example, the Caucasian, African, and East Asian populations are mixed in the two-dimensional plot of the 3rd vs. 4th sample PC, whereas they are separated in the EBPCA estimates and also in the ground truth. The information used by EB-PCA for performing this separation in PCs 3 and 4 comes from learning a joint prior with PCs 1 and 2, where these populations have clear separation.

\subsection{HapMap3 genotypes}

We performed a similar experiment on genotype data from HapMap3. For the 1397 individuals in HapMap3, we computed ground truth PCs from 142,185 common SNPs. Based on the singular value distribution in Figure 8 on a subsample of 5000 SNPs, we chose to estimate the leading 4 PCs using EB-PCA. 
(a)

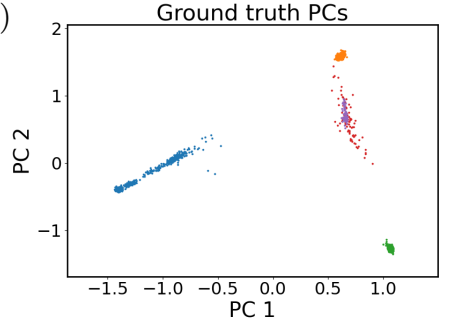

Ground truth PCs

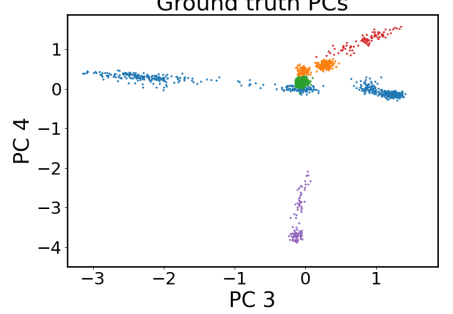

- African - Caucasian (b)
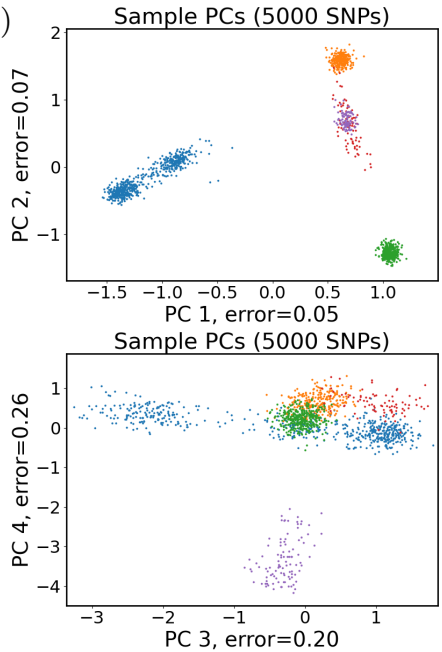

- East-Asian (c)

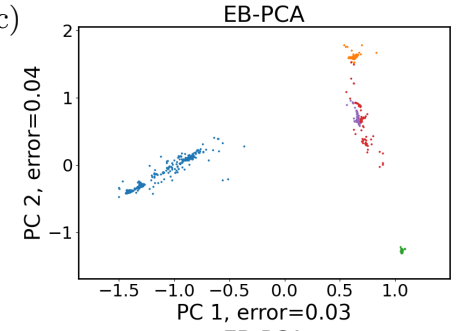

EB-PCA

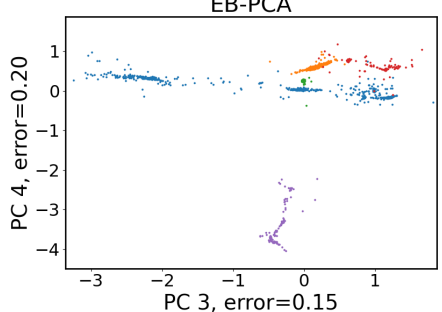

- South Asian

Figure 5: Illustration of EB-PCA on genotype data from HapMap3, similar to Figure 1. (a) Ground truth PCs, with points colored by the individuals' ethnicity. (b) Sample PCs computed on a subsample of 5000 SNPs. (c) EB-PCA estimates of the four PCs, using the same subsample as in panel (b).

Table 3 compares the errors of EB-PCA and PCA across 50 subsampled data sets of 1000 , 5000, and 10,000 SNPs, and Figure 5 depicts results for 5000 SNPs. We again observe a consistent decrease in estimation error for EB-PCA, which is larger for the weaker PCs.

\subsection{0x Genomics PBMC single-cell RNA-seq}

PCA is often the first step in single-cell gene expression data analysis pipelines such as Seurat and scanpy, to capture the signatures of cell identity. Nonlinear dimensionality reduction methods are often then applied with the estimated PCs as input, to perform cell clustering and to infer cell types.

We illustrate an application of EB-PCA on scRNA-seq data of $d=2626$ Peripheral Blood Mononuclear Cells (PBMCs) with $n=13,711$ gene expressions, from 10X Genomics. This data is

(a)

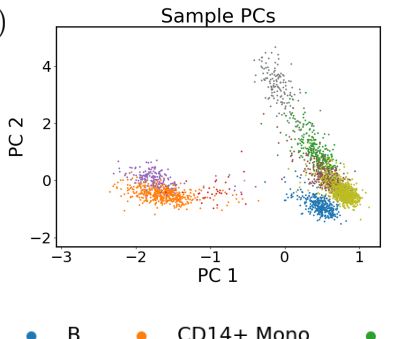

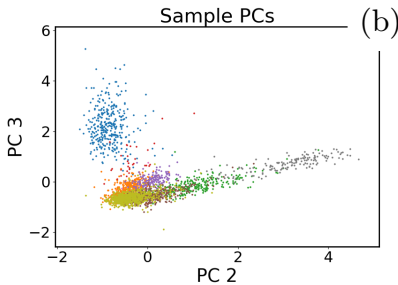

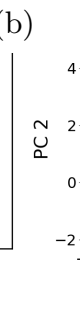

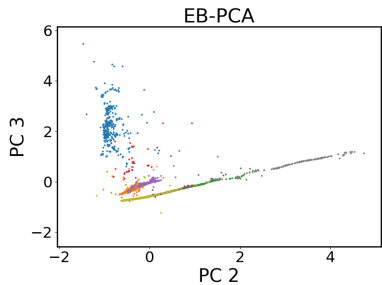

Mk

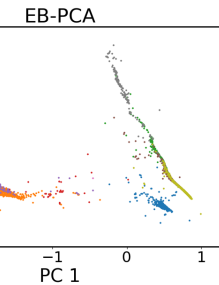

Memory CD4 T

Figure 6: Estimated PCs using (a) standard PCA and (b) EB-PCA, for PBMC single-cell RNAseq gene expression data, with points colored by cell type. The EB-PCA estimates exhibit reduced estimation noise and clearer separation of cells by cell type. 
more representative of typical applications of EB-PCA, in which there are insufficient samples to provide a known ground truth. Therefore, we applied EB-PCA without subsampling. The singular values are shown in Figure 8, and 3 large outlier values are apparent. The corresponding leading 3 PCs are depicted as two scatterplots in Figure 6(a). Qualitatively, these scatterplots exhibit estimation noise that resembles the noise previously observed in the subsampled 1000 Genomes and HapMap3 genotype matrices, suggesting that the noise may be a consequence of high dimensionality.

Figure 6(b) displays the estimated PCs using EB-PCA. Although there is not a basis for quantitative comparison with PCA in this example, we believe that the EB-PCA results may be more accurate for an underlying ground truth. Qualitatively, the estimation noise exhibited in Figure 6(a) has been reduced. There is a clearer separation between different cell types. For example, Naïve CD4 T cells are separated from CD14+ Monocytes in the plot of the 2nd vs. 3rd PCs estimated by EB-PCA, whereas these overlap in the plot of the sample PCs.

\section{Theoretical guarantees}

In this section, we summarize theoretical guarantees for EB-PCA. In the context of the signal-plusnoise model (2.1), our results show that EB-PCA asymptotically achieves the same (first-order) estimation accuracy as the oracle Bayes AMP procedure. Consequently, the asymptotic estimation error provably decreases across iterations, and can approach the Bayes-optimal error as the number of iterations $t \rightarrow \infty$ under general conditions for the true priors.

The strategy of proof is to first establish asymptotic consistency of the NPMLEs for the prior distributions, and then, through an inductive comparison argument, show that the iterates of EBPCA are characterized by the same state evolution as oracle Bayes AMP. We note that prior work on asymptotic consistency for the NPMLE assumes that a compound decision model (2.6) with i.i.d. Gaussian errors holds exactly, whereas such an error model holds only approximately for the sample PCs and AMP iterates. Our arguments show that this approximation is sufficient for asymptotic consistency.

\subsection{Assumptions}

Consider the rank- $k$ model of (2.1). We assume the empirical distributions of rows of $\mathbf{U} \in \mathbb{R}^{n \times k}$ and $\mathbf{V} \in \mathbb{R}^{d \times k}$ converge in Wasserstein-2 distance to fixed distributions $\bar{\pi}_{*}, \pi_{*}$ on $\mathbb{R}^{k}$, as $n, d \rightarrow \infty$. This means that for any continuous function $\psi: \mathbb{R}^{k} \rightarrow \mathbb{R}$ with $\mathbb{E}_{U \sim \bar{\pi}_{*}}\left[\psi(U)^{2}\right]<\infty$, we have $\lim _{n \rightarrow \infty} n^{-1} \sum_{i=1}^{n} \psi\left(u_{i 1}, \ldots, u_{i k}\right)=\mathbb{E}_{U \sim \bar{\pi}_{*}}[\psi(U)]$, and similarly for $\mathbf{V}$. We denote this convergence both as $\mathbf{U} \stackrel{W_{2}}{\rightarrow} \bar{\pi}_{*}$ and as $\mathbf{U} \stackrel{W_{2}}{\rightarrow} U$ for a random vector $U \sim \bar{\pi}_{*}$. Our model assumptions are then summarized as follows.

Assumption 5.1. $\mathcal{P}$ is a family of probability distributions on $\mathbb{R}^{k}$ having finite second moment, and $n, d \rightarrow \infty$ such that

(a) $\mathbf{W} \in \mathbb{R}^{n \times d}$ has entries $w_{i j} \stackrel{i i d}{\sim} \mathcal{N}(0,1 / n)$.

(b) $k, s_{1}, \ldots, s_{k}$, and $\gamma \equiv d / n$ remain constant, where $s_{1}>\ldots>s_{k}>s_{*}(\gamma) \equiv \gamma^{-1 / 4}$.

(c) $\mathbf{U} \stackrel{W_{2}}{\rightarrow} \bar{\pi}_{*}$ and $\mathbf{V} \stackrel{W_{2}}{\rightarrow} \pi_{*}$ for two distributions $\bar{\pi}_{*}, \pi_{*} \in \mathcal{P}$ that satisfy the normalizations, for all $1 \leq i \neq j \leq k$

$$
\mathbb{E}_{U \sim \bar{\pi}_{*}}\left[U_{i}^{2}\right]=1, \quad \mathbb{E}_{U \sim \bar{\pi}_{*}}\left[U_{i} U_{j}\right]=0, \quad \mathbb{E}_{V \sim \pi_{*}}\left[V_{i}^{2}\right]=1, \quad \mathbb{E}_{V \sim \pi_{*}}\left[V_{i} V_{j}\right]=0
$$

(d) For any non-singular $M_{*} \in \mathbb{R}^{k \times k}$, symmetric positive-definite $\Sigma_{*} \in \mathbb{R}^{k \times k}$, and $\pi_{*} \in \mathcal{P}$, there is a weakly open neighborhood $O$ of $\pi_{*}$ such that $\theta\left(x \mid M_{*}, \Sigma_{*}, \pi\right)$ is Lipschitz in $x$ uniformly over $\pi \in O$. 
Part (a) makes a Gaussian assumption for the noise, and part (b) assumes for simplicity that the signal values $s_{1}, \ldots, s_{k}$ are distinct and super-critical.

Part (c) ensures the normalizations in (2.3) and (2.4). This can hold both when $\mathbf{U} \in \mathbb{R}^{n \times k}$ and $\mathbf{V} \in \mathbb{R}^{d \times k}$ are deterministic matrices whose columns are the (exactly) orthogonal true PCs, as well as almost surely in a Bayesian setting when $\mathbf{U}$ and $\mathbf{V}$ are random with independent rows generated from $\bar{\pi}_{*}$ and $\pi_{*}$. We will assume $\mathbf{U}$ and $\mathbf{V}$ are deterministic, i.e. our results apply conditional on $(\mathbf{U}, \mathbf{V})$ in the Bayesian setting.

Part (d) assumes a Lipschitz property for the posterior mean functions, as is common in analyses of AMP. This places a small restriction on the prior class $\mathcal{P}$; for example, the assumption holds if $\mathcal{P}$ is the class of all priors supported on a compact domain of $\mathbb{R}^{k}$.

We analyze EB-PCA in a slightly idealized setting where the noise variance $1 / n$ in part (a) is known, rather than estimated as in Remark 2.1, and where the NPMLE in Lines 5 and 9 of Algorithm 1 are computed exactly in each iteration. Our results may be extended to incorporate a consistent estimate of the noise variance and an approximate NPMLE computed on a sufficiently fine discretization of the support, by a standard comparison argument with this idealized settingwe omit the details of such an argument for brevity.

\subsection{Limiting risk for the initial empirical Bayes estimates}

In the compound decision model $\Theta \sim \pi$ and $X \mid \Theta \sim \mathcal{N}(M \cdot \Theta, \Sigma)$, we denote the squared error Bayes risk for estimating $\Theta$ based on $X$ as

$$
\operatorname{mmse}(\pi \mid M, \Sigma)=\mathbb{E}\left[\|\Theta-\mathbb{E}[\Theta \mid X]\|_{2}^{2}\right] .
$$

The entrywise Gaussian approximation in (2.13) for the sample PCs is formalized as the following proposition. Lemma C.1 of [MV21] proves a similar result for the symmetric spiked model.

Proposition 5.2. Let $\bar{M}_{*}, M_{*}, \bar{\Sigma}_{*}, \Sigma_{*} \in \mathbb{R}^{k \times k}$ be defined by (2.11) and (2.12). Under Assumption 5.1 , almost surely $(\mathbf{U}, \mathbf{F}) \stackrel{W_{2}}{\rightarrow}(U, F)$ and $(\mathbf{V}, \mathbf{G}) \stackrel{W_{2}}{\rightarrow}(V, G)$ where $U \sim \bar{\pi}_{*}, F \mid U \sim \mathcal{N}\left(\bar{M}_{*} \cdot U, \bar{\Sigma}_{*}\right)$, $V \sim \pi_{*}$, and $G \mid V \sim \mathcal{N}\left(M_{*} \cdot V, \Sigma_{*}\right)$.

Combined with an asymptotic consistency result for the NPMLE in approximate compound decision models, shown in Lemma B.2 and Corollary B.3, this yields the following asymptotic squared-error risks for the initial empirical Bayes estimates of $\mathbf{U}$ and $\mathbf{V}$.

Corollary 5.3. Let $\hat{\mathbf{U}}$ and $\hat{\mathbf{V}}$ be the initial empirical Bayes estimators in (2.15) and (2.16). Let $\bar{M}_{*}, M_{*}, \bar{\Sigma}_{*}, \Sigma_{*} \in \mathbb{R}^{k \times k}$ be defined by (2.11) and (2.12). Under Assumption 5.1, almost surely

$$
n^{-1}\|\hat{\mathbf{U}}-\mathbf{U}\|_{F}^{2} \rightarrow \operatorname{mmse}\left(\bar{\pi}_{*} \mid \bar{M}_{*}, \bar{\Sigma}_{*}\right), \quad d^{-1}\|\hat{\mathbf{V}}-\mathbf{V}\|_{F}^{2} \rightarrow \operatorname{mmse}\left(\pi_{*} \mid M_{*}, \Sigma_{*}\right) .
$$

Thus the asymptotic squared-error risk for $\hat{\mathbf{U}}$ is the Bayes risk for estimating $\Theta \sim \bar{\pi}_{*}$ based on $X \mid \Theta \sim \mathcal{N}\left(\bar{M}_{*} \cdot \Theta, \bar{\Sigma}_{*}\right)$, and similarly for $\hat{\mathbf{V}}$. In contrast, the analogous risks for the naive sample PCs $\mathbf{F}$ and $\mathbf{G}$, or more generally of the best shrinkage estimators obtained by rescaling their columns, correspond to the Bayes risks of the best linear estimators of $\Theta$ in these compound decision problems. These linear risks may be substantially larger if the priors $\bar{\pi}_{*}$ and $\pi_{*}$ are far from the standard Gaussian law. 


\subsection{Limiting risk and Bayes optimality of EB-PCA}

For the full EB-PCA method, the following verifies that the NPMLEs $\bar{\pi}_{t}, \pi_{t}$ remain consistent for $\bar{\pi}_{*}, \pi_{*}$ across iterations. Consequently, the EB-PCA iterates satisfy the same Gaussian approximations (2.18) and are tracked by the same state evolution (2.19) as the oracle Bayes AMP algorithm.

Theorem 5.4. Suppose Assumption 5.1 holds. Then almost surely for each fixed iteration $t \in$ $\{0, \ldots, T\}$ of Algorithm 1, $\pi_{t}$ converges weakly to $\pi_{*}$ and $\bar{\pi}_{t}$ converges weakly to $\bar{\pi}_{*}$. Furthermore,

$$
\left(\mathbf{U}, \mathbf{F}^{t}\right) \stackrel{W_{2}}{\rightarrow}\left(U, F_{t}\right) \quad \text { and } \quad\left(\mathbf{V}, \mathbf{G}^{t}\right) \stackrel{W_{2}}{\rightarrow}\left(V, G_{t}\right)
$$

where $U \sim \bar{\pi}_{*}, F_{t} \mid U \sim \mathcal{N}\left(\bar{M}_{*, t} \cdot U, \bar{\Sigma}_{*, t}\right), V \sim \pi_{*}$, and $G_{t} \mid V \sim \mathcal{N}\left(M_{*, t} \cdot V, \Sigma_{*, t}\right)$. The matrices $\bar{M}_{*, t}, \bar{\Sigma}_{*, t}, M_{*, t}, \Sigma_{*, t}$ are defined iteratively by the state evolution in (2.19).

As a corollary, the asymptotic squared-error risk for each EB-PCA iterate is the same as that achieved by the oracle Bayes AMP algorithm with known priors.

Corollary 5.5. Let $\bar{M}_{*, t}, \bar{\Sigma}_{*, t}, M_{*, t}, \Sigma_{*, t}$ be defined iteratively by the state evolution in (2.19). Under Assumption 5.1, almost surely for each fixed iteration $t \in\{0, \ldots, T\}$,

$$
\frac{1}{n}\left\|\mathbf{U}^{t}-\mathbf{U}\right\|_{F}^{2} \rightarrow \operatorname{mmse}\left(\bar{\pi}_{*} \mid \bar{M}_{*, t}, \bar{\Sigma}_{*, t}\right), \quad \frac{1}{d}\left\|\mathbf{V}^{t}-\mathbf{V}\right\|_{F}^{2} \rightarrow \operatorname{mmse}\left(\pi_{*} \mid M_{*, t}, \Sigma_{*, t}\right) .
$$

To study the decrease of these errors across iterations, we follow [Mio17] and introduce the positive-definite state matrices

$$
\bar{Q}_{*, t}=\frac{1}{\gamma} S^{-1 / 2} \bar{M}_{*, t}^{\top} \bar{\Sigma}_{*, t}^{-1} \bar{M}_{*, t} S^{-1 / 2}, \quad Q_{*, t}=S^{-1 / 2} M_{*, t}^{\top} \Sigma_{*, t}^{-1} M_{*, t} S^{-1 / 2} .
$$

These are matrix-valued measures of the signal-to-noise ratios in the compound decision models associated to $\mathbf{F}^{t}$ and $\mathbf{G}^{t}$ in each iteration. For the standardized compound decision model

$$
\Theta \sim \pi, \quad X \mid \Theta \sim \mathcal{N}\left(\Theta, Q^{-1}\right)
$$

(with $M=\mathrm{Id}$ ) parametrized by $\pi$ and $Q$, define the map

$$
F_{\pi}(Q)=\mathbb{E}\left[\mathbb{E}[\Theta \mid X] \cdot \mathbb{E}[\Theta \mid X]^{\top}\right] .
$$

We verify in Appendix D that the state evolution in (2.19) is equivalently expressed as

$$
\bar{Q}_{*, t}=F_{S^{1 / 2} \pi_{*}}\left(Q_{*, t}\right), \quad Q_{*, t+1}=F_{S^{1 / 2} \bar{\pi}_{*}}\left(\gamma \cdot \bar{Q}_{*, t}\right),
$$

where $S^{1 / 2} \pi$ is the distribution of $S^{1 / 2} \cdot \Theta$ when $\Theta \sim \pi$. The following shows that the state evolution converges to a fixed point of these maps, the squared-error risks of the EB-PCA iterates improve over the initial empirical Bayes estimates, and these risks decrease monotonically over iterations.

Proposition 5.6. Under Assumption 5.1, for each $t=0,1,2, \ldots$

(a) $\bar{Q}_{*, t+1} \succeq \bar{Q}_{*, t}$ and $Q_{*, t+1} \succeq Q_{*, t}$. Furthermore, as $t \rightarrow \infty$, these matrices converge to a fixed point of

$$
\bar{Q}=F_{S^{1 / 2} \pi_{*}}(Q), \quad Q=F_{S^{1 / 2} \bar{\pi}_{*}}(\gamma \cdot \bar{Q}) .
$$

(b) Let $\operatorname{mmse}\left(\bar{\pi}_{*} \mid \bar{M}_{*}, \bar{\Sigma}_{*}\right)$ and $\operatorname{mmse}\left(\pi_{*} \mid M_{*}, \Sigma_{*}\right)$ be the risks of the initial empirical Bayes estimates in Corollary 5.3. Then the asymptotic risks in (5.1) satisfy

$$
\begin{aligned}
& \operatorname{mmse}\left(\bar{\pi}_{*} \mid \bar{M}_{*, t+1}, \bar{\Sigma}_{*, t+1}\right) \leq \operatorname{mmse}\left(\bar{\pi}_{*} \mid \bar{M}_{*, t}, \bar{\Sigma}_{*, t}\right) \leq \operatorname{mmse}\left(\bar{\pi}_{*} \mid \bar{M}_{*}, \bar{\Sigma}_{*}\right), \\
& \operatorname{mmse}\left(\pi_{*} \mid M_{*, t+1}, \Sigma_{*, t+1}\right) \leq \operatorname{mmse}\left(\pi_{*} \mid M_{*, t}, \Sigma_{*, t}\right) \leq \operatorname{mmse}\left(\pi_{*} \mid M_{*}, \Sigma_{*}\right) .
\end{aligned}
$$


Finally, suppose $\bar{\pi}_{*}, \pi_{*}$ are such that (5.3) has a unique fixed point $(\bar{Q}, Q)$. In the Bayesian setting where rows of $\mathbf{U}$ and $\mathbf{V}$ are random and i.i.d., Proposition 3 of [Mio17] shows that this fixed point characterizes the Bayes-optimal squared-error risk for estimating $\mathbf{U} S \mathbf{V}^{\top}$ :

$$
\lim _{n, d \rightarrow \infty}\left(\inf _{\hat{\mathbf{X}}} \frac{1}{n d} \mathbb{E}\left[\left\|\hat{\mathbf{X}}(\mathbf{Y})-\mathbf{U} S \mathbf{V}^{\top}\right\|_{F}^{2}\right]\right)=\operatorname{Tr} \mathbb{E}_{(U, V) \sim \bar{\pi}_{*} \times \pi_{*}}\left[U U^{\top} S V V^{\top} S\right]-\operatorname{Tr} \bar{Q} Q,
$$

where the infimum is over all (measurable) estimators $\hat{\mathbf{X}}(\mathbf{Y})$ and achieved at the Bayes estimator $\mathbb{E}\left[\mathbf{U} S \mathbf{V}^{\top} \mid \mathbf{Y}\right]$. Exact computation of this Bayes estimator may be intractable. The below verifies that the asymptotic error of EB-PCA (and hence also of the oracle Bayes AMP algorithm) approaches this Bayes-optimal error as $t \rightarrow \infty$. This type of result has been stated for rank $k=1$ in $\left[\mathrm{BDM}^{+} 16, \mathrm{MV} 21\right]$.

Proposition 5.7. Under Assumption 5.1, suppose the fixed point $(\bar{Q}, Q)$ of (5.3) is unique. For any fixed $t \geq 1$, let $\left(\mathbf{U}^{t}, \mathbf{V}^{t}\right)$ be the EB-PCA estimates of $(\mathbf{U}, \mathbf{V})$ in iteration $t$ of Algorithm 1, and let $\hat{S}=\operatorname{diag}\left(\hat{s}_{1}, \ldots, \hat{s}_{k}\right)$ be the estimate of $S$ from (2.14). Then almost surely as $n, d \rightarrow \infty$,

$$
\frac{1}{n d}\left\|\mathbf{U}^{t} \hat{S}\left(\mathbf{V}^{t}\right)^{\top} \quad-\quad \mathbf{U} S \mathbf{V}^{\top}\right\|_{F}^{2} \quad \rightarrow \quad \operatorname{Tr} \mathbb{E}_{(U, V) \sim \bar{\pi}_{*} \times \pi_{*}}\left[U U^{\top} S V V^{\top} S\right]-\operatorname{Tr} \bar{Q} Q \quad-o_{t}(1)
$$

where $o_{t}(1)$ is a deterministic quantity satisfying $o_{t}(1) \rightarrow 0$ as $t \rightarrow \infty$.

For rank $k=1$, we refer readers to $\left[\mathrm{BDM}^{+} 16, \mathrm{MV} 21, \mathrm{LM} 19\right]$ for examples of priors $\bar{\pi}_{*}, \pi_{*}$ for which uniqueness of the fixed point to (5.3) does and does not hold. It has been conjectured that in examples where this fixed point is not unique, the asymptotic risk corresponding to the fixed point that is reached by EB-PCA/oracle-Bayes AMP is the smallest Bayes risk that is attainable by any estimator in polynomial time [LKZ15b, BDM+16, LM19, AFUZ19].

\section{Conclusion}

We have described an EB-PCA procedure for performing PCA in high dimensions, which couples classical empirical Bayes ideas with high-dimensional asymptotic theory. In applications where the joint distribution of PCs has non-Gaussian structure, EB-PCA can improve estimation accuracy by obtaining a nonparametric estimate of this structure.

EB-PCA is an example of a more general paradigm of carrying out "TAP-corrected" variational Bayesian inference in high dimensions using an empirical Bayes approach. The high dimensionality of the latent variable space becomes a blessing in such problems, enabling the estimation of complex and nonparametric prior distributions for these latent variables using empirical Bayes ideas. This general paradigm may potentially be implemented with other inference algorithms and extended to other inference problems.

We conclude with a discussion of a direction for future work. The quantitative form of EBPCA - and of AMP-based procedures more generally - is derived assuming that the noise matrix W has independent entries with common variance. (To keep the proofs simple, we have also assumed that these entries are Gaussian, but it is known that the Gaussian assumption can be substantially weakened: universality results of this type have been shown for the sample PCs in [BDW21, BDWW20], and for the AMP state evolution in [BLM15, CL21].)

It is an open question to extend the procedure to settings where $\mathbf{W}$ has correlation structure, which is commonly reflected in data by an overdispersed singular value distribution. One such setting that is partially understood is that of bi-rotationally invariant matrices $\mathbf{W}$ that satisfy the equality in law $\mathbf{W} \stackrel{L}{=} \mathbf{O}^{\top} \mathbf{W} \mathbf{Q}$ for any orthogonal matrices $\mathbf{O} \in \mathbb{R}^{n \times n}$ and $\mathbf{Q} \in \mathbb{R}^{d \times d}$. This reflects 
an assumption that W may have an arbitrary distribution of singular values but "generic" singular vectors. The asymptotic behavior of sample PCs in this setting was studied by [BGN12], who provided the quantitative forms for the limits (2.10). In the rank-one case, it was shown also in [Fan20] that the Onsager correction in (2.17) and AMP state evolution (2.19) are to be replaced by certain series expressions defined by the free cumulants of $\mathbf{W}$, which may be estimated from the singular value distribution of $\mathbf{Y}$.

Other models for $\mathbf{W}$ - for example general covariance models $\mathbf{W}=\mathbf{Z B}^{1 / 2}$ [BY12, BDWW20] or separable covariance models $\mathbf{W}=\mathbf{A}^{1 / 2} \mathbf{Z B}^{1 / 2}$ [Yan19, DY21] where $\mathbf{Z} \in \mathbb{R}^{n \times d}$ has i.i.d. entriesmay also be studied. Developing variational Bayesian procedures that are both asymptotically exact and computationally efficient in these and related models is an interesting direction for future work, and we believe it is likely that such developments may improve and robustify the EB-PCA procedure, when they become available.

\section{Acknowledgments}

We thank Yixuan Ye, Chao Zhou and Hongyu Zhao for their help in collecting and interpreting data, and Yihong Wu for helpful discussions about the Kiefer-Wolfowitz NPMLE and ideas in the early stages of the theoretical analyses. This research was supported in part by NSF Grant DMS-1916198. 


\section{A Proof Preliminaries}

Notations and conventions. We reserve bold-face letters for vectors and matrices involving the increasing dimensions $n$ and $d . \mathbf{u} \otimes \mathbf{v}=\mathbf{u v}^{\top}$ is the outer product, and $\mathbf{u}^{\otimes 2}=\mathbf{u} \otimes \mathbf{u}$. For a function $f: \mathbb{R}^{p} \rightarrow \mathbb{R}^{q}, \mathrm{~d} f \in \mathbb{R}^{q \times p}$ is its Jacobian matrix. When $q=1, \mathrm{~d} f \in \mathbb{R}^{1 \times p}$ is the gradient as a row vector, $\mathrm{d}^{2} f \in \mathbb{R}^{p \times p}$ is the Hessian, and $\mathrm{d}^{j} f \in \mathbb{R}^{p \times \ldots \times p}$ is the tensor of $j^{\text {th }}$ partial derivatives.

$\|\cdot\|$ is the Euclidean norm for vectors and Euclidean operator norm for matrices and tensors. $\|\cdot\|_{F}$ is the Frobenius norm for matrices. $\mathbb{B}^{k}(B)=\left\{x \in \mathbb{R}^{k}:\|x\| \leq B\right\}$ is the closed ball of radius $B$. For positive-definite $\Sigma, \phi_{\Sigma}(x)$ is the Lebesgue density function of the distribution $\mathcal{N}(0, \Sigma)$. $f_{(M \cdot \pi) * \mathcal{N}(0, \Sigma)}(x)$ is the marginal density of $X$ in the model $\Theta \sim \pi$ and $X \mid \Theta \sim \mathcal{N}(M \cdot \Theta, \Sigma)$.

\section{A.1 Wasserstein convergence and pseudo-Lipschitz functions}

Definition A.1 (Wasserstein convergence). Let $\pi_{n}, \pi$ be probability measures on $\mathbb{R}^{k}$ with finite second moment. Then $\pi_{n}$ converges to $\pi$ in the Wasserstein- 2 distance as $n \rightarrow \infty$ if, for any $C>0$ and continuous function $f: \mathbb{R}^{k} \rightarrow \mathbb{R}$ satisfying

$$
f(u) \leq C\left(1+\|u\|^{2}\right) \quad \text { for all } u \in \mathbb{R}^{k},
$$

we have $\mathbb{E}_{U \sim \pi_{n}}[f(U)] \rightarrow \mathbb{E}_{U \sim \pi}[f(U)]$.

For a matrix $\mathbf{U} \in \mathbb{R}^{n \times k}$, we write $\mathbf{U} \stackrel{W_{2}}{\rightarrow} \pi$ if the empirical distribution of rows of $\mathbf{U}$ converges in Wasserstein-2 distance to $\pi$. We denote this also as $\mathbf{U} \stackrel{W_{2}}{\rightarrow} U$, for $U \sim \pi$.

Definition A.2 (Pseudo-Lipschitz functions). A function $\psi: \mathbb{R}^{k} \rightarrow \mathbb{R}$ is 2-pseudo-Lipschitz if there exists a constant $L>0$ such that for all $u, u^{\prime} \in \mathbb{R}^{k}$,

$$
\left|\psi(u)-\psi\left(u^{\prime}\right)\right| \leq L\left(1+\|u\|+\left\|u^{\prime}\right\|\right)\left\|u-u^{\prime}\right\| .
$$

We write $\psi \in \mathrm{PL}(2)$.

Note that any $\psi \in \mathrm{PL}(2)$ satisfies $\psi(u) \leq \psi(0)+L(1+\|u\|)\|u\| \leq C(1+\|u\|)^{2}$, so if $\mathbf{U} \stackrel{W_{2}}{\rightarrow} U$ and $\mathbf{U}$ has rows $u_{1}, \ldots, u_{n}$, then

$$
n^{-1} \sum_{i=1}^{n} \psi\left(u_{i}\right) \rightarrow \mathbb{E}[\psi(U)]
$$

\section{A.2 Data normalization}

Consider the model

$$
\mathbf{Y}_{\mathrm{obs}}=\frac{1}{n} \cdot \mathbf{U} S \mathbf{V}^{\top}+\mathbf{W}
$$

where $\mathbf{U} S \mathbf{V}^{\top}$ is any matrix of $\operatorname{rank} k$, and $w_{i j} \stackrel{i i d}{\sim} \mathcal{N}\left(0, \tau^{2} / n\right)$. We show that the estimate $\hat{\tau}^{2}$ in (2.2) of the Main Text is consistent for $\tau^{2}$, for fixed $k$ as $n, d \rightarrow \infty$ (at any rates, and not necessarily proportionally).

Let $\lambda_{1}\left(\mathbf{Y}_{\mathrm{obs}}\right) \geq \ldots \geq \lambda_{d}\left(\mathbf{Y}_{\mathrm{obs}}\right)$ be the singular values of $\mathbf{Y}_{\mathrm{obs}}$, where the last $d-n$ are 0 if $d>n$. Then

$$
\hat{\tau}^{2}=\frac{1}{d} \sum_{i=k+1}^{d} \lambda_{i}\left(\mathbf{Y}_{\mathrm{obs}}\right)^{2} .
$$


Let $\lambda_{1}(\mathbf{W}) \geq \ldots \geq \lambda_{d}(\mathbf{W})$ be the singular values of $\mathbf{W}$. By Weyl's inequality,

$$
\lambda_{i}\left(\mathbf{Y}_{\mathrm{obs}}\right) \leq \lambda_{i-k}(\mathbf{W}) \text { for all } i \geq k+1, \quad \lambda_{i}\left(\mathbf{Y}_{\mathrm{obs}}\right) \geq \lambda_{i+k}(\mathbf{W}) \text { for all } i \leq d-k .
$$

Applying this to the above form of $\hat{\tau}^{2}$,

$$
\frac{1}{d}\|\mathbf{W}\|_{F}^{2}-\frac{1}{d} \sum_{i=1}^{2 k} \lambda_{i}(\mathbf{W})^{2}=\frac{1}{d} \sum_{i=2 k+1}^{d} \lambda_{i}(\mathbf{W})^{2} \leq \hat{\tau}^{2} \leq \frac{1}{d} \sum_{i=1}^{d-k} \lambda_{i}(\mathbf{W})^{2} \leq \frac{1}{d}\|\mathbf{W}\|_{F}^{2} .
$$

Since $w_{i j} \stackrel{\text { iid }}{\sim} \mathcal{N}\left(0, \tau^{2} / n\right)$, we have $d^{-1}\|\mathbf{W}\|_{F}^{2}=d^{-1} \sum_{i, j} w_{i j}^{2} \rightarrow \tau^{2}$ a.s. as $n, d \rightarrow \infty$ by the law of large numbers. We have also $\lambda_{i}(\mathbf{W}) \leq \lambda_{1}(\mathbf{W}) \leq C(1+\sqrt{d / n})$ a.s. for an absolute constant $C>0$ and all large $n$ and $d$, see e.g. Theorem 4.4 .5 of [Ver18]. So $d^{-1} \sum_{i=1}^{2 k} \lambda_{i}(\mathbf{W})^{2} \rightarrow 0$, and $\hat{\tau}^{2} \rightarrow \tau$ a.s.

\section{A.3 Singular vectors of spiked random matrices}

The following is a special case of [BGN12, Theorem 2.9]. An analogue in the spiked covariance model was first established in [Pau07].

Lemma A.3. Under Assumption 5.1, for each $i=1, \ldots, k$ and some choices of signs for $\mathbf{f}_{i}$ and $\mathbf{g}_{i}$, a.s. as $n, d \rightarrow \infty$,

$$
\begin{aligned}
& \sqrt{\gamma} \cdot \lambda_{i} \rightarrow \sqrt{\left(\gamma s_{i}^{2}+1\right)\left(s_{i}^{2}+1\right) / s_{i}^{2}}, \\
& n^{-1} \mathbf{f}_{i}^{\top} \mathbf{u}_{i} \rightarrow \bar{\mu}_{*, i}, \quad d^{-1} \mathbf{g}_{i}^{\top} \mathbf{v}_{i} \rightarrow \mu_{*, i}
\end{aligned}
$$

for the values $\bar{\mu}_{*, i}$ and $\mu_{*, i}$ defined in (2.10). Furthermore, $n^{-1} \mathbf{f}_{i}^{\top} \mathbf{u}_{j} \rightarrow 0$ and $d^{-1} \mathbf{g}_{i}^{\top} \mathbf{v}_{j} \rightarrow 0$ a.s. for all $j \in\{1, \ldots, k\} \backslash\{i\}$.

Proof. Recall

$$
\mathbf{Y}=\frac{1}{n} \mathbf{U} S \mathbf{V}^{\top}+\mathbf{W}
$$

Write $\tilde{\mathbf{U}}=\left(\tilde{\mathbf{u}}_{1}, \ldots, \tilde{\mathbf{u}}_{k}\right) \in \mathbb{R}^{n \times k}$ for the Gram-Schmidt orthogonalization of $\mathbf{U}$, with columns scaled such that $n^{-1}\left\|\tilde{\mathbf{u}}_{i}\right\|^{2}=1$. Assumption 5.1(c) implies that $n^{-1} \mathbf{U}^{\top} \mathbf{U} \rightarrow \operatorname{Id}_{k \times k}$, from which it may be verified that the Gram-Schmidt procedure yields

$$
n^{-1}\|\mathbf{U}-\tilde{\mathbf{U}}\|_{F}^{2} \rightarrow 0
$$

Similarly, letting $\tilde{\mathbf{V}}=\left(\tilde{\mathbf{v}}_{1}, \ldots, \tilde{\mathbf{v}}_{k}\right) \in \mathbb{R}^{d \times k}$ be the Gram-Schmidt orthogonalization of $\mathbf{V}$ scaled such that $d^{-1}\left\|\tilde{\mathbf{v}}_{i}\right\|^{2}=1$, we have

$$
d^{-1}\|\mathbf{V}-\tilde{\mathbf{V}}\|_{F}^{2} \rightarrow 0
$$

Define $\tilde{\mathbf{Y}}=n^{-1} \tilde{\mathbf{U}} S \tilde{\mathbf{V}}^{\top}+\mathbf{W}$, and denote its leading rank- $k$ singular component as

$$
n^{-1} \tilde{\mathbf{F}} \tilde{\Lambda} \tilde{\mathbf{G}}^{\top}=\sum_{i=1}^{n} \frac{\tilde{\lambda}_{i}}{n} \cdot \tilde{\mathbf{f}}_{i} \tilde{\mathbf{g}}_{i}^{\top}
$$

Then (A.3) and (A.4) together imply $\|\tilde{\mathbf{Y}}-\mathbf{Y}\| \rightarrow 0$ in operator norm, so that by the condition of distinct singular values in Assumption 5.1(b) and the Weyl and Davis-Kahan inequalities,

$$
\|S-\tilde{S}\| \rightarrow 0, \quad n^{-1}\|\mathbf{F}-\tilde{\mathbf{F}}\|_{F}^{2} \rightarrow 0, \quad d^{-1}\|\mathbf{G}-\tilde{\mathbf{G}}\|_{F}^{2} \rightarrow 0 .
$$


Combining this with (A.3) and (A.4) and applying Cauchy-Schwarz,

$$
\lim _{n, d \rightarrow \infty} n^{-1} \mathbf{U}^{\top} \mathbf{F}=\lim _{n, d \rightarrow \infty} n^{-1} \tilde{\mathbf{U}}^{\top} \tilde{\mathbf{F}}, \quad \lim _{n, d \rightarrow \infty} d^{-1} \mathbf{V}^{\top} \mathbf{G}=\lim _{n, d \rightarrow \infty} d^{-1} \tilde{\mathbf{V}}^{\top} \tilde{\mathbf{G}} .
$$

[BGN12, Theorem 2.9] applies to describe the almost sure limits of $\tilde{S}, n^{-1} \tilde{\mathbf{U}^{\top}} \tilde{\mathbf{F}}$, and $d^{-1} \tilde{\mathbf{V}}^{\top} \tilde{\mathbf{G}}$. The latter two limits are diagonal with the diagonal entries

$$
n^{-1} \tilde{\mathbf{u}}_{i}^{\top} \tilde{\mathbf{f}}_{i} \rightarrow \sqrt{1-\frac{\left(\gamma+\gamma s_{i}^{2}\right)}{\gamma s_{i}^{2}\left(\gamma s_{i}^{2}+1\right)}}, \quad d^{-1} \tilde{\mathbf{v}}_{i}^{\top} \tilde{\mathbf{g}}_{i} \rightarrow \sqrt{1-\frac{\gamma\left(1+\gamma s_{i}^{2}\right)}{\gamma s_{i}^{2}\left(\gamma s_{i}^{2}+\gamma\right)}} .
$$

These formulas are given by [BGN12, Eqs. 10-11] when $\mathbf{W}$ is Gaussian, with the notational identifications $c \leftrightarrow \gamma, \theta_{i} \leftrightarrow \sqrt{\gamma} \cdot \tilde{s}_{i}$, and a swap of $\mathbf{u}$ with $\mathbf{v}$ because $\mathbf{W}$ in [BGN12] is scaled to have variance $1 / d$ rather than $1 / n$. We have replaced in these limits $\tilde{s}_{i}$ by $s_{i}$, using the above convergence $\|S-\tilde{S}\| \rightarrow 0$. These limits are exactly $\bar{\mu}_{*, i}$ and $\mu_{*, i}$ from (2.10), establishing the lemma.

As the columns of $\mathbf{F}$ and $\mathbf{G}$ are only defined up to an arbitrary sign, we henceforth fix these signs to match those in Lemma A.3. We now prove Proposition 5.2 of the Main Text using the results of [BGN12].

Proof of Proposition 5.2. We show the result for $(\mathbf{V}, \mathbf{G})$; the statement for $(\mathbf{U}, \mathbf{F})$ is analogous. Let $\tilde{\mathbf{V}}=\left(\tilde{\mathbf{v}}_{1}, \ldots, \tilde{\mathbf{v}}_{k}\right) \in \mathbb{R}^{d \times k}$ be the Gram-Schmidt orthogonalization of $\mathbf{V}$, satisfying $d^{-1}\left\|\tilde{\mathbf{v}}_{i}\right\|^{2}=1$ and (A.4). The projections onto and orthogonal to the column span of $\mathbf{V}$ are given by $\mathbf{P}_{\|}=\tilde{\mathbf{V}} \tilde{\mathbf{V}}^{\top} / d$ and $\mathbf{P}_{\perp}=\mathrm{Id}-\tilde{\mathbf{V}} \tilde{\mathbf{V}}^{\top} / d$, respectively. Note that (A.4) and Lemma A.3 imply, almost surely,

$$
\lim _{n, d \rightarrow \infty} d^{-1} \tilde{\mathbf{V}}^{\top} \mathbf{G}=\lim _{n, d \rightarrow \infty} d^{-1} \mathbf{V}^{\top} \mathbf{G}=M_{*}^{\top} .
$$

Then, since $d^{-1} \mathbf{G}^{\top} \mathbf{G}=\mathrm{Id}$, we have

$$
d^{-1}\left(\mathbf{P}_{\perp} \mathbf{G}\right)^{\top}\left(\mathbf{P}_{\perp} \mathbf{G}\right) \rightarrow \mathrm{Id}-M_{*} M_{*}^{\top}=\Sigma_{*} \succ 0 .
$$

This shows that $\mathbf{P}_{\perp} \mathbf{G}$ has full column rank $k$ almost surely for all large $n, d$.

Now let $\tilde{\mathbf{V}}_{\perp}=\left(\tilde{\mathbf{v}}_{1}^{\perp}, \ldots, \tilde{\mathbf{v}}_{k}^{\perp}\right) \in \mathbb{R}^{d \times k}$ be the Gram-Schmidt orthogonalization of $\mathbf{P}_{\perp} \mathbf{G}$, normalized so that $d^{-1}\left\|\tilde{\mathbf{v}}_{i}^{\perp}\right\|^{2}=1$. Then we may represent

$$
\mathbf{G}=\mathbf{P}_{\|} \mathbf{G}+\mathbf{P}_{\perp} \mathbf{G}=\tilde{\mathbf{V}} \Omega+\tilde{\mathbf{V}}_{\perp} \Omega_{\perp}, \quad \Omega=d^{-1} \tilde{\mathbf{V}}^{\top} \mathbf{G}, \quad \Omega_{\perp}=d^{-1} \tilde{\mathbf{V}}_{\perp}^{\top} \mathbf{G} .
$$

Here, almost surely

$$
\Omega \rightarrow M_{*}^{\top}, \quad \Omega_{\perp}^{\top} \Omega_{\perp}=d^{-1}\left(\mathbf{P}_{\perp} \mathbf{G}\right)^{\top}\left(\mathbf{P}_{\perp} \mathbf{G}\right) \rightarrow \Sigma_{*}
$$

so $\Omega_{\perp}$ is invertible for all large $n, d$. Let $\mathbf{Q} \in \mathbb{R}^{d \times d}$ be any orthogonal matrix such that $\mathbf{Q} \tilde{\mathbf{V}}=\tilde{\mathbf{V}}$. Then also $\mathbf{Q V}=\mathbf{V}$, and we have the equality in law

$$
\mathbf{Y} \mathbf{Q}^{\top}=\frac{1}{n} \mathbf{U} S \mathbf{V}^{\top} \mathbf{Q}^{\top}+\mathbf{W} \mathbf{Q}^{\top} \stackrel{L}{=} \frac{1}{n} \mathbf{U} S \mathbf{V}^{\top}+\mathbf{W}=\mathbf{Y}
$$

Since $\mathbf{G}$ contains the leading $k$ right singular vectors of $\mathbf{Y}$, this implies $\mathbf{Q G} \stackrel{L}{=} \mathbf{G}$, so

$$
\tilde{\mathbf{V}} \Omega+\mathbf{Q} \tilde{\mathbf{V}}_{\perp} \Omega_{\perp}=\mathbf{Q G} \stackrel{L}{=} \mathbf{G}=\tilde{\mathbf{V}} \Omega+\tilde{\mathbf{V}}_{\perp} \Omega_{\perp} .
$$


Then $\tilde{\mathbf{V}}_{\perp}$ satisfies the rotational invariance in law $\mathbf{Q} \tilde{\mathbf{V}}_{\perp} \stackrel{L}{=} \tilde{\mathbf{V}}_{\perp}$ for any such $\mathbf{Q}$, so it is Haaruniformly distributed over the Stiefel manifold $\left\{\tilde{\mathbf{V}} \in \mathbb{R}^{d \times k}: \mathbf{V}^{\top} \tilde{\mathbf{V}}_{\perp}=0, d^{-1} \tilde{\mathbf{V}}_{\perp}^{\top} \tilde{\mathbf{V}}_{\perp}=\operatorname{Id}\right\}$. Letting $\mathbf{Z} \in \mathbb{R}^{d \times k}$ be a random matrix with i.i.d. entries $Z_{i j} \sim \mathcal{N}(0,1)$, this means that we can construct $\tilde{\mathbf{V}}_{\perp}=\mathbf{P}_{\perp} \mathbf{Z} \Sigma^{-1 / 2}$, where $\Sigma=\mathbf{Z}^{\top} \mathbf{P}_{\perp} \mathbf{Z} / d$. Then

$$
\mathbf{G}=\tilde{\mathbf{V}} \Omega+\mathbf{P}_{\perp} \mathbf{Z} \Sigma^{-1 / 2} \Omega_{\perp} .
$$

By the law of large numbers, $\Sigma \rightarrow \operatorname{Id}_{k \times k}$ and $d^{-1}\left\|\mathbf{P}_{\perp} \mathbf{Z}-\mathbf{Z}\right\|_{F}^{2}=d^{-1}\left\|\tilde{\mathbf{V}}\left(\tilde{\mathbf{V}}^{\top} \mathbf{Z}\right) / d\right\|_{F}^{2} \rightarrow 0$ almost surely. Thus

$$
d^{-1}\left\|\mathbf{G}-\tilde{\mathbf{V}} \Omega-\mathbf{Z} \Omega_{\perp}\right\|_{F}^{2} \rightarrow 0 .
$$

Applying (A.4) and (A.6), we then have $(\mathbf{V}, \mathbf{G}) \stackrel{W_{2}}{\rightarrow}(V, G)$ where $G=M_{*} V+\Sigma_{*}^{1 / 2} Z$ and $Z \sim$ $\mathcal{N}(0$, Id $)$, as desired.

\section{B Analysis of the NPMLE}

For $M \in \mathbb{R}^{k \times k}$ invertible and $\Sigma \in \mathbb{R}^{k \times k}$ symmetric positive-definite, consider the compound decision model $\Theta \sim \pi$ and $X \mid \Theta \sim \mathcal{N}(M \cdot \Theta, \Sigma)$. Denote the Lebesgue density function of $\mathcal{N}(0, \Sigma)$ by

$$
\phi_{\Sigma}(x)=\frac{1}{(2 \pi)^{k / 2}|\Sigma|^{1 / 2}} \exp \left(-\frac{1}{2} \cdot x^{\top} \Sigma^{-1} x\right) .
$$

Then the marginal density of $X$ is

$$
f_{(M \cdot \pi) * \mathcal{N}(0, \Sigma)}(x)=\mathbb{E}\left[\phi_{\Sigma}(x-M \Theta)\right]=\int \phi_{\Sigma}(x-M \theta) \mathrm{d} \pi(\theta) .
$$

Noting that $\mathrm{d} \phi_{\Sigma}(x)=-x^{\top} \Sigma^{-1} \cdot \phi_{\Sigma}(x)$, we have

$$
\begin{aligned}
\frac{\mathrm{d} f_{(M \cdot \pi) * \mathcal{N}(0, \Sigma)}^{\top}}{f_{(M \cdot \pi) * \mathcal{N}(0, \Sigma)}}(x) & =\frac{\mathbb{E}\left[-\Sigma^{-1}(x-M \Theta) \cdot \phi_{\Sigma}(x-M \Theta)\right]}{\mathbb{E}\left[\phi_{\Sigma}(x-M \Theta)\right]} \\
& =-\Sigma^{-1} x+\Sigma^{-1} M \cdot \frac{\mathbb{E}\left[\Theta \cdot \phi_{\Sigma}(x-M \Theta)\right]}{\mathbb{E}\left[\phi_{\Sigma}(x-M \Theta)\right]}
\end{aligned}
$$

Thus we obtain Tweedie's form of the posterior mean denoiser,

$$
\theta(x \mid M, \Sigma, \pi)=\frac{\mathbb{E}\left[\Theta \cdot \phi_{\Sigma}(x-M \Theta)\right]}{\mathbb{E}\left[\phi_{\Sigma}(x-M \Theta)\right]}=M^{-1}\left(x+\Sigma \cdot \frac{\mathrm{d} f_{(M \cdot \pi) * \mathcal{N}(0, \Sigma)}^{\top}}{f_{(M \cdot \pi) * \mathcal{N}(0, \Sigma)}}(x)\right) .
$$

Assumption 5.1(d) guarantees a uniform Lipschitz property of $\theta(\cdot)$ in a neighborhood of $\pi_{*}$. This in fact implies a uniform Lipschitz property in a neighborhood of $\left(M_{*}, \Sigma_{*}, \pi_{*}\right)$-we record this fact in the following proposition.

Proposition B.1. Under Assumption 5.1(d), there is an open neighborhood $O$ of $\left(M_{*}, \Sigma_{*}, \pi_{*}\right)$ (weakly with respect to $\pi_{*}$ ) such that $\theta(x \mid M, \Sigma, \pi)$ is Lipschitz in $x$ uniformly over $(M, \Sigma, \pi) \in O$.

Proof. For any $(M, \Sigma, \pi) \in O$, define $\tilde{\pi}=M_{*}^{-1} \Sigma_{*}^{1 / 2} \Sigma^{-1 / 2} M \cdot \pi$. Then

$$
\begin{aligned}
\theta(x \mid M, \Sigma, \pi) & =\mathbb{E}_{\Theta \sim \pi}\left[\Theta \mid M \Theta+\Sigma^{1 / 2} Z=x\right] \\
& =\mathbb{E}_{\Theta \sim \pi}\left[\Theta \mid \Sigma_{*}^{1 / 2} \Sigma^{-1 / 2} M \Theta+\Sigma_{*}^{1 / 2} Z=\Sigma_{*}^{1 / 2} \Sigma^{-1 / 2} x\right] \\
& =\underbrace{\left(M_{*}^{-1} \Sigma_{*}^{1 / 2} \Sigma^{-1 / 2} M\right)^{-1}}_{K_{1}} \mathbb{E}_{\Theta \sim \tilde{\pi}}[\Theta \mid M_{*} \Theta+\Sigma_{*}^{1 / 2} Z=\underbrace{\Sigma_{*}^{1 / 2} \Sigma^{-1 / 2}}_{K_{2}} x] \\
& =K_{1} \theta\left(K_{2} x \mid M_{*}, \Sigma_{*}, \tilde{\pi}\right) .
\end{aligned}
$$


Since $\theta\left(x \mid M_{*}, \Sigma_{*}, \tilde{\pi}\right)$ is Lipschitz in $x$ uniformly over all $\tilde{\pi}$ in an open neighborhood of $\pi_{*}$ by Assumption 5.1(d), the result follows for a sufficiently small neighborhood $O$ of $\left(M_{*}, \Sigma_{*}, \pi_{*}\right)$.

\section{B.1 Consistency of the NPMLE}

The entrywise Gaussian approximations of (2.13) and (2.18) for the sample PCs and AMP iterates will hold in the sense of empirical Wasserstein convergence as $n, d \rightarrow \infty$. Thus we first show a consistency result for the NPMLE in this setting, where $\mathbf{X} \mid \boldsymbol{\Theta}$ only approximately follows this Gaussian model.

Lemma B.2. Fix $k \geq 1, M_{*} \in \mathbb{R}^{k \times k}$ non-singular, and $\Sigma_{*} \in \mathbb{R}^{k \times k}$ symmetric positive-definite. Let $\mathcal{P}$ be a class of distributions on $\mathbb{R}^{k}$ satisfying Assumption 5.1, and fix $\pi_{*} \in \mathcal{P}$. As $n \rightarrow \infty$, let $\boldsymbol{\Theta}, \mathbf{X} \in \mathbb{R}^{n \times k}$ satisfy

$$
(\Theta, \mathbf{X}) \stackrel{W_{2}}{\rightarrow}(\Theta, X)
$$

where $\Theta \sim \pi_{*}$ and $X \mid \Theta \sim \mathcal{N}\left(M_{*} \cdot \Theta, \Sigma_{*}\right)$, and let $M_{n}, \Sigma_{n} \in \mathbb{R}^{k \times k}$ satisfy $M_{n} \rightarrow M_{*}$ and $\Sigma_{n} \rightarrow \Sigma_{*}$.

Let $x_{1}, \ldots, x_{n}$ be the rows of $\mathbf{X}$. Suppose $\pi_{n} \in \mathcal{P}$ is any approximate NPMLE in the sense

$$
\liminf _{n \rightarrow \infty} \frac{1}{n} \sum_{i=1}^{n} \log \frac{f_{\left(M_{n} \cdot \pi_{n}\right) * \mathcal{N}\left(0, \Sigma_{n}\right)}\left(x_{i}\right)}{f_{\left(M_{*} \cdot \pi_{*}\right) * \mathcal{N}\left(0, \Sigma_{*}\right)}\left(x_{i}\right)} \geq 0 .
$$

Then $\pi_{n}$ converges weakly to $\pi_{*}$ as $n \rightarrow \infty$.

This has the following oracle implication for the corresponding empirical Bayes estimator of $\Theta$ :

Corollary B.3. In the setting of Lemma B.2, let $\theta\left(\mathbf{X} \mid M_{*}, \Sigma_{*}, \pi_{*}\right)$ be the Bayes posterior mean function as defined in (2.7). Then

$$
\frac{1}{n}\left\|\theta\left(\mathbf{X} \mid M_{*}, \Sigma_{*}, \pi_{*}\right)-\theta\left(\mathbf{X} \mid M_{n}, \Sigma_{n}, \pi_{n}\right)\right\|_{F}^{2} \rightarrow 0
$$

Consequently

$$
\frac{1}{n}\left\|\theta\left(\mathbf{X} \mid M_{n}, \Sigma_{n}, \pi_{n}\right)-\Theta\right\|_{F}^{2} \rightarrow \operatorname{mmse}\left(\pi_{*} \mid M_{*}, \Sigma_{*}\right) .
$$

Thus the empirical Bayes estimate of $\Theta$ achieves the oracle asymptotic Bayes risk of the true prior $\pi_{*}$, as long as the empirical convergence $(\Theta, \mathbf{X}) \stackrel{W_{2}}{\rightarrow}(\Theta, X)$ holds, and $M_{n}, \Sigma_{n}$ are consistent estimates of the parameters $M_{*}, \Sigma_{*}$ describing the conditional Gaussian law of $X \mid \Theta$.

The proofs of these results follow the ideas of [Zha09, JZ09, SG20]. Our analyses are simpler, as we do not assume a particular rate of convergence in (B.3), and we correspondingly do not study the convergence rate of $\pi_{n}$ to $\pi_{*}$.

Proof of Lemma B.2. Write as shorthand

$$
f_{*}(x)=f_{\left(M_{*} \cdot \pi_{*}\right) * \mathcal{N}\left(0, \Sigma_{*}\right)}(x), \quad f_{n}(x)=f_{\left(M_{n} \cdot \pi_{n}\right) * \mathcal{N}\left(0, \Sigma_{n}\right)}(x),
$$

and denote their squared Hellinger distance by $d_{H}\left(f_{n}, f_{*}\right)^{2}=1-\int \sqrt{f_{n}(x) f_{*}(x)} \mathrm{d} x$. We show that

$$
d_{H}\left(f_{n}, f_{*}\right)^{2} \rightarrow 0
$$

as $n \rightarrow \infty$. Fix any $\varepsilon>0$, and suppose by contradiction that $\lim _{\sup _{n \rightarrow \infty}} d_{H}\left(f_{n}, f_{*}\right)^{2}>\varepsilon$. This implies that along some subsequence $\left\{n_{m}\right\}_{m=1}^{\infty}$, the function $f_{n_{m}}$ belongs to the function class

$$
\mathcal{F}=\left\{f_{(M \cdot \pi) * \mathcal{N}(0, \Sigma)}: d_{H}\left(f_{(M \cdot \pi) * \mathcal{N}(0, \Sigma)}, f_{*}\right)^{2}>\varepsilon, \pi \in \mathcal{P},\left\|M-M_{*}\right\|<\left\|M_{*}\right\| / 2,\left\|\Sigma-\Sigma_{*}\right\|<\left\|\Sigma_{*}\right\| / 2\right\} .
$$


We denote by $\underline{\lim } a_{n} \equiv \liminf _{m \rightarrow \infty} a_{n_{m}}$ and $\varlimsup a_{n} \equiv \limsup _{m \rightarrow \infty} a_{n_{m}}$ the limits along this subsequence.

Observe that from the form of (B.1), the functions $f \in \mathcal{F}$ are uniformly bounded by a constant $C_{0}>0$ and are equicontinuous. Consider two constants $B, \eta>0$. Let $\mathbb{B}^{k}(B)=\left\{x \in \mathbb{R}^{k}:\|x\| \leq B\right\}$, and define the semi-norm

$$
\|f\|_{\infty, B}=\sup _{x \in \mathbb{B}^{k}(B)}|f(x)| .
$$

Then by Arzelà-Ascoli, there exists a finite ( $n$-independent) $\eta$-cover $\mathcal{C} \subset \mathcal{F}$ in this semi-norm: for any $f \in \mathcal{F}$, there is some $g \in \mathcal{C}$ where $\|f-g\|_{\infty, B}<\eta$. We define the smoothed indicator function

$$
\mathfrak{f}(x)= \begin{cases}\eta & \text { if } x \in \mathbb{B}^{k}(B) \\ \eta \cdot(B /\|x\|)^{k+1} & \text { if } x \notin \mathbb{B}^{k}(B) .\end{cases}
$$

For all large $n_{m}$, letting $g_{n_{m}} \in \mathcal{C}$ be such that $\left\|f_{n_{m}}-g_{n_{m}}\right\|_{\infty, B}<\eta$, we then have the pointwise bounds

$$
f_{n_{m}}(x) \leq \begin{cases}g_{n_{m}}(x)+\mathfrak{f}(x) & \text { if } x \in \mathbb{B}^{k}(B) \\ C_{0} & \text { if } x \notin \mathbb{B}^{k}(B) .\end{cases}
$$

The given condition that $\pi_{n}$ is an approximate NPMLE implies that

$$
\begin{aligned}
0 \leq \underline{\lim } \frac{1}{n} \sum_{i=1}^{n} \log \frac{f_{n}\left(x_{i}\right)}{f_{*}\left(x_{i}\right)} & \leq \varlimsup \\
& =(\mathrm{I})+(\mathrm{II}) .
\end{aligned}
$$

For $(\mathrm{I})$, observe that since $\mathcal{C}$ is a finite $n$-independent cover, we have

$$
(\mathrm{I}) \leq \max _{g \in \mathcal{C}} \varlimsup \frac{1}{n} \sum_{i=1}^{n} \log \frac{g\left(x_{i}\right)+\mathfrak{f}\left(x_{i}\right)}{f_{*}\left(x_{i}\right)} .
$$

For any $f=f_{(M \cdot \pi) * \mathcal{N}(0, \Sigma)} \in \mathcal{F}$, its posterior mean denoising function $\theta(x \mid M, \Sigma, \pi)$ is given by (B.2). Then applying Assumption 5.1(d), for each fixed $f \in \mathcal{F}$ and a constant $C_{f}>0$, we have

$$
\left\|\mathrm{d}^{2} \log f(x)\right\|=\left\|\Sigma^{-1} \cdot(\operatorname{Id}+M \cdot \mathrm{d} \theta(x \mid M, \Sigma, \pi))\right\|<C_{f} .
$$

Thus $\log f_{*} \in \operatorname{PL}(2)$ and $\log g \in \operatorname{PL}(2)$ for all $g \in \mathcal{C}$. It may be checked that $\log \mathfrak{f} \in \operatorname{PL}(2)$, and since $(a, b) \mapsto \log \left(e^{a}+e^{b}\right)$ is Lipschitz, this implies that $x \mapsto \log (g(x)+\mathfrak{f}(x))$ also belongs to $\operatorname{PL}(2)$. Then by the convergence $\mathbf{X} \stackrel{W_{2}}{\rightarrow} X \sim f_{*}$, we obtain

$$
\text { (I) } \leq \max _{g \in \mathcal{C}} \mathbb{E}_{X \sim f_{*}}\left[\log \frac{g(X)+\mathfrak{f}(X)}{f_{*}(X)}\right] .
$$

For each $g \in \mathcal{C}$, applying $(\log x) / 2=\log \sqrt{x} \leq \sqrt{x}-1, \sqrt{x+y} \leq \sqrt{x}+\sqrt{y}$, and Cauchy-Schwarz,

$$
\begin{aligned}
\frac{1}{2} \mathbb{E}_{X \sim f_{*}}\left[\log \frac{g(X)+\mathfrak{f}(X)}{f_{*}(X)}\right] & \leq \mathbb{E}_{X \sim f_{*}}\left[\sqrt{\left.\frac{g(X)+\mathfrak{f}(X)}{f_{*}(X)}\right]}-1\right. \\
& =\int \sqrt{(g(x)+\mathfrak{f}(x)) f_{*}(x)} d x-1 \\
& \leq \int \sqrt{g(x) f_{*}(x)} d x-1+\int \sqrt{\mathfrak{f}(x) f_{*}(x)} d x \\
& \leq-d_{H}\left(g, f_{*}\right)^{2}+\sqrt{\int \mathfrak{f}(x) d x .}
\end{aligned}
$$


The condition $g \in \mathcal{C} \subset \mathcal{F}$ implies $d_{H}\left(g, f_{*}\right)^{2}>\varepsilon$. Furthermore, $\int \mathfrak{f}(x) d x=\int \mathfrak{f}(B y) B^{k} d y=C_{k} \eta \cdot B^{k}$ for a constant $C_{k}>0$. Thus $(\mathrm{I}) \leq-2 \varepsilon+2 \sqrt{C_{k} \eta \cdot B^{k}}$.

For (II), let us set

$$
\psi_{\eta, B}(x)=\log \max \left(\frac{C_{0}\|x\|^{k+1}}{\eta B^{k+1}}, 1\right) .
$$

Then clearly $\psi_{\eta, B} \in \mathrm{PL}(2)$, so

$$
(\mathrm{II}) \leq \varlimsup \frac{1}{n} \sum_{i: x_{i} \notin \mathbb{B}^{k}(B)} \log \frac{C_{0}}{\mathfrak{f}\left(x_{i}\right)} \leq \varlimsup \frac{1}{n} \sum_{i=1}^{n} \psi_{\eta, B}\left(x_{i}\right)=\mathbb{E}_{X \sim f_{*}}\left[\psi_{\eta, B}(X)\right] .
$$

Combining with the above bound for (I), we obtain

$$
0 \leq-2 \varepsilon+2 \sqrt{C_{k} \eta \cdot B^{k}}+\mathbb{E}_{X \sim f_{*}}\left[\psi_{\eta, B}(X)\right] .
$$

This must hold for all $B, \eta>0$. However, taking $B \rightarrow \infty$ and $\eta \rightarrow 0$ such that $\eta B^{k+1} \rightarrow \infty$ but $\eta B^{k} \rightarrow 0$, we have $2 \sqrt{C_{k} \eta \cdot B^{k}} \rightarrow 0$ and $\mathbb{E}_{X \sim f_{*}}\left[\psi_{\eta, B}(X)\right] \rightarrow 0$ by the dominated convergence theorem. This yields a contradiction for sufficiently large $B$ and small $\eta$. Then we must have $\lim \sup _{n \rightarrow \infty} d_{H}\left(f_{n}, f_{*}\right)^{2} \leq \varepsilon$. Here $\varepsilon>0$ is arbitrary, so we have shown (B.6).

This implies the weak convergence of $\left(M_{n} \cdot \pi_{n}\right) * \mathcal{N}\left(0, \Sigma_{n}\right)$ to $\left(M_{*} \cdot \pi_{*}\right) * \mathcal{N}\left(0, \Sigma_{*}\right)$. If $\psi_{n}$ and $\psi_{*}$ are the characteristic functions of $\pi_{n}$ and $\pi_{*}$, then for all $t \in \mathbb{R}^{k}$,

$$
\psi_{n}\left(M_{n}^{\top} t\right) \cdot e^{-\frac{t^{\top} \Sigma_{n} t}{2}} \rightarrow \psi_{*}\left(M_{*}^{\top} t\right) \cdot e^{-\frac{t^{\top} \Sigma_{*} t}{2}}
$$

as the left side is the characteristic function of $\left(M_{n} \cdot \pi_{n}\right) * \mathcal{N}\left(0, \Sigma_{n}\right)$ while the right side is that of $\left(M_{*} \cdot \pi_{*}\right) * \mathcal{N}\left(0, \Sigma_{*}\right)$. Since $\Sigma_{n} \rightarrow \Sigma_{*}$ and $M_{n} \rightarrow M_{*}$, this implies $\psi_{n}\left(M_{*}^{\top} t\right) \rightarrow \psi_{*}\left(M_{*}^{\top} t\right)$. Then $\psi_{n}(t) \rightarrow \psi_{*}(t)$ for all $t \in \mathbb{R}^{k}$, because $M_{*}$ is invertible. So $\pi_{n}$ converges weakly to $\pi_{*}$.

\section{B.2 Bayes posterior mean and its derivatives}

We now show Corollary B.3 and an analogous statement for the derivative of $\theta(\cdot)$.

Lemma B.4. Let $\pi_{n}, \pi_{*}$ be probability distributions on $\mathbb{R}^{k}$ such that $\pi_{n}$ converges weakly to $\pi_{*}$. Let $g_{n}, g_{*}: \mathbb{R}^{k} \times \mathbb{R}^{k} \rightarrow \mathbb{R}^{m}$ be such that

(a) For any $B>0, g_{n}(x, \theta) \rightarrow g_{*}(x, \theta)$ uniformly over $(x, \theta) \in \mathbb{R}^{k} \times \mathbb{B}^{k}(B)$.

(b) For some constant $C_{0}>0$ and all $(x, \theta) \in \mathbb{R}^{k} \times \mathbb{R}^{k},\left\|g_{*}(x, \theta)\right\|<C_{0}$ and $\left\|g_{n}(x, \theta)\right\|<C_{0}$.

(c) For any $B>0$, some constant $L_{B}>0$, and all $(x, \theta) \in \mathbb{R}^{k} \times \mathbb{B}^{k}(B),\left\|\mathrm{d}_{\theta} g_{*}(x, \theta)\right\|<L_{B}$.

Then as $n \rightarrow \infty$,

$$
\sup _{x \in \mathbb{R}^{k}}\left\|\int g_{n}(x, \theta) \mathrm{d} \pi_{n}(\theta)-\int g_{*}(x, \theta) \mathrm{d} \pi_{*}(\theta)\right\| \rightarrow 0
$$

Proof. Fix $\varepsilon>0$. Then there is some $B>0$ for which $\pi_{*}\left(\mathbb{R}^{k} \backslash \mathbb{B}^{k}(B)\right) \leq \varepsilon$ and $\pi_{n}\left(\mathbb{R}^{k} \backslash \mathbb{B}^{k}(B)\right) \leq \varepsilon$ for all $n$. Conditions (b) and (c) imply that the functions $\theta \mapsto g_{*}(x, \theta)$ are uniformly bounded and equicontinuous over $\theta \in \mathbb{B}^{k}(B)$, for all $x \in \mathbb{R}^{k}$. Then by Arzelà-Ascoli, there is a finite set $\mathcal{C}$ of continuous bounded functions on $\mathbb{B}^{k}(B)$ such that for each $x \in \mathbb{R}^{k}$, there exists $h_{x} \in \mathcal{C}$ for which

$$
\sup _{\theta \in \mathbb{B}^{k}(B)}\left\|g_{*}(x, \theta)-h_{x}(\theta)\right\| \leq \varepsilon
$$


Let us write

$$
\sup _{x \in \mathbb{R}^{k}}\left\|\int g_{n}(x, \theta) \mathrm{d} \pi_{n}(\theta)-\int g_{*}(x, \theta) \mathrm{d} \pi_{*}(\theta)\right\| \leq R_{1}+R_{2}+R_{3}+R_{4}
$$

where

$$
\begin{aligned}
& R_{1}=\sup _{x \in \mathbb{R}^{k}} \int_{\mathbb{R}^{k} \backslash \mathbb{B}^{k}(B)}\left\|g_{n}(x, \theta)\right\| \mathrm{d} \pi_{n}(\theta)+\int_{\mathbb{R}^{k} \backslash \mathbb{B}^{k}(B)}\left\|g_{*}(x, \theta)\right\| \mathrm{d} \pi_{*}(\theta) \\
& R_{2}=\sup _{x \in \mathbb{R}^{k}} \int_{\mathbb{B}^{k}(B)}\left\|g_{n}(x, \theta)-g_{*}(x, \theta)\right\| \mathrm{d} \pi_{n}(\theta)+\int_{\mathbb{B}^{k}(B)}\left\|g_{n}(x, \theta)-g_{*}(x, \theta)\right\| \mathrm{d} \pi_{*}(\theta) \\
& R_{3}=\sup _{x \in \mathbb{R}^{k}} \int_{\mathbb{B}^{k}(B)}\left\|g_{*}(x, \theta)-h_{x}(\theta)\right\| \mathrm{d} \pi_{n}(\theta)+\int_{\mathbb{B}^{k}(B)}\left\|g_{*}(x, \theta)-h_{x}(\theta)\right\| \mathrm{d} \pi_{*}(\theta) \\
& R_{4}=\sup _{h \in \mathcal{C}}\left|\int_{\mathbb{B}^{k}(B)} h(\theta) \mathrm{d} \pi_{n}(\theta)-\int_{\mathbb{B}^{k}(B)} h(\theta) \mathrm{d} \pi_{*}(\theta)\right| .
\end{aligned}
$$

We have $R_{1} \leq 2 C_{0} \varepsilon$ by condition (b), and $R_{3} \leq 2 \varepsilon$. The uniform convergence of condition (a) implies $R_{2} \rightarrow 0$. Since $\mathcal{C}$ is a finite set and each $h \in \mathcal{C}$ is continuous and bounded, $R_{4} \rightarrow 0$. As $\varepsilon>0$ is arbitrary, this shows the lemma.

Corollary B.5. In the setting of Lemma B.2, denote

$$
f_{n}(x)=f_{\left(M_{n} \cdot \pi_{n}\right) * \mathcal{N}\left(0, \Sigma_{n}\right)}(x), \quad f_{*}(x)=f_{\left(M_{*} \cdot \pi_{*}\right) * \mathcal{N}\left(0, \Sigma_{*}\right)}(x) .
$$

Then as $n \rightarrow \infty$, for each fixed $j \geq 1$

$$
\sup _{x \in \mathbb{R}^{k}}\left|f_{n}(x)-f_{*}(x)\right| \rightarrow 0, \quad \sup _{x \in \mathbb{R}^{k}}\left\|\mathrm{~d}^{j} f_{n}(x)-\mathrm{d}^{j} f_{*}(x)\right\| \rightarrow 0 .
$$

Proof. Lemma B.2 shows that $\pi_{n} \rightarrow \pi_{*}$ weakly. Consider the functions $g_{n}(x, \theta)=\phi_{\Sigma_{n}}\left(x-M_{n} \theta\right)$ and $g_{*}(x, \theta)=\phi_{\Sigma_{*}}\left(x-M_{*} \theta\right)$. From the form (B.1), it suffices to show that the conditions of Lemma B.4 hold for $g_{n} \rightarrow g_{*}$ and also for $\mathrm{d}_{x}^{j} g_{n} \rightarrow \mathrm{d}_{x}^{j} g_{*}$. Observe that for any fixed integers $a, b, c \geq 0$,

$$
\|x-M \theta\|^{a}\|M\|^{b}\left\|\Sigma^{-1}\right\|^{c} \cdot \phi_{\Sigma}(x-M \theta)
$$

is uniformly bounded over all $(x, \theta) \in \mathbb{R}^{k} \times \mathbb{R}^{k}$ and all $M, \Sigma$ in a sufficiently small neighborhood of $M_{*}, \Sigma_{*}$. Then differentiating $g(x, \theta)=\phi_{\Sigma}(x-M \theta)$ by the chain rule, the derivative of any fixed orders in $(x, \theta)$ is uniformly bounded, checking conditions (b) and (c) of Lemma B.4. Furthermore, this shows that the derivative of $g(x, \theta)$ of any fixed orders in $(x, \theta, M, \Sigma)$ is uniformly bounded over all $x \in \mathbb{R}^{k}$, all $(M, \Sigma)$ in a neighborhood of $\left(M_{*}, \Sigma_{*}\right)$, and all $\theta$ in any compact ball $\mathbb{B}^{k}(B)$. This implies $g_{n} \rightarrow g_{*}$ and $\mathrm{d}_{x}^{j} g_{n} \rightarrow \mathrm{d}_{x}^{j} g_{*}$ uniformly over $(x, \theta) \in \mathbb{R}^{k} \times \mathbb{B}^{k}(B)$, checking condition (a).

Proof of Corollary B.3. Let $f_{*}=f_{\left(M_{*} \cdot \pi_{*}\right) * \mathcal{N}\left(0, \Sigma_{*}\right)}$ and $f_{n}=f_{\left(M_{n} \cdot \pi_{n}\right) * \mathcal{N}\left(0, \Sigma_{n}\right)}$. From Tweedie's formula ((B.2)),

$$
\theta\left(x \mid M_{*}, \Sigma_{*}, \pi_{*}\right)=M_{*}^{-1}\left(\Sigma_{*} \frac{\mathrm{d} f_{*}^{\top}}{f_{*}}(x)+x\right), \quad \theta\left(x \mid M_{n}, \Sigma_{n}, \pi_{n}\right)=M_{n}^{-1}\left(\Sigma_{n} \frac{\mathrm{d} f_{n}^{\top}}{f_{n}}(x)+x\right) .
$$


Let us write $g_{*}=\mathrm{d} f_{*} / f_{*}$ and $g_{n}=\mathrm{d} f_{n} / f_{n}$. Then

$$
\begin{aligned}
& \frac{1}{n}\left\|\theta\left(\mathbf{X} \mid M_{*}, \Sigma_{*}, \pi_{*}\right)-\theta\left(\mathbf{X} \mid M_{n}, \Sigma_{n}, \pi_{n}\right)\right\|_{F}^{2} \\
& \leq \frac{3}{n} \sum_{i=1}^{n}\left\|M_{*}^{-1} \Sigma_{*}\right\|^{2} \cdot\left\|g_{*}\left(x_{i}\right)-g_{n}\left(x_{i}\right)\right\|^{2} \\
& \quad+\frac{3}{n} \sum_{i=1}^{n}\left\|M_{n}^{-1} \Sigma_{n}-M_{*}^{-1} \Sigma_{*}\right\|^{2} \cdot\left\|g_{n}\left(x_{i}\right)\right\|^{2}+\frac{3}{n} \sum_{i=1}^{n}\left\|M_{n}^{-1}-M_{*}^{-1}\right\|^{2} \cdot\left\|x_{i}\right\|^{2} \\
& =R_{1}+R_{2}+R_{3} .
\end{aligned}
$$

Note that $M_{n} \rightarrow M_{*}$ and $n^{-1} \sum_{i}\left\|x_{i}\right\|^{2}<C$ for a constant $C>0$ and all large $n$, by the assumption $\mathbf{X} \stackrel{W_{2}}{\rightarrow} X$. Thus $R_{3} \rightarrow 0$. By Proposition B.1, $\theta\left(x \mid M_{n}, \Sigma_{n}, \pi_{n}\right)$ and thus $g_{n}(x)$ are both Lipschitz in $x$, uniformly for all large $n$. So also $n^{-1} \sum_{i}\left\|g_{n}\left(x_{i}\right)\right\|^{2}<C$ for a constant $C>0$ and all large $n$. Then since $\Sigma_{n} \rightarrow \Sigma_{*}$, we have $R_{2} \rightarrow 0$.

To show $R_{1} \rightarrow 0$, fix any $B>0$ and apply $g_{*}-g_{n}=\left(f_{n}-f_{*}\right) \mathrm{d} f_{*} /\left(f_{*} f_{n}\right)+\left(\mathrm{d} f_{*}-\mathrm{d} f_{n}\right) / f_{n}$ to write

$$
\begin{aligned}
R_{1} \leq \frac{C}{n} \sum_{i=1}^{n}\left\|\frac{\left(f_{n}-f_{*}\right) \mathrm{d} f_{*}}{f_{*} f_{n}}\left(x_{i}\right)+\frac{\mathrm{d} f_{*}-\mathrm{d} f_{n}}{f_{n}}\left(x_{i}\right)\right\|^{2} \mathbb{1}\left\{x_{i} \in \mathbb{B}^{k}(B)\right\} \\
\quad+\frac{C}{n} \sum_{i=1}^{n}\left(\left\|g_{*}\left(x_{i}\right)\right\|^{2}+\left\|g_{n}\left(x_{i}\right)\right\|^{2}\right) \mathbb{1}\left\{x_{i} \notin \mathbb{B}^{k}(B)\right\} \equiv(\mathrm{I})+(\mathrm{II}) .
\end{aligned}
$$

We have the bounds $f_{*}(x)>c_{B}$ and $\left\|\mathrm{d} f_{*}(x)\right\|<C_{B}$ for some constants $C_{B}, c_{B}>0$ and all $x \in \mathbb{B}^{k}(B)$. Combining with the uniform convergence in Corollary B.5, this yields

$$
(\mathrm{I}) \leq C_{B}^{\prime} \cdot \sup _{x \in \mathbb{B}^{k}(B)}\left|f_{n}(x)-f_{*}(x)\right|+\left\|\mathrm{d} f_{n}(x)-\mathrm{d} f_{*}(x)\right\| \rightarrow 0
$$

as $n \rightarrow \infty$. For (II), applying again that $g_{*}$ and $g_{n}$ are uniformly Lipschitz in $x$ for large $n$, for some constant $C>0$ (independent of $B$ ) we have

$$
\left\|g_{*}\left(x_{i}\right)\right\| \leq\left\|g_{*}(0)\right\|+C\left\|x_{i}\right\|, \quad\left\|g_{n}\left(x_{i}\right)\right\| \leq\left\|g_{n}(0)\right\|+C\left\|x_{i}\right\| .
$$

Then applying $g_{n}(0) \rightarrow g_{*}(0)$ as $n \rightarrow \infty$, this yields for some constants $C, C^{\prime}>0$ that

$$
(\mathrm{II}) \leq \frac{C}{n} \sum_{i=1}^{n}\left(C^{\prime}+\left\|x_{i}\right\|^{2}\right) \mathbb{1}\left\{x_{i} \notin \mathbb{B}^{k}(B)\right\} .
$$

Define

$$
h(x)= \begin{cases}C^{\prime}+\|x\|^{2} & \text { if } x \notin \mathbb{B}^{k}(B) \\ B^{2}\left(1-\frac{B-1}{\|x\|^{2}}\right)\left(C^{\prime}+\|x\|^{2}\right) & \text { if } x \in \mathbb{B}^{k}(B) \backslash \mathbb{B}^{k}(B-1) \\ 0 & \text { if } x \in \mathbb{B}^{k}(B-1) .\end{cases}
$$

Then $h \in \mathrm{PL}(2)$, and (II) $\leq n^{-1} \sum_{i} h\left(x_{i}\right) \rightarrow \mathbb{E}_{X \sim f_{*}}[h(X)]$. Combining with the bound for (I), $\lim \sup _{n \rightarrow \infty} R_{1} \leq \mathbb{E}_{X \sim f_{*}}[h(X)]$. Taking $B \rightarrow \infty$ and applying the dominated convergence theorem, we obtain $R_{1} \rightarrow 0$, and this completes the proof of (B.5).

As $\theta\left(x \mid M_{*}, \Sigma_{*}, \pi_{*}\right)$ is Lipschitz in $x$ by Assumption $5.1(\mathrm{~d})$ and $(\Theta, \mathbf{X}) \stackrel{W_{2}}{\rightarrow}(\Theta, X)$,

$$
\frac{1}{n}\left\|\theta\left(\mathbf{X} \mid M_{*}, \Sigma_{*}, \pi_{*}\right)-\Theta\right\|_{F}^{2} \rightarrow \mathbb{E}\left[\|\Theta-\mathbb{E}[\Theta \mid X]\|^{2}\right]=\operatorname{mmse}\left(\pi \mid M_{*}, \Sigma_{*}\right),
$$

which immediately implies the second part of the corollary. 
Proposition B.6. In the setting of Lemma B.2, as $n \rightarrow \infty$,

$$
\frac{1}{n} \sum_{i=1}^{n}\left\|\mathrm{~d} \theta\left(x_{i} \mid M_{*}, \Sigma_{*}, \pi_{*}\right)-\mathrm{d} \theta\left(x_{i} \mid M_{n}, \Sigma_{n}, \pi_{n}\right)\right\| \rightarrow 0 .
$$

Proof. The proof is similar to that of Corollary B.3 above. Differentiating (B.7) again in $x$,

$$
\mathrm{d} \theta\left(x \mid M_{*}, \Sigma_{*}, \pi_{*}\right)=M_{*}^{-1}\left(\Sigma_{*}\left(\frac{\mathrm{d}^{2} f_{*}}{f_{*}}(x)-\frac{\mathrm{d} f_{*} \otimes \mathrm{d} f_{*}}{f_{*}^{2}}(x)\right)+\mathrm{Id}\right),
$$

and similarly for $\mathrm{d} \theta\left(x \mid M_{n}, \Sigma_{n}, \pi_{n}\right)$. Let us set $g_{*}=\mathrm{d}^{2} f_{*} / f_{*}-\mathrm{d} f_{*} \otimes \mathrm{d} f_{*} / f_{*}^{2}$ and $g_{n}=\mathrm{d}^{2} f_{n} / f_{n}-$ $\mathrm{d} f_{n} \otimes \mathrm{d} f_{n} / f_{n}^{2}$. Then

$$
\begin{aligned}
& \frac{1}{n} \sum_{i=1}^{n}\left\|\mathrm{~d} \theta\left(x_{i} \mid M_{*}, \Sigma_{*}, \pi_{*}\right)-\mathrm{d} \theta\left(x_{i} \mid M_{n}, \Sigma_{n}, \pi_{n}\right)\right\| \\
& \leq \frac{3}{n} \sum_{i=1}^{n}\left\|M_{*}^{-1} \Sigma_{*}\right\| \cdot\left\|g_{*}\left(x_{i}\right)-g_{n}\left(x_{i}\right)\right\|+\frac{3}{n} \sum_{i=1}^{n}\left\|M_{n}^{-1} \Sigma_{n}-M_{*}^{-1} \Sigma_{*}\right\| \cdot\left\|g_{n}\left(x_{i}\right)\right\|+3\left\|M_{n}^{-1}-M_{*}^{-1}\right\| \\
& =R_{1}+R_{2}+R_{3} .
\end{aligned}
$$

Here, by Proposition B.1, $\left\|\mathrm{d} \theta\left(x \mid M_{n}, \Sigma_{n}, \pi_{n}\right)\right\|$ and hence also $\left\|g_{n}(x)\right\|$ are uniformly bounded in $x$ for all large $n$. Then since $M_{n}, \Sigma_{n} \rightarrow M_{*}, \Sigma_{*}$, we have $R_{2}, R_{3} \rightarrow 0$. For $R_{1}$, fix $B>0$ and write

$$
\begin{aligned}
R_{1} & \leq \frac{C}{n} \sum_{i=1}^{n}\left\|g_{*}\left(x_{i}\right)-g_{n}\left(x_{i}\right)\right\| \cdot \mathbb{1}\left\{x_{i} \in \mathbb{B}^{k}(B)\right\}+\frac{C}{n} \sum_{i=1}^{n}\left(\left\|g_{*}\left(x_{i}\right)\right\|+\left\|g_{n}\left(x_{i}\right)\right\|\right) \mathbb{1}\left\{x_{i} \notin \mathbb{B}^{k}(B)\right\} \\
& \equiv(\mathrm{I})+(\mathrm{II}) .
\end{aligned}
$$

Applying the lower bounds $f_{n}(x), f_{*}(x) \geq c_{B}$ for all $x \in \mathbb{B}^{k}(B)$ and the uniform convergence of $f_{n}, \mathrm{~d} f_{n}$, and $\mathrm{d}^{2} f_{n}$ in Corollary B.5, we have $\lim _{n \rightarrow \infty}(\mathrm{I})=0$ for each fixed $B>0$. Then applying $\mathbf{X} \stackrel{W_{2}}{\rightarrow} X$ and the uniform boundedness of $\left\|g_{n}(x)\right\|$ and $\left\|g_{*}(x)\right\|$, we have $\lim _{B \rightarrow \infty} \limsup _{n \rightarrow \infty}$ (II) $=$ 0 . This shows $R_{1} \rightarrow 0$, concluding the proof.

Finally, we combine the above to prove Corollary 5.3 of the Main Text. Note that we have shown Lemma B.2 and its corollaries for any $\pi_{n}$ satisfying the condition (B.4). Then in particular, these results hold for the NPMLE $\pi_{n}=\operatorname{MLE}\left(\mathbf{X} \mid M_{n}, \Sigma_{n}, \mathcal{P}\right)$ defined by the maximization (2.8), and we record this implication here.

Corollary B.7. The conclusions of Lemma B.2, Corollary B.3, and Proposition B.6 hold for any $N P M L E \pi_{n}=\operatorname{MLE}\left(\mathbf{X} \mid M_{n}, \Sigma_{n}, \mathcal{P}\right)$ solving the maximization (2.8).

Proof. Applying the argument of Corollary B.5 with $\pi_{n}=\pi_{*}$, we have

$$
\sup _{x \in \mathbb{R}^{k}}\left|f_{\left(M_{n} \cdot \pi_{*}\right) * \mathcal{N}\left(0, \Sigma_{n}\right)}(x)-f_{\left(M_{*} \cdot \pi_{*}\right) * \mathcal{N}\left(0, \Sigma_{*}\right)}(x)\right| \rightarrow 0
$$

Fixing any $B>0$, since $f_{\left(M_{*} \cdot \pi_{*}\right) * \mathcal{N}\left(0, \Sigma_{*}\right)}(x)$ is bounded away from 0 for $x \in \mathbb{B}^{k}(B)$, this implies

$$
\frac{1}{n} \sum_{i=1}^{n}\left|\log \frac{f_{\left(M_{n} \cdot \pi_{*}\right) * \mathcal{N}\left(0, \Sigma_{n}\right)}\left(x_{i}\right)}{f_{\left(M_{*} \cdot \pi_{*}\right) * \mathcal{N}\left(0, \Sigma_{*}\right)}\left(x_{i}\right)}\right| \cdot \mathbb{1}\left\{x_{i} \in \mathbb{B}^{k}(B)\right\} \rightarrow 0 .
$$


To control this sum for $x_{i} \notin \mathbb{B}^{k}(B)$, note that for some constant $C>0$ independent of $n$, and for all $x \in \mathbb{R}^{k}$ and all large $n$,

$$
C \geq \log f_{\left(M_{n} \cdot \pi_{*}\right) * \mathcal{N}\left(0, \Sigma_{n}\right)}(x)=\log \mathbb{E}\left[\phi_{\Sigma_{n}}\left(x-M_{n} \Theta\right)\right] \geq \mathbb{E}\left[\log \phi_{\Sigma_{n}}\left(x-M_{n} \Theta\right)\right] \geq-C\left(1+\|x\|^{2}\right) .
$$

The same bounds hold for $\log f_{\left(M_{*} \cdot \pi_{*}\right) * \mathcal{N}\left(0, \Sigma_{*}\right)}(x)$. Hence

$$
\left|\log f_{\left(M_{*} \cdot \pi_{*}\right) * \mathcal{N}\left(0, \Sigma_{*}\right)}(x)\right|+\left|\log f_{\left(M_{n} \cdot \pi_{*}\right) * \mathcal{N}\left(0, \Sigma_{n}\right)}(x)\right| \leq C\left(1+\|x\|^{2}\right),
$$

so

$$
\frac{1}{n} \sum_{i=1}^{n}\left|\log \frac{f_{\left(M_{n} \cdot \pi_{*}\right) * \mathcal{N}\left(0, \Sigma_{n}\right)}\left(x_{i}\right)}{f_{\left(M_{*} \cdot \pi_{*}\right) * \mathcal{N}\left(0, \Sigma_{*}\right)}\left(x_{i}\right)}\right| \cdot \mathbb{1}\left\{x_{i} \notin \mathbb{B}^{k}(B)\right\} \leq \frac{2 C}{n} \sum_{i=1}^{n}\left(1+\left\|x_{i}\right\|^{2}\right) \mathbb{1}\left\{x_{i} \notin \mathbb{B}^{k}(B)\right\} .
$$

As shown for (B.8), this vanishes in the limit $n \rightarrow \infty$ followed by $B \rightarrow \infty$. Thus

$$
\frac{1}{n} \sum_{i=1}^{n} \log \frac{f_{\left(M_{n} \cdot \pi_{*}\right) * \mathcal{N}\left(0, \Sigma_{n}\right)}\left(x_{i}\right)}{f_{\left(M_{*} \cdot \pi_{*}\right) * \mathcal{N}\left(0, \Sigma_{*}\right)}\left(x_{i}\right)} \rightarrow 0 .
$$

Now letting $\pi_{n}=\operatorname{MLE}\left(\mathbf{X} \mid M_{n}, \Sigma_{n}, \mathcal{P}\right)$, by definition

$$
\frac{1}{n} \sum_{i=1}^{n} \log \frac{f_{\left(M_{n} \cdot \pi_{n}\right) * \mathcal{N}\left(0, \Sigma_{n}\right)}\left(x_{i}\right)}{f_{\left(M_{n} \cdot \pi_{*}\right) * \mathcal{N}\left(0, \Sigma_{n}\right)}\left(x_{i}\right)} \geq 0 .
$$

Combining the above two displays shows that $\pi_{n}$ satisfies the condition (B.4). Then Lemma B.2, Corollary B.3, and Proposition B.6 hold for $\pi_{n}$.

Proof of Corollary 5.3. By Proposition 5.2, we have $(\mathbf{U}, \mathbf{F}) \stackrel{W_{2}}{\rightarrow}(U, F)$ and $(\mathbf{V}, \mathbf{G}) \stackrel{W_{2}}{\rightarrow}(V, G)$. The convergence of $\sqrt{\gamma} \cdot \lambda_{i}$ in Lemma A.3 implies that $\hat{S}$ as defined in (2.14) is consistent for $S$. Then we have also the consistency of the plug-in estimators $\bar{M}, M, \bar{\Sigma}, \Sigma$ for $\bar{M}_{*}, M_{*}, \bar{\Sigma}_{*}, \Sigma_{*}$. Corollary 5.3 now follows directly from Corollary B.3, applied to the NPMLE $\pi_{n}$.

\section{State evolution of EB-PCA}

We prove Theorem 5.4 of the Main Text by comparing the EB-PCA trajectory with the trajectory of the oracle Bayes AMP iterates. Let $\mathbf{G}^{t}, \mathbf{V}^{t}, \mathbf{F}^{t}, \mathbf{U}^{t}$ denote the iterates of EB-PCA. We use ${ }^{\sim}$ to denote the analogous iterates of the oracle Bayes AMP algorithm. For example, corresponding to the EB-PCA iterates

$$
\begin{aligned}
& \mathbf{V}^{t}=\theta\left(\mathbf{G}^{t} \mid M_{t}, \Sigma_{t}, \pi_{t}\right) \\
& \mathbf{F}^{t}=\mathbf{Y} \mathbf{V}^{t}-\mathbf{U}^{t-1} \cdot \gamma B_{t}^{\top} \quad \text { where } B_{t}=\left\langle\mathbf{d} \theta\left(\mathbf{G}^{t} \mid M_{t}, \Sigma_{t}, \pi_{t}\right)\right\rangle,
\end{aligned}
$$

we have the oracle Bayes AMP iterates

$$
\begin{aligned}
& \tilde{\mathbf{V}}^{t}=\theta\left(\tilde{\mathbf{G}}^{t} \mid M_{*, t}, \Sigma_{*, t}, \pi_{*}\right) \\
& \tilde{\mathbf{F}}^{t}=\mathbf{Y} \tilde{\mathbf{V}}^{t}-\tilde{\mathbf{U}}^{t-1} \cdot \gamma \tilde{B}_{t}^{\top} \quad \text { where } \tilde{B}_{t}=\left\langle\mathrm{d} \theta\left(\tilde{\mathbf{G}}^{t} \mid M_{*, t}, \Sigma_{*, t}, \pi_{*}\right)\right\rangle .
\end{aligned}
$$

This oracle Bayes AMP algorithm is initialized with $\tilde{\mathbf{G}}^{0}=\mathbf{G}, \tilde{\mathbf{U}}^{-1}=\mathbf{F} \cdot \Sigma_{*, 0}^{1 / 2}$, and $M_{*, 0}=M_{*}$ and $\Sigma_{*, 0}=\Sigma_{*}$ as described in (2.11) and (2.12). The matrices $\left\{\bar{M}_{*, t}, \bar{\Sigma}_{*, t}\right\}$ and $\left\{M_{*, t+1}, \Sigma_{*, t+1}\right\}$ are defined iteratively by the true state evolution $((2.19))$, where $u_{t}(\cdot)$ and $v_{t}(\cdot)$ are the true posterior mean denoisers defined by $(2.20)$. 


\section{C.1 State evolution of oracle Bayes AMP}

The validity of the state evolution for this oracle Bayes AMP procedure was shown in [MV21].

Theorem C.1. Consider the rank-k signal-plus-noise model of (2.1), and suppose that Assumption 5.1 holds. Then for each fixed iterate $t$, as $n, d \rightarrow \infty$, almost surely

$$
\left(\mathbf{U}, \tilde{\mathbf{F}}^{t}\right) \stackrel{W_{2}}{\rightarrow}\left(U, F_{t}\right) \quad \text { and } \quad\left(\mathbf{V}, \tilde{\mathbf{G}}^{t}\right) \stackrel{W_{2}}{\rightarrow}\left(V, G_{t}\right),
$$

where $U \sim \bar{\pi}_{*}, F_{t} \mid U \sim \mathcal{N}\left(\bar{M}_{*, t} \cdot U, \bar{\Sigma}_{*, t}\right), V \sim \pi_{*}$, and $G_{t} \mid V \sim \mathcal{N}\left(M_{*, t} \cdot V, \Sigma_{*, t}\right)$.

Proof. This follows from [MV21, Theorem 7], specializing to the setting where all $k$ signal values $s_{1}, \ldots, s_{k}$ are distinct and super-critical.

We elaborate on the oracle Bayes initialization

$$
\mathbf{U}^{-1}=\mathbf{F} \cdot \Sigma_{*}^{1 / 2}
$$

discussed in Remark 2.4 of the Main Text, corresponding to $\mathbf{F} \cdot \Sigma_{0}^{1 / 2}$ in Algorithm 1. Informally, initializing AMP with the sample PCs $\mathbf{G}$ may be understood as first applying a large number of iterations of a linear AMP iteration whose fixed points are approximately the sample PCs in the $n, d \rightarrow \infty$ limit, and then transitioning the algorithm to apply the non-linear oracle Bayes denoisers. This linear AMP algorithm applies the functions $u_{t, \text { lin }}(\mathbf{F})=\mathbf{F} \cdot D$ and $v_{t, \text { lin }}(\mathbf{G})=\mathbf{G} \cdot E$ for two fixed diagonal matrices $D, E \in \mathbb{R}^{k \times k}$. Then $\left\langle\mathrm{d} u_{t, \text { lin }}(\mathbf{F})\right\rangle^{\top}=D$ and $\left\langle\mathbf{d} v_{t, \operatorname{lin}}(\mathbf{G})\right\rangle^{\top}=E$. For $\mathbf{F}, \mathbf{G}$ to be approximate fixed points of the linear AMP iterations, we require

$$
\begin{aligned}
& \mathbf{F} \approx \mathbf{Y} v_{t, \operatorname{lin}}(\mathbf{G})-u_{t-1, \operatorname{lin}}(\mathbf{F}) \cdot \gamma E=\gamma \mathbf{F} \Lambda E-\gamma \mathbf{F} D E \\
& \mathbf{G} \approx \mathbf{Y}^{\top} u_{t, \operatorname{lin}}(\mathbf{F})-v_{t, \operatorname{lin}}(\mathbf{G}) \cdot D=\mathbf{G} \Lambda D-\mathbf{G} E D .
\end{aligned}
$$

Thus $D, E$ should satisfy $\operatorname{Id} \approx \gamma(\Lambda-D) E$ and $\operatorname{Id} \approx(\Lambda-E) D$. Here $\Lambda$ is random, but by $(2.10)$ converges to $\Sigma_{*}^{1 / 2} \cdot\left(S^{2}+\mathrm{Id}\right)$ for large $n, d$. Thus we choose $D, E$ to solve the pair of equations

$$
\mathrm{Id}=\gamma\left(\Sigma_{*}^{1 / 2} \cdot\left(S^{2}+\mathrm{Id}\right)-D\right) E, \quad \operatorname{Id}=\left(\Sigma_{*}^{1 / 2} \cdot\left(S^{2}+\mathrm{Id}\right)-E\right) D
$$

yielding

$$
D=\Sigma_{*}^{1 / 2}, \quad E=\gamma^{-1 / 2} \bar{\Sigma}_{*}^{1 / 2}
$$

where $\Sigma_{*}, \bar{\Sigma}_{*}$ are as described in (2.12). Then corresponding to the PCA initialization $\mathbf{G}^{0}=\mathbf{G}$, we should set

$$
\mathbf{U}^{-1}=u_{t, \operatorname{lin}}(\mathbf{F})=\mathbf{F} \cdot \Sigma_{*}^{1 / 2} \text {. }
$$

More formally, the analysis of [MV21] shows that the state evolution for the iterates of the oracle Bayes AMP procedure initialized at $\mathbf{G}^{0}=\mathbf{G}$ coincides with that of an AMP procedure using the matrix

$$
\tilde{\mathbf{Y}}=\frac{1}{n} \mathbf{F} \Lambda \mathbf{G}^{\top}+\mathbf{P}_{\mathbf{F}}^{\perp}\left(\frac{1}{n} \mathbf{U} S \mathbf{V}^{\top}+\tilde{\mathbf{W}}\right) \mathbf{P}_{\mathbf{G}}^{\perp},
$$

where $\mathbf{P}_{\overline{\mathbf{F}}}^{\perp}$ and $\mathbf{P}_{\mathbf{G}}^{\perp}$ are the projections orthogonal to the column spans of $\mathbf{F}$ and $\mathbf{G}$, and $\tilde{\mathbf{W}}$ is a copy of $\mathbf{W}$ independent of all other quantities. Following the calculations in [MV21], it may be verified that setting $\mathbf{U}^{-1}=\mathbf{F} \cdot \Sigma_{*}^{1 / 2}$ rather than $\mathbf{U}^{-1}=0$ is required for the state evolution to be correct for describing

$$
\mathbf{F}^{0}=\tilde{\mathbf{Y}} v_{0}(\mathbf{G})-\mathbf{U}^{-1} \cdot \gamma\left\langle v_{0}(\mathbf{G})\right\rangle
$$




\section{C.2 Comparison with oracle Bayes AMP}

Proposition C.2. Under Assumption 5.1, for all t, the matrices $\Sigma_{*, t}, \bar{\Sigma}_{*, t} \in \mathbb{R}^{k \times k}$ are invertible.

Proof. The initialization $\Sigma_{*, 0}$ is a diagonal matrix with non-zero diagonal entries, thus invertible. We will show, if $\Sigma_{*, t}$ is invertible, then $\bar{\Sigma}_{*, t}$ is also invertible.

Suppose by contradiction that for some non-zero $\phi \in \mathbb{R}^{k}$,

$$
0=\phi^{\top} \bar{\Sigma}_{*, t} \phi=\gamma \cdot \mathbb{E}\left[\mathbb{E}\left[\phi^{\top} V \mid M_{*, t} V+\Sigma_{*, t}^{1 / 2} Z\right]^{2}\right] .
$$

Note that by $(2.21), M_{*, t}$ must be invertible if $\Sigma_{*, t}$ is invertible. Then almost surely with respect to the distribution of $V+M_{*, t}^{-1} \Sigma_{*, t}^{1 / 2} Z$, we must have $\mathbb{E}\left[\phi^{\top} V \mid V+M_{*, t}^{-1} \Sigma_{*, t}^{1 / 2} Z\right]=0$. Denote $\Omega=\operatorname{Cov}\left[M_{*, t}^{-1} \Sigma_{*, t}^{1 / 2} Z\right]^{-1}=\Sigma_{*, t}^{-1 / 2} M_{*, t}^{2} \Sigma_{*, t}^{-1 / 2}$. Since $V+M_{*, t}^{-1} \Sigma_{*, t}^{1 / 2} Z$ is supported on all of $\mathbb{R}^{k}$, this implies that the (continuous) function

$$
f(x)=\mathbb{E}\left[\phi^{\top} V \mid V+M_{*, t}^{-1} \Sigma_{*, t}^{1 / 2} Z=x\right]=\frac{\mathbb{E}\left[\phi^{\top} V \cdot \exp \left(-\frac{1}{2} V^{\top} \Omega V+x^{\top} \Omega V\right)\right]}{\mathbb{E}\left[\exp \left(-\frac{1}{2} V^{\top} \Omega V+x^{\top} \Omega V\right)\right]}
$$

must be identically 0 for all $x \in \mathbb{R}^{k}$. Then the gradient of its numerator in $x$,

$$
g(x)=\mathbb{E}\left[\Omega V \cdot \phi^{\top} V \cdot \exp \left(-\frac{1}{2} V^{\top} \Omega V+x^{\top} \Omega V\right)\right],
$$

is also identically 0 for all $x \in \mathbb{R}^{k}$, so

$$
0=\phi^{\top} \Omega^{-1} g(x)=\mathbb{E}\left[\left(\phi^{\top} V\right)^{2} \exp \left(-\frac{1}{2} V^{\top} \Omega V+x^{\top} \Omega V\right)\right] .
$$

Then we must have $\phi^{\top} V=0$ almost surely. However, by assumption $\mathbb{E}\left[\phi^{\top} V V^{\top} \phi\right]=\phi^{\top} \operatorname{Id}_{k \times k} \phi=$ $\|\phi\|^{2} \neq 0$, a contradiction. So $\bar{\Sigma}_{*, t}$ is invertible.

If $\bar{\Sigma}_{*, t}$ is invertible, an analogous argument shows $\Sigma_{*, t+1}$ is invertible, concluding the proof.

Proof of Theorem 5.4. Let $H_{t}$ be the hypothesis that the following hold almost surely as $n, d \rightarrow \infty$ :

1. $\left(M_{t}, \Sigma_{t}\right) \rightarrow\left(M_{*, t}, \Sigma_{*, t}\right)$,

2. $\frac{1}{n}\left\|\mathbf{U}^{t-1}-\tilde{\mathbf{U}}^{t-1}\right\|_{F}^{2} \rightarrow 0$,

3. $\frac{1}{d}\left\|\mathbf{G}^{t}-\tilde{\mathbf{G}}^{t}\right\|_{F}^{2} \rightarrow 0$.

Similarly, let $\bar{H}_{t}$ be the hypothesis that the following hold almost surely as $n, d \rightarrow \infty$ :

1. $\left(\bar{M}_{t}, \bar{\Sigma}_{t}\right) \rightarrow\left(\bar{M}_{*, t}, \bar{\Sigma}_{*, t}\right)$,

2. $\frac{1}{d}\left\|\mathbf{V}^{t}-\tilde{\mathbf{V}}^{t}\right\|_{F}^{2} \rightarrow 0$,

3. $\frac{1}{n}\left\|\mathbf{F}^{t}-\tilde{\mathbf{F}}^{t}\right\|_{F}^{2} \rightarrow 0$.

Note that by Theorem C.1, $\left(\mathbf{V}, \tilde{\mathbf{G}}^{t}\right) \stackrel{W_{2}}{\rightarrow}\left(V, G_{t}\right)$. Then $H_{t} .3$ implies $\left(\mathbf{V}, \mathbf{G}^{t}\right) \stackrel{W_{2}}{\rightarrow}\left(V, G_{t}\right)$. Similarly, $\bar{H}_{t} .3$ implies $\left(\mathbf{U}, \mathbf{F}^{t}\right) \stackrel{W_{2}}{\rightarrow}\left(U, F_{t}\right)$, which establishes Theorem 5.4.

To complete the proof, we show that $H_{0}$ holds, that $H_{t}$ implies $\bar{H}_{t}$, and that $\bar{H}_{t}$ implies $H_{t+1}$. 
Step 1: $H_{0}$ holds. Lemma A.3 implies that the estimates $\hat{S}, M_{0}$, and $\Sigma_{0}$ in Algorithm 1 are consistent for $S, M_{*, 0}$, and $\Sigma_{*, 0}$ as $n, d \rightarrow \infty$. Then $H_{0} .1$ holds. $H_{0} .3$ holds trivially because $\mathbf{G}^{0}=\tilde{\mathbf{G}}^{0}$, as the two algorithms have the same initialization. $H_{0} .2$ also holds, because

$$
\frac{1}{n}\left\|\mathbf{U}^{-1}-\tilde{\mathbf{U}}^{-1}\right\|_{F}^{2} \leq \frac{1}{n}\|\mathbf{F}\|_{F}^{2} \cdot\left\|\Sigma_{0}-\Sigma_{*, 0}\right\| \rightarrow 0
$$

by the consistency of $\Sigma_{0}$ for $\Sigma_{*, 0}$ and the normalization $n^{-1}\|\mathbf{F}\|_{F}^{2}=k$.

Step 2: $H_{t} \Rightarrow \bar{H}_{t} .2$. Let $g_{j}^{t}$ and $\tilde{g}_{j}^{t}$ denote the rows of $\mathbf{G}^{t}$ and $\tilde{\mathbf{G}}^{t}$. Then

$$
\begin{aligned}
\frac{1}{d}\left\|\mathbf{V}^{t}-\tilde{\mathbf{V}}^{t}\right\|_{F}^{2} & =\frac{1}{d} \sum_{j=1}^{d}\left\|\theta\left(\tilde{g}_{j}^{t} \mid M_{*, t}, \Sigma_{*, t}, \pi_{*}\right)-\theta\left(g_{j}^{t} \mid M_{t}, \Sigma_{t}, \pi_{t}\right)\right\|^{2} \\
\leq & \frac{2}{d} \sum_{j=1}^{d}\left\|\theta\left(g_{j}^{t} \mid M_{*, t}, \Sigma_{*, t}, \pi_{*}\right)-\theta\left(g_{j}^{t} \mid M_{t}, \Sigma_{t}, \pi_{t}\right)\right\|^{2} \\
& +\frac{2}{d} \sum_{j=1}^{d}\left\|\theta\left(\tilde{g}_{j}^{t} \mid M_{*, t}, \Sigma_{*, t}, \pi_{*}\right)-\theta\left(g_{j}^{t} \mid M_{*, t}, \Sigma_{*, t}, \pi_{*}\right)\right\|^{2} .
\end{aligned}
$$

By Corollary B.3, (I) $\stackrel{\text { a.s. }}{\rightarrow}$ 0. By Assumption 5.1, $\theta\left(\cdot \mid M_{*, t}, \Sigma_{*, t}, \pi_{*}\right)$ is Lipschitz continuous with some Lipschitz constant $L$. Applying this and $H_{t} .3$,

$$
(\mathrm{II}) \leq \frac{2 L^{2}}{d} \sum_{j=1}^{d}\left\|\tilde{g}_{j}^{t}-g_{j}^{t}\right\|^{2}=2 L^{2} \cdot \frac{1}{d}\left\|\tilde{\mathbf{G}}^{t}-\mathbf{G}^{t}\right\|_{F}^{2} \stackrel{\text { a.s. }}{\rightarrow} 0 .
$$

Step 3: $H_{t} \Rightarrow \bar{H}_{t} .3$. Recalling (C.1) and (C.2),

$$
\begin{aligned}
\frac{1}{\sqrt{n}}\left\|\mathbf{F}^{t}-\tilde{\mathbf{F}}^{t}\right\|_{F} & \leq\|\mathbf{Y}\| \cdot \frac{1}{\sqrt{n}}\left\|\mathbf{V}^{t}-\tilde{\mathbf{V}}^{t}\right\|_{F}+\left\|B_{t}-\tilde{B}_{t}\right\| \cdot \frac{\gamma}{\sqrt{n}}\left\|\tilde{\mathbf{U}}^{t-1}\right\|_{F}+\left\|B_{t}\right\| \cdot \frac{\gamma}{\sqrt{n}}\left\|\tilde{\mathbf{U}}^{t-1}-\mathbf{U}^{t-1}\right\|_{F} \\
& =R_{1}+R_{2}+R_{3} .
\end{aligned}
$$

Since $\|\mathbf{Y}\|$ converges to a constant almost surely, and $n^{-1}\left\|\mathbf{V}^{t}-\tilde{\mathbf{V}}^{t}\right\|_{F}^{2} \stackrel{\text { a.s. }}{\rightarrow} 0$, we have $R_{1} \stackrel{\text { a.s. }}{\rightarrow} 0$. To show $R_{2} \stackrel{\text { a.s. }}{\rightarrow} 0$, note that $n^{-1}\left\|\tilde{\mathbf{U}}^{t-1}\right\|_{F}^{2}$ converges to a constant almost surely by Theorem C.1, so we only need to show $\left\|B_{t}-\tilde{B}_{t}\right\| \stackrel{\text { a.s. }}{\rightarrow} 0$. Let us write

$$
\begin{aligned}
\left\|B_{t}-\tilde{B}_{t}\right\| & =\left\|\frac{1}{d} \sum_{j=1}^{d} \mathrm{~d} \theta\left(\tilde{g}_{j}^{t} \mid M_{*, t}, \Sigma_{*, t}, \pi_{*}\right)-\frac{1}{d} \sum_{j=1}^{d} \mathrm{~d} \theta\left(g_{j}^{t} \mid M_{t}, \Sigma_{t}, \pi_{t}\right)\right\| \\
\leq & \frac{1}{d} \sum_{j=1}^{d}\left\|\mathrm{~d} \theta\left(g_{j}^{t} \mid M_{*, t}, \Sigma_{*, t}, \pi_{*}\right)-\mathrm{d} \theta\left(g_{j}^{t} \mid M_{t}, \Sigma_{t}, \pi_{t}\right)\right\| \\
& +\frac{1}{d} \sum_{j=1}^{d}\left\|\mathrm{~d} \theta\left(\tilde{g}_{j}^{t} \mid M_{*, t}, \Sigma_{*, t}, \pi_{*}\right)-\mathrm{d} \theta\left(g_{j}^{t} \mid M_{*, t}, \Sigma_{*, t}, \pi_{*}\right)\right\|
\end{aligned}
$$


By Proposition B.6, (I) $\stackrel{\text { a.s. }}{\rightarrow} 0$. To show $(\mathrm{II}) \stackrel{\text { a.s. }}{\rightarrow} 0$, note that the Lipschitz assumption implies $\left\|\mathrm{d} \theta\left(\cdot \mid M_{*, t}, \Sigma_{*, t}, \pi_{*}\right)\right\| \leq L$. Then for any fixed $B>0$,

$$
\begin{gathered}
(\mathrm{II}) \leq \frac{1}{d} \sum_{j=1}^{d}\left\|\mathrm{~d} \theta\left(\tilde{g}_{j}^{t} \mid M_{*, t}, \Sigma_{*, t}, \pi_{*}\right)-\mathrm{d} \theta\left(g_{j}^{t} \mid M_{*, t}, \Sigma_{*, t}, \pi_{*}\right)\right\| \cdot \mathbb{1}\left\{g_{j}^{t}, \tilde{g}_{j}^{t} \in \mathbb{B}^{k}(B)\right\} \\
+\frac{2 L}{d} \sum_{j=1}^{d} \mathbb{1}\left\{g_{j}^{t} \notin \mathbb{B}^{k}(B)\right\}+\frac{2 L}{d} \sum_{j=1}^{d} \mathbb{1}\left\{\tilde{g}_{j}^{t} \notin \mathbb{B}^{k}(B)\right\} .
\end{gathered}
$$

The last two terms are at most

$$
\frac{2 L}{d B^{2}}\left(\left\|\mathbf{G}^{t}\right\|_{F}^{2}+\left\|\tilde{\mathbf{G}}^{t}\right\|_{F}^{2}\right)
$$

To control the first term, observe that $f(x)>c$ and $\left\|\mathrm{d}^{i} f(x)\right\|<C$ for all $x \in \mathbb{B}^{k}(B)$, all $i=1,2,3$, and some constants $C, c>0$ (depending on $B$ ). Then differentiating (B.10) again in $x$ yields $\left\|\mathrm{d}^{2} \theta\left(x \mid M_{*, t}, \Sigma_{*, t}, \pi_{*}\right)\right\|<C_{B}$ for a constant $C_{B}>0$ and all $x \in \mathbb{B}^{k}(B)$. Thus the first term is at most

$$
\frac{C_{B}}{d} \sum_{j=1}^{d}\left\|\tilde{g}_{j}^{t}-g_{j}^{t}\right\| \leq \frac{C_{B}}{\sqrt{d}}\left\|\tilde{\mathbf{G}}^{t}-\mathbf{G}^{t}\right\|_{F} .
$$

Applying $H_{t} .3$ and taking first the limit $n, d \rightarrow \infty$, followed by the limit $B \rightarrow \infty$, we have (II) $\stackrel{\text { a.s. }}{\rightarrow} 0$, and thus $\left\|B_{t}-\tilde{B}_{t}\right\| \stackrel{\text { a.s. }}{\rightarrow} 0$. This shows $R_{2} \stackrel{\text { a.s. }}{\rightarrow} 0$. This further implies $\lim \sup _{n}\left\|B_{t}\right\|<\infty$ because Theorem C.1 guarantees that $\lim _{\sup _{n}}\left\|\tilde{B}_{t}\right\|<\infty$. Combining this with $H_{t} .2$ shows $R_{3} \stackrel{\text { a.s. }}{\rightarrow} 0$.

Step 4: $H_{t} \Rightarrow \bar{H}_{t} .1$. As $\theta\left(\cdot \mid M_{*, t}, \Sigma_{*, t}, \pi_{t}\right) \otimes \theta\left(\cdot \mid M_{*, t}, \Sigma_{*, t}, \pi_{t}\right) \in \operatorname{PL}(2)$, Theorem C.1 implies

$$
\bar{\Sigma}_{*, t}=\lim _{n, d \rightarrow \infty} \frac{\gamma}{d} \sum_{j=1}^{d} \tilde{v}_{j}^{t} \otimes \tilde{v}_{j}^{t}=\left(\tilde{\mathbf{V}}^{t}\right)^{\top} \tilde{\mathbf{V}}^{t} / n .
$$

Applying the definition of $\bar{\Sigma}_{t}$ in Algorithm 1, together with $\bar{H}_{t} .2$ already shown and CauchySchwarz, this limit is the same as

$$
\lim _{n, d \rightarrow \infty} \bar{\Sigma}_{t}=\lim _{n, d \rightarrow \infty}\left(\mathbf{V}^{t}\right)^{\top} \mathbf{V}^{t} / n
$$

so $\bar{\Sigma}_{t} \rightarrow \bar{\Sigma}_{*, t}$. Recalling (2.21), $\bar{M}_{t}=\bar{\Sigma}_{t} \cdot \hat{S}$, and applying the consistency of $\hat{S}$, this implies also $\bar{M}_{t} \rightarrow \bar{M}_{*, t}$.

This completes the proof that $H_{t}$ implies $\bar{H}_{t}$. The proof that $\bar{H}_{t}$ implies $H_{t+1}$ is the same as steps $2-4$ above.

\section{Analysis of the limiting risk}

\section{D.1 Reparametrization of the states}

Recall from Section 5.3 of the Main Text the definitions

$$
\bar{Q}_{*, t}=\frac{1}{\gamma} S^{-1 / 2} \bar{M}_{*, t}^{\top} \bar{\Sigma}_{*, t}^{-1} \bar{M}_{*, t} S^{-1 / 2}, \quad Q_{*, t}=S^{-1 / 2} M_{*, t}^{\top} \Sigma_{*, t}^{-1} M_{*, t} S^{-1 / 2}
$$


and the map

$$
F_{\pi}(Q)=\mathbb{E}\left[\mathbb{E}\left[\Theta \mid \Theta+Q^{-1 / 2} Z\right]^{\otimes 2}\right], \quad \Theta \sim \pi, Z \sim \mathcal{N}(0, \text { Id }) \text { independent. }
$$

For each $\bar{Q}_{*, t}$ and $Q_{*, t+1}$ with $t \geq 0$, recalling the identities $\bar{M}_{*, t}=\bar{\Sigma}_{*, t} \cdot S$ and $M_{*, t+1}=\Sigma_{*, t+1} \cdot S$ from (2.21), we have

$$
\bar{Q}_{*, t}=\frac{1}{\gamma} S^{1 / 2} \bar{\Sigma}_{*, t} S^{1 / 2}, \quad Q_{*, t+1}=S^{1 / 2} \Sigma_{*, t+1} S^{1 / 2}
$$

Then substituting from (2.19) the definition

$$
\begin{aligned}
\bar{\Sigma}_{*, t} & =\gamma \cdot \mathbb{E}\left[\mathbb{E}\left[V \mid M_{*, t} V+\Sigma_{*, t}^{1 / 2} Z\right]^{\otimes 2}\right] \\
& =\gamma \cdot \mathbb{E}\left[\mathbb{E}\left[V \mid S^{1 / 2} V+S^{1 / 2} M_{*, t}^{-1} \Sigma_{*, t}^{1 / 2} Z\right]^{\otimes 2}\right]=\gamma \cdot \mathbb{E}\left[\mathbb{E}\left[V \mid S^{1 / 2} V+Q_{*, t}^{-1 / 2} Z\right]^{\otimes 2}\right],
\end{aligned}
$$

we obtain

$$
\bar{Q}_{*, t}=\frac{1}{\gamma} S^{1 / 2} \bar{\Sigma}_{*, t} S^{1 / 2}=\mathbb{E}\left[\mathbb{E}\left[S^{1 / 2} V \mid S^{1 / 2} V+Q_{*, t}^{-1 / 2} Z\right]^{\otimes_{2}}\right]=F_{S^{1 / 2} \pi_{*}}\left(Q_{*, t}\right) .
$$

Similarly,

$$
Q_{*, t+1}=S^{1 / 2} \Sigma_{*, t+1} S^{1 / 2}=\mathbb{E}\left[\mathbb{E}\left[S^{1 / 2} U \mid S^{1 / 2} U+\left(\gamma \bar{Q}_{*, t}\right)^{-1 / 2} Z\right]^{\otimes_{2}}\right]=F_{S^{1 / 2} \bar{\pi}_{*}}\left(\gamma \cdot \bar{Q}_{*, t}\right) .
$$

This verifies the equivalent forms of (5.2) for the state evolution as stated in the Main Text.

\section{D.2 Progression of SNR}

We now prove Proposition 5.6 of the Main Text. In the standardized compound decision model

$$
\Theta \sim \pi, \quad X \mid \Theta \sim \mathcal{N}\left(0, Q^{-1}\right)
$$

parametrized by $Q$ and $\pi$, let us define

$$
M_{\pi}(Q)=\mathbb{E}[\operatorname{Cov}[\Theta \mid X]]=\mathbb{E}\left[(\Theta-\mathbb{E}[\Theta \mid X])^{\otimes 2}\right]
$$

so that

$$
F_{\pi}(Q)=\mathbb{E}\left[\Theta \Theta^{\top}\right]-M_{\pi}(Q)
$$

We will use the following properties of $F_{\pi}(Q)$ and $M_{\pi}(Q)$ established in [Mio17] and [RPD18].

Lemma D.1 (Lemma 9 in [Mio17]). If $Q_{1} \preceq Q_{2}$, then $F_{\pi}\left(Q_{1}\right) \preceq F_{\pi}\left(Q_{2}\right)$.

Lemma D.2 (Theorem 2 in $[\operatorname{RPD} 18]) \cdot M_{\pi}(Q) \preceq\left(\operatorname{Cov}[\Theta]^{-1}+Q\right)^{-1}$.

Proof of Proposition 5.6. For part (a), observe that by (2.10), $Q_{*, 0}$ is the diagonal matrix

$$
Q_{*, 0}=\operatorname{diag}\left(\frac{1}{s_{i}} \cdot \frac{\gamma s_{i}^{4}-1}{\gamma s_{i}^{2}+1}\right)
$$


Furthermore, applying (D.1), Lemma D.2, and $\mathbb{E}\left[V V^{\top}\right]=\mathbb{E}\left[U U^{\top}\right]=\mathrm{Id}$,

$$
\begin{aligned}
\bar{Q}_{*, 0}=F_{S^{1 / 2} \cdot \pi_{*}}\left(Q_{0}\right) & =\mathbb{E}\left[S^{1 / 2} V V^{\top} S^{1 / 2}\right]-M_{S^{1 / 2} \cdot \pi_{*}}\left(Q_{*, 0}\right) \\
& \succeq \mathbb{E}\left[S^{1 / 2} V V^{\top} S^{1 / 2}\right]-\left(\mathbb{E}\left[S^{1 / 2} V V^{\top} S^{1 / 2}\right]^{-1}+Q_{*, 0}\right)^{-1} \\
& =S-\left(S^{-1}+Q_{0}\right)^{-1}=\operatorname{diag}\left(\frac{\gamma s_{i}^{4}-1}{\gamma s_{i}\left(1+s_{i}^{2}\right)}\right), \\
Q_{1}=F_{S^{1 / 2} \cdot \bar{\pi}_{*}}\left(\gamma \bar{Q}_{*, 0}\right) & =\mathbb{E}\left[S^{1 / 2} U U^{\top} S^{1 / 2}\right]-M_{S^{1 / 2} \cdot \bar{\pi}_{*}}\left(\gamma \bar{Q}_{*, 0}\right) \\
& \succeq \mathbb{E}\left[S^{1 / 2} U U^{\top} S^{1 / 2}\right]-\left(\mathbb{E}\left[S^{1 / 2} U U^{\top} S^{1 / 2}\right]^{-1}+\gamma \bar{Q}_{*, 0}\right)^{-1} \\
& =S-\left(S^{-1}+\operatorname{diag}\left(\frac{\gamma s_{i}^{4}-1}{s_{i}\left(1+s_{i}^{2}\right)}\right)\right)^{-1}=Q_{*, 0} .
\end{aligned}
$$

Thus $Q_{*, 1} \succeq Q_{*, 0}$. Then $\bar{Q}_{*, 1} \succeq \bar{Q}_{*, 0}, Q_{*, 2} \succeq Q_{*, 1}$, etc. by (5.2) and the monotonicity of $F_{\pi}$ established in Lemma D.1. Since $\bar{Q}_{*, t} \preceq \mathbb{E}\left[S^{1 / 2} V V^{\top} S^{1 / 2}\right]=S$ and similarly $Q_{*, t} \preceq S$, this implies that $\left\{\bar{Q}_{*, t}\right\}_{t \geq 0}$ and $\left\{Q_{*, t}\right\}_{t \geq 0}$ must converge to some limits $\bar{Q}$ and $Q$, which must satisfy (5.3). This shows part (a).

For part (b), in the compound decision model $X=M \Theta+\Sigma^{1 / 2} Z$ where $\Theta \sim \pi$ and $Z \sim \mathcal{N}(0$, Id), set $Q=\Sigma^{-1 / 2} M S^{-1 / 2}$ and note that

$$
\begin{aligned}
\operatorname{mmse}(\pi \mid M, \Sigma) & =\mathbb{E}\left[\left\|\Theta-\mathbb{E}\left[\Theta \mid M \Theta+\Sigma^{1 / 2} Z\right]\right\|^{2}\right] \\
& =\operatorname{Tr} \mathbb{E}\left[\operatorname{Cov}\left[\Theta \mid M \Theta+\Sigma^{1 / 2} Z\right]\right] \\
& =\operatorname{Tr} \mathbb{E}\left[\operatorname{Cov}\left[\Theta \mid S^{1 / 2} \Theta+S^{1 / 2} M^{-1} \Sigma^{1 / 2} Z\right]\right] \\
& =\operatorname{Tr} S^{-1 / 2} \mathbb{E}\left[\operatorname{Cov}\left[S^{1 / 2} \Theta \mid S^{1 / 2} \Theta+Q^{-1 / 2} Z\right]\right] S^{-1 / 2} \\
& =\operatorname{Tr} S^{-1 / 2} M_{S^{1 / 2} \cdot \pi}(Q) S^{-1 / 2} .
\end{aligned}
$$

The relation of (D.1) and part (a) imply $M_{S^{1 / 2} \cdot \pi_{*}}\left(Q_{*, t+1}\right) \preceq M_{S^{1 / 2} \cdot \pi_{*}}\left(Q_{*, t}\right)$, so

$$
\begin{aligned}
\operatorname{mmse}\left(\pi_{*} \mid M_{*, t+1}, \Sigma_{*, t+1}\right) & =\operatorname{Tr} S^{-1 / 2} M_{S^{1 / 2} \cdot \pi_{*}}\left(Q_{*, t+1}\right) S^{-1 / 2} \\
& \leq \operatorname{Tr} S^{-1 / 2} M_{S^{1 / 2} \cdot \pi_{*}}\left(Q_{*, t}\right) S^{-1 / 2}=\operatorname{mmse}\left(\pi_{*} \mid M_{*, t}, \Sigma_{*, t}\right) .
\end{aligned}
$$

Similarly $\operatorname{mmse}\left(\bar{\pi}_{*} \mid \bar{M}_{*, t+1}, \bar{\Sigma}_{*, t+1}\right) \leq \operatorname{mmse}\left(\bar{\pi}_{*} \mid \bar{M}_{*, t}, \bar{\Sigma}_{*, t}\right)$. Since $\mathbf{V}^{0}=\hat{\mathbf{V}}$ is exactly the initial empirical Bayes estimate for $\mathbf{V}$, we have $\operatorname{mmse}\left(\pi_{*} \mid M_{*, 0}, \Sigma_{*, 0}\right)=\operatorname{mmse}\left(\pi_{*} \mid M_{*}, \Sigma_{*}\right)$. For $\mathbf{U}$, we observe that the signal-to-noise matrix analogous to $Q_{*, 0}$ defined by $\bar{M}_{*}$ and $\bar{\Sigma}_{*}$ for the left sample PCs is, from (2.10),

$$
\frac{1}{\gamma} S^{-1 / 2} \bar{M}_{*}^{\top} \bar{\Sigma}_{*}^{-1} \bar{M}_{*} S^{-1 / 2}=\operatorname{diag}\left(\frac{1}{\gamma s_{i}} \cdot \frac{\gamma s_{i}^{4}-1}{s_{i}^{2}+1}\right) .
$$

This is exactly the lower bound established above for $\bar{Q}_{*, 0}$, so we also have mmse $\left(\bar{\pi}_{*} \mid \bar{M}_{*, 0}, \bar{\Sigma}_{*, 0}\right) \leq$ $\operatorname{mmse}\left(\pi_{*} \mid \bar{M}_{*}, \bar{\Sigma}_{*}\right)$. This shows part (b).

\section{D.3 Bayes optimality}

Proof of Proposition 5.7. Expanding the square, we have

$$
\left\|\mathbf{U}^{t} \hat{S}\left(\mathbf{V}^{t}\right)^{\top}-\mathbf{U} S \mathbf{V}^{\top}\right\|_{F}^{2}=\operatorname{Tr}\left(\mathbf{U}^{t}\right)^{\top} \mathbf{U}^{t} \hat{S}\left(\mathbf{V}^{t}\right)^{\top} \mathbf{V}^{t} \hat{S}-2 \operatorname{Tr} \mathbf{U}^{\top} \mathbf{U}^{t} \hat{S}\left(\mathbf{V}^{t}\right)^{\top} \mathbf{V} S+\operatorname{Tr} \mathbf{U}^{\top} \mathbf{U} S \mathbf{V}^{\top} \mathbf{V} S
$$

Theorem 5.4 implies

$$
n^{-1}\left(\mathbf{U}^{t}\right)^{\top} \mathbf{U}^{t} \rightarrow \mathbb{E}\left[U_{t} U_{t}^{\top}\right], \quad n^{-1}\left(\mathbf{U}^{t}\right)^{\top} \mathbf{U} \rightarrow \mathbb{E}\left[U_{t} U^{\top}\right]=\mathbb{E}\left[U_{t} U_{t}^{\top}\right], \quad n^{-1} \mathbf{U}^{\top} \mathbf{U} \rightarrow \mathbb{E}\left[U U^{\top}\right]
$$




$$
d^{-1}\left(\mathbf{V}^{t}\right)^{\top} \mathbf{V}^{t} \rightarrow \mathbb{E}\left[V_{t} V_{t}^{\top}\right], \quad d^{-1}\left(\mathbf{V}^{t}\right)^{\top} \mathbf{V} \rightarrow \mathbb{E}\left[V_{t} V^{\top}\right]=\mathbb{E}\left[V_{t} V_{t}^{\top}\right], \quad d^{-1} \mathbf{V}^{\top} \mathbf{V} \rightarrow \mathbb{E}\left[V V^{\top}\right]
$$

where $\mathbb{E}\left[U_{t} U_{t}^{\top}\right]=S^{-1 / 2} Q_{t+1} S^{-1 / 2}$ and $\mathbb{E}\left[V_{t} V_{t}^{\top}\right]=S^{-1 / 2} \bar{Q}_{t} S^{-1 / 2}$. Together with the consistency $\hat{S} \rightarrow S$,

$$
\frac{1}{n d}\left\|\mathbf{U}^{t} \hat{S}\left(\mathbf{V}^{t}\right)^{\top}-\mathbf{U} S \mathbf{V}^{\top}\right\|_{F}^{2} \rightarrow \operatorname{Tr} \mathbb{E}\left[U U^{\top} S V V^{\top} S\right]-\operatorname{Tr} \bar{Q}_{t} Q_{t+1}
$$

By Proposition 5.6, we have $\operatorname{Tr} \bar{Q}_{t} Q_{t+1}=\operatorname{Tr} \bar{Q} Q+o_{t}(1)$ for the unique fixed point $(\bar{Q}, Q)$ of $(5.3)$, establishing the result.

\section{E Details of simulations and data analyses}

\section{E.1 Details of EB-PCA.}

A software implementation of EB-PCA is publicly available at https://github.com/TraceyZhong/ EBPCA.

In our implementation, we take $\mathcal{P}$ as the class of all probability distributions on $\mathbb{R}^{k}$, and approximate this class $\mathcal{P}$ using a discrete support. We apply the "exemplar method" of [LG08] and take the support points to be

$$
\left\{z_{1}, \ldots, z_{n}\right\}=\left\{M^{-1} x_{1}, \ldots, M^{-1} x_{n}\right\}
$$

This is motivated by the observation that these values should provide a fine grid that covers the high density regions under any true prior $\pi_{*} \in \mathcal{P}$. This grid automatically adapts to these high density regions, and the number of support points is independent of the dimension $k$. Thus we express

$$
\pi=\sum_{i=1}^{n} w_{i} \delta_{z_{i}}=\sum_{i=1}^{n} w_{i} \delta_{M^{-1} x_{i}}
$$

and maximize (2.8) over the probability weights $w_{1}, \ldots, w_{n}$.

The resulting maximization problem is concave over the weights $w_{1}, \ldots, w_{n}$. We solved this maximization using the generic interior point solver implemented in MOSEK. When analyzing real data of high dimension, we set the maximum number of prior support points to be 2000 to reduce the computational cost. More specifically, when either dimension ( $n$ or $d$ ) exceeded 2000, we drew a random subsample of size 2000 to be the prior support points.

\section{E.2 Details of simulations and applications}

Simulation details. To provide a direct comparison between mean-field VB and EB-PCA, we used the same NPMLE procedure in the two methods. EB-PCA, oracle Bayes AMP, and EBMF were all run for 10 iterations, which was sufficient for convergence.

For spca, we used the implementation in the $\mathrm{R}$ package elasticnet. We tested spca with a range of sparsity tuning parameter between 0.025 and 0.175 and report the best results.

Genotype data pre-processing. For the 1000 Genomes Project, we used the Phase III genotypes publicly available at https: //www . internationalgenome org. For the International HapMap Project, we used the third phase genotype data available at https://www . sanger.ac.uk/resources/ downloads/human/hapmap3.html.

For both 1000 Genomes and HapMap3, we used Plink(v1.90b6.9) to retain only common variants with minor allele frequency $>0.1$, and generated a set of such variants in approximate 
linkage disequilibrium (--indep 505 1.5). This yielded 466,431 SNPs for 1000 Genomes and 142,185 SNPs for HapMap3. For 1000 Genomes, we chose a random subset of 100,000 SNPs and used this to compute the ground truth. For HapMap3, we used all 142,185 selected SNPs to compute the ground truth.

Gene expression data pre-processing. We used raw PBMC single-cell data from 10X Genomics, publicly available at https://cf.10xgenomics.com/samples/cell/pbmc3k/pbmc3k_filtered_ gene_bc_matrices.tar.gz. We performed quality control following https://satijalab.org/ seurat/v3.2/pbmc3k_tutorial.html and applied an iterative procedure to clean the gene expression count matrix. We first removed genes with no variation across cells. We then centered and scaled the counts for each gene, and computed sample PCs. We used the PCs to identify outlier cells, and repeated this procedure with outliers removed. In total, we removed 12 cells and 3 genes in this cleaning step.

General pre-processing. In all data examples, we centered and scaled the samples (SNPs for genotype data, and genes for expression data) before applying either PCA or EB-PCA. This reduced the influence of single SNPs or single genes on the PCs, and improved their interpretability.

Accuracy of the noise model. Figure 8 displays a scree plot of all singular values, and a histogram of all bulk singular values, for each of the three data examples to which EB-PCA was applied. The distributions of singular values predicted by the square-root of the Marcenko-Pastur law, corresponding to the modeling assumption $w_{i j} \sim \mathcal{N}(0,1 / n)$, are overlaid on the histograms. We observe a near-perfect fit for the 1000 Genomes example, suggesting that a model of independent noise entries with homoscedastic variance may be quite accurate for the subsampled genotype data. The fit to the HapMap3 and the single-cell gene expression data are not as close, indicating that the noise model $w_{i j} \sim \mathcal{N}(0,1 / n)$ is a rougher approximation for these data, and suggesting the possibility for further improvement using a method developed around more general models of correlated noise.

Re-estimation of priors. Figure 7 compares estimation accuracy with and without re-estimating the priors $\bar{\pi}_{*}$ and $\pi_{*}$ after the first iteration, as discussed in Remark 2.3, on the 1000 Genomes example. For both subsample sizes of 100 and 1000 SNPs, the difference in estimation error between these approaches is minimal.

The runtime for EB-PCA in our implementation is dominated by the NPMLE computation. (For subsamples of 1000 SNPs, total runtime for 5 EB-PCA iterations was 53 seconds using the NPMLE update in each iteration, compared to 11 seconds using only the NPMLE in the first iteration.) These observations suggest that re-estimating the priors may be avoided without compromising accuracy, if computational cost is a concern.

Contribution of iterative refinement. Figure 7 depicts also the estimation errors across EBPCA iterations, on the same 1000 Genomes example. The decrease in error from Iteration 0 (sample $\mathrm{PCs}$ ) to Iteration 1 reflects the initial denoising step, and subsequent decreases in error indicate gains from iterative refinement using AMP. As discussed in the Main Text, for this data example, gains in accuracy for EB-PCA resulted mostly from the initial denoising, and EB-PCA typically converged within 1-2 iterations. This is in contrast to the simulated examples of Figures 3(c) and 9(c) for weaker signal strengths, where both initial denoising and iterative refinement contribute to the improved accuracy of EB-PCA. 
(a)

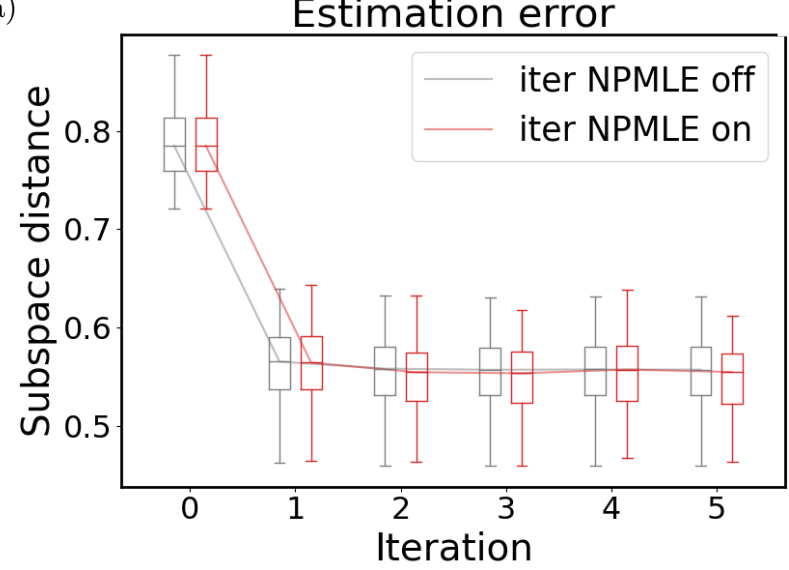

(b)

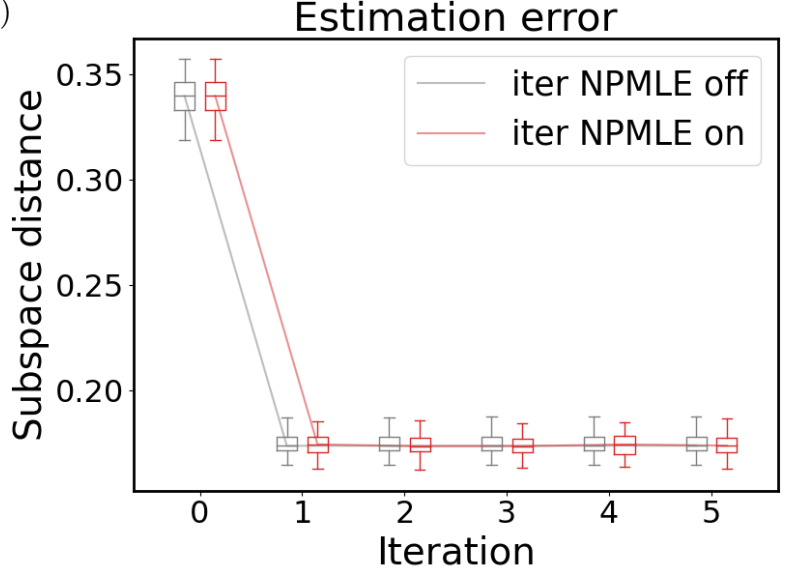

Figure 7: Estimation errors for EB-PCA with and without iterative updates of priors using NPMLE. (a)-(b) Comparison on 50 random subsamples of 100 SNPs or 1000 SNPs from the 1000 Genomes project.

Table 2: PC estimation errors for two simulated bivariate priors

\begin{tabular}{c|ccc|ccc}
\hline & \multicolumn{3}{|c|}{ Concentric circle } & \multicolumn{3}{c}{ Three-point mixture } \\
\hline Error & PC1 & PC2 & Joint & PC1 & PC2 & Joint \\
\hline \hline PCA & $.25(.0060)$ & $.52(.014)$ & $.41(.0080)$ & $.25(.0064)$ & $.52(.016)$ & $.40(.011)$ \\
Marginal EB-PCA & $.23(.0068)$ & $.47(.014)$ & $.37(.0088)$ & $.081(.020)$ & $.30(.027)$ & $.22(.019)$ \\
Joint EB-PCA & $.22(.0065)$ & $.37(.019)$ & $.30(.011)$ & $.046(.018)$ & $.080(.033)$ & $.067(.025)$ \\
\hline
\end{tabular}

Table 3: PC estimation errors on subsampled genotype matrices from the HapMap3

\begin{tabular}{l|c|ccccc}
\hline & Error & PC1 & PC2 & PC3 & PC4 & Joint \\
\hline \hline \multirow{2}{*}{1000 SNPs } & PCA & $.11(.0069)$ & $.16(.0059)$ & $.45(.060)$ & $.54(.094)$ & $.51(.098)$ \\
& EB-PCA & $.064(.012)$ & $.084(.0091)$ & $.33(.088)$ & $.37(.14)$ & $.36(.14)$ \\
\hline \multirow{2}{*}{5000 SNPs } & PCA & $.049(.0029)$ & $.071(.0023)$ & $.22(.043)$ & $.27(.051)$ & $.25(.043)$ \\
& EB-PCA & $.032(.0044)$ & $.043(.0038)$ & $.17(.052)$ & $.20(.068)$ & $.17(.053)$ \\
\hline \multirow{2}{*}{10000 SNPs } & PCA & $.034(.0023)$ & $.049(.0020)$ & $.15(.021)$ & $.18(.029)$ & $.17(.027)$ \\
& EB-PCA & $.024(.0027)$ & $.032(.0026)$ & $.12(.027)$ & $.14(.035)$ & $.13(.033)$ \\
\hline
\end{tabular}


(a)
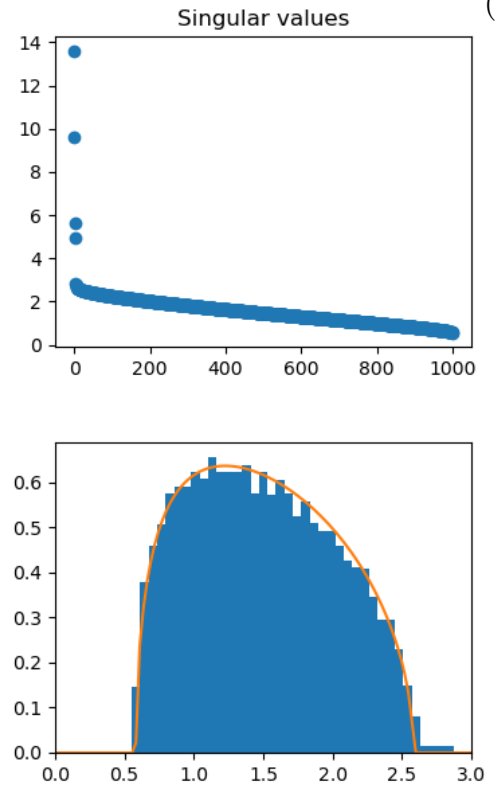

(b)
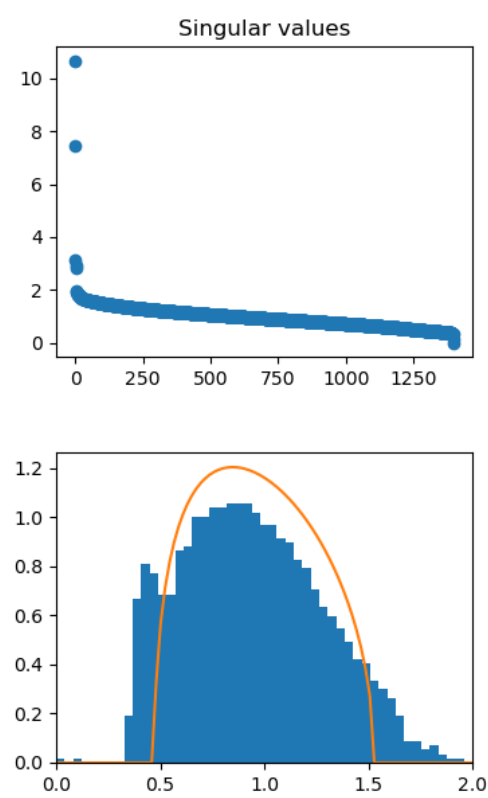

(c)
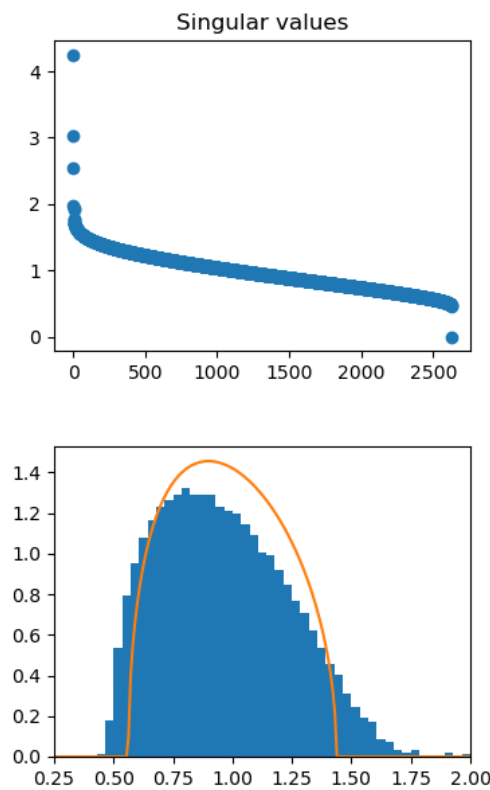

Figure 8: Scree plots and histograms of the singular values for (a) the matrix of 1000 subsampled SNPs from the 1000 Genomes Project, corresponding to Figure 1, (b) the matrix of 5000 subsampled SNPs from the HapMap3, corresponding to Figure 5, and (c) the matrix of all 13,711 gene expressions from the PBMC single-cell RNA-seq data, corresponding to Figure 6. A theoretical bulk distribution for the singular values, predicted by the Marcenko-Pastur law under the noise model $w_{i j} \stackrel{i i d}{\sim} \mathcal{N}(0,1 / n)$, is overlaid on the histograms.

(a)
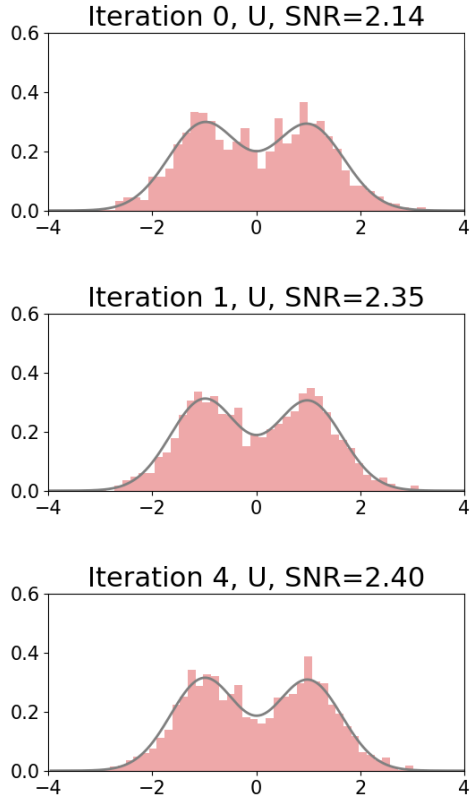

(b)

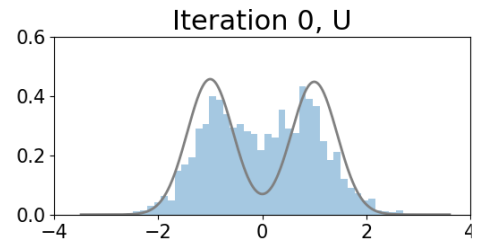

(c)
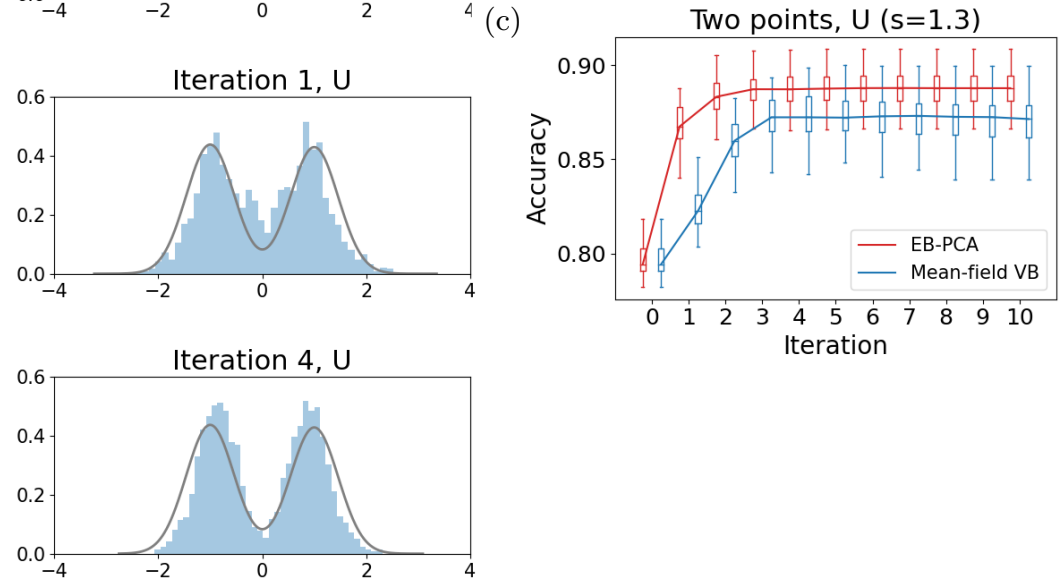

Figure 9: Same comparisons as in Figure 3, with a two point Bernoulli $\{+1,-1\}$ prior and overlaid convolution densities Bernoulli $\{+1,-1\} * \mathcal{N}\left(0, \bar{\sigma}_{t}^{2} / \bar{\mu}_{t}^{2}\right)$. 


\section{References}

[AFUZ19] Fabrizio Antenucci, Silvio Franz, Pierfrancesco Urbani, and Lenka Zdeborová. Glassy nature of the hard phase in inference problems. Physical Review X, 9(1):011020, 2019.

[AK18] Ahmed El Alaoui and Florent Krzakala. Estimation in the spiked Wigner model: A short proof of the replica formula. In IEEE International Symposium on Information Theory, pages $1874-1878,2018$.

[AW08] Arash A Amini and Martin J Wainwright. High-dimensional analysis of semidefinite relaxations for sparse principal components. In IEEE International Symposium on Information Theory, pages $2454-2458,2008$.

[BBAP05] Jinho Baik, Gérard Ben Arous, and Sandrine Péché. Phase transition of the largest eigenvalue for nonnull complex sample covariance matrices. The Annals of Probability, 33(5):1643-1697, 2005.

$\left[\mathrm{BDM}^{+} 16\right]$ Jean Barbier, Mohamad Dia, Nicolas Macris, Florent Krzakala, Thibault Lesieur, and Lenka Zdeborová. Mutual information for symmetric rank-one matrix estimation: A proof of the replica formula. In Advances in Neural Information Processing Systems, pages 424-432, 2016.

[BDW21] Zhigang Bao, Xiucai Ding, and Ke Wang. Singular vector and singular subspace distribution for the matrix denoising model. The Annals of Statistics, 49(1):370-392, 2021.

[BDWW20] Zhigang Bao, Xiucai Ding, Jingming Wang, and Ke Wang. Statistical inference for principal components of spiked covariance matrix. arXiv preprint arXiv:2008.11903, 2020.

[BGN11] Florent Benaych-Georges and Raj Rao Nadakuditi. The eigenvalues and eigenvectors of finite, low rank perturbations of large random matrices. Advances in Mathematics, 227(1):494-521, 2011.

[BGN12] Florent Benaych-Georges and Raj Rao Nadakuditi. The singular values and vectors of low rank perturbations of large rectangular random matrices. Journal of Multivariate Analysis, 111:120-135, 2012.

[BJNP13] Aharon Birnbaum, Iain M Johnstone, Boaz Nadler, and Debashis Paul. Minimax bounds for sparse PCA with noisy high-dimensional data. The Annals of Statistics, 41(3):1055, 2013.

[BKYY16] Alex Bloemendal, Antti Knowles, Horng-Tzer Yau, and Jun Yin. On the principal components of sample covariance matrices. Probability Theory and Related Fields, 164(1-2):459-552, 2016.

[BLM15] Mohsen Bayati, Marc Lelarge, and Andrea Montanari. Universality in polytope phase transitions and message passing algorithms. The Annals of Applied Probability, 25(2):753-822, 2015.

[BM19] Jean Barbier and Nicolas Macris. The adaptive interpolation method: A simple scheme to prove replica formulas in Bayesian inference. Probability Theory and Related Fields, 174(34):1133-1185, 2019.

[Böh99] Dankmar Böhning. Computer-assisted analysis of mixtures and applications: meta-analysis, disease mapping and others, volume 81. CRC press, 1999.

[BS06] Jinho Baik and Jack W Silverstein. Eigenvalues of large sample covariance matrices of spiked population models. Journal of multivariate analysis, 97(6):1382-1408, 2006.

[BSL92] Dankmar Bohning, Peter Schlattmann, and Bruce Lindsay. Computer-assisted analysis of mixtures (CA MAN): statistical algorithms. Biometrics, pages 283-303, 1992.

[BY08] Zhidong Bai and Jianfeng Yao. Central limit theorems for eigenvalues in a spiked population model. Annales de l'IHP Probabilités et statistiques, 44(3):447-474, 2008.

[BY12] Zhidong Bai and Jianfeng Yao. On sample eigenvalues in a generalized spiked population model. Journal of Multivariate Analysis, 106:167-177, 2012. 
[Cap18] Mireille Capitaine. Limiting eigenvectors of outliers for spiked information-plus-noise type matrices. In Séminaire de Probabilités XLIX, pages 119-164. Springer, 2018.

[CCS10] Jian-Feng Cai, Emmanuel J Candès, and Zuowei Shen. A singular value thresholding algorithm for matrix completion. SIAM Journal on Optimization, 20(4):1956-1982, 2010.

[CDM18] Mireille Capitaine and Catherine Donati-Martin. Non universality of fluctuations of outlier eigenvectors for block diagonal deformations of Wigner matrices. arXiv preprint arXiv:1807.07773, 2018.

[CDMFF11] Mireille Capitaine, Catherine Donati-Martin, Delphine Féral, and Maxime Février. Free convolution with a semicircular distribution and eigenvalues of spiked deformations of Wigner matrices. Electronic Journal of Probability, 16:1750-1792, 2011.

[Cha15] Sourav Chatterjee. Matrix estimation by universal singular value thresholding. The Annals of Statistics, 43(1):177-214, 2015.

[CJ95] Jorge Cadima and Ian T Jolliffe. Loading and correlations in the interpretation of principal compenents. Journal of Applied Statistics, 22(2):203-214, 1995.

[CL21] Wei-Kuo Chen and Wai-Kit Lam. Universality of approximate message passing algorithms. Electronic Journal of Probability, 26:1-44, 2021.

[CMW13] T Tony Cai, Zongming Ma, and Yihong Wu. Sparse PCA: Optimal rates and adaptive estimation. The Annals of Statistics, 41(6):3074-3110, 2013.

[DAM17] Yash Deshpande, Emmanuel Abbe, and Andrea Montanari. Asymptotic mutual information for the balanced binary stochastic block model. Information and Inference: A Journal of the IMA, 6(2):125-170, 2017.

[dGJL05] Alexandre d'Aspremont, Laurent E Ghaoui, Michael I Jordan, and Gert R Lanckriet. A direct formulation for sparse PCA using semidefinite programming. In Advances in neural information processing systems, pages 41-48, 2005.

[Din20] Xiucai Ding. High dimensional deformed rectangular matrices with applications in matrix denoising. Bernoulli, 26(1):387-417, 2020.

[DM14] Yash Deshpande and Andrea Montanari. Information-theoretically optimal sparse PCA. In IEEE International Symposium on Information Theory, pages 2197-2201, 2014.

[DMM09] David L Donoho, Arian Maleki, and Andrea Montanari. Message-passing algorithms for compressed sensing. Proceedings of the National Academy of Sciences, 106(45):18914-18919, 2009.

[DY21] Xiucai Ding and Fan Yang. Spiked separable covariance matrices and principal components. The Annals of Statistics, 49(2):1113-1138, 2021.

[Efr12] Bradley Efron. Large-scale inference: Empirical Bayes methods for estimation, testing, and prediction, volume 1. Cambridge University Press, 2012.

[Fan20] Zhou Fan. Approximate message passing algorithms for rotationally invariant matrices. arXiv preprint arXiv:2008.11892, 2020.

[FD16] Long Feng and Lee H Dicker. Approximate nonparametric maximum likelihood inference for mixture models via convex optimization. arXiv preprint arXiv:1606.02011, 2016.

[FLM13] Jianqing Fan, Yuan Liao, and Martina Mincheva. Large covariance estimation by thresholding principal orthogonal complements. Journal of the Royal Statistical Society. Series B, Statistical methodology, 75(4), 2013.

[FMM21] Zhou Fan, Song Mei, and Andrea Montanari. TAP free energy, spin glasses and variational inference. The Annals of Probability, 49(1):1-45, 2021.

[GD17] Matan Gavish and David L Donoho. Optimal shrinkage of singular values. IEEE Transactions on Information Theory, 63(4):2137-2152, 2017. 
[GJM19] Behrooz Ghorbani, Hamid Javadi, and Andrea Montanari. An instability in variational inference for topic models. In International Conference on Machine Learning, pages 2221-2231, 2019.

[GvdV01] Subhashis Ghosal and Aad W van der Vaart. Entropies and rates of convergence for maximum likelihood and Bayes estimation for mixtures of normal densities. Annals of Statistics, pages 1233-1263, 2001.

[Int10] International HapMap 3 Consortium. Integrating common and rare genetic variation in diverse human populations. Nature, 467(7311):52, 2010.

[Jew82] Nicholas P Jewell. Mixtures of exponential distributions. The Annals of Statistics, 10(2):479484, 1982.

[JL09] Iain M Johnstone and Arthur Yu Lu. On consistency and sparsity for principal components analysis in high dimensions. Journal of the American Statistical Association, 104(486):682-693, 2009.

[JM09] Sungkyu Jung and J Stephen Marron. PCA consistency in high dimension, low sample size context. The Annals of Statistics, 37(6B):4104-4130, 2009.

[Joh01] Iain M Johnstone. On the distribution of the largest eigenvalue in principal components analysis. The Annals of Statistics, pages 295-327, 2001.

[JP18] Iain M Johnstone and Debashis Paul. PCA in high dimensions: An orientation. Proceedings of the IEEE, 106(8):1277-1292, 2018.

[JTU03] Ian T Jolliffe, Nickolay T Trendafilov, and Mudassir Uddin. A modified principal component technique based on the LASSO. Journal of Computational and Graphical Statistics, 12(3):531$547,2003$.

[JZ09] Wenhua Jiang and Cun-Hui Zhang. General maximum likelihood empirical Bayes estimation of normal means. The Annals of Statistics, 37(4):1647-1684, 2009.

[Kab03] Yoshiyuki Kabashima. A CDMA multiuser detection algorithm on the basis of belief propagation. Journal of Physics A: Mathematical and General, 36(43):11111, 2003.

[KKM $\left.{ }^{+} 16\right]$ Yoshiyuki Kabashima, Florent Krzakala, Marc Mézard, Ayaka Sakata, and Lenka Zdeborová. Phase transitions and sample complexity in Bayes-optimal matrix factorization. IEEE Transactions on Information Theory, 62(7):4228-4265, 2016.

[KM14] Roger Koenker and Ivan Mizera. Convex optimization, shape constraints, compound decisions, and empirical Bayes rules. Journal of the American Statistical Association, 109(506):674-685, 2014.

$\left[\mathrm{KMS}^{+} 12\right] \quad$ Florent Krzakala, Marc Mézard, François Sausset, YF Sun, and Lenka Zdeborová. Statisticalphysics-based reconstruction in compressed sensing. Physical Review X, 2(2):021005, 2012.

[KRFU14] Ulugbek S Kamilov, Sundeep Rangan, Alyson K Fletcher, and Michael Unser. Approximate message passing with consistent parameter estimation and applications to sparse learning. IEEE Transactions on Information Theory, 60(5):2969-2985, 2014.

[KW56] Jack Kiefer and Jacob Wolfowitz. Consistency of the maximum likelihood estimator in the presence of infinitely many incidental parameters. The Annals of Mathematical Statistics, 27(4):887-906, 1956.

[KY13] Antti Knowles and Jun Yin. The isotropic semicircle law and deformation of Wigner matrices. Communications on Pure and Applied Mathematics, 66(11):1663-1749, 2013.

[KY14] Antti Knowles and Jun Yin. The outliers of a deformed Wigner matrix. The Annals of Probability, 42(5):1980-2031, 2014.

[Lai78] Nan Laird. Nonparametric maximum likelihood estimation of a mixing distribution. Journal of the American Statistical Association, 73(364):805-811, 1978. 
[LG08] Danial Lashkari and Polina Golland. Convex clustering with exemplar-based models. In $A d$ vances in Neural Information Processing Systems, pages 825-832, 2008.

[Lin83a] Bruce G Lindsay. The geometry of mixture likelihoods: a general theory. The Annals of Statistics, 11(1):86-94, 1983.

[Lin83b] Bruce G Lindsay. The geometry of mixture likelihoods, part II: the exponential family. The Annals of Statistics, 11(3):783-792, 1983.

[Lin95] Bruce G Lindsay. Mixture models: theory, geometry and applications. Institute of Mathematical Statistics, 1995.

[LKZ15a] Thibault Lesieur, Florent Krzakala, and Lenka Zdeborová. MMSE of probabilistic low-rank matrix estimation: Universality with respect to the output channel. In 2015 53rd Annual Allerton Conference on Communication, Control, and Computing (Allerton), pages 680-687. IEEE, 2015.

[LKZ15b] Thibault Lesieur, Florent Krzakala, and Lenka Zdeborová. Phase transitions in sparse PCA. In 2015 IEEE International Symposium on Information Theory (ISIT), pages 1635-1639. IEEE, 2015.

[LM19] Marc Lelarge and Léo Miolane. Fundamental limits of symmetric low-rank matrix estimation. Probability Theory and Related Fields, 173(3-4):859-929, 2019.

[LR93] Bruce G Lindsay and Kathryn Roeder. Uniqueness of estimation and identifiability in mixture models. Canadian Journal of Statistics, 21(2):139-147, 1993.

[Lu02] Arthur Yu Lu. Sparse principal component analysis for functional data. PhD thesis, Stanford University, 2002.

[LW12] Olivier Ledoit and Michael Wolf. Nonlinear shrinkage estimation of large-dimensional covariance matrices. The Annals of Statistics, 40(2):1024-1060, 2012.

[Ma13] Zongming Ma. Sparse principal component analysis and iterative thresholding. The Annals of Statistics, 41(2):772-801, 2013.

[Mes08] Xavier Mestre. On the asymptotic behavior of the sample estimates of eigenvalues and eigenvectors of covariance matrices. IEEE Transactions on Signal Processing, 56(11):5353-5368, 2008.

[Mio17] Léo Miolane. Fundamental limits of low-rank matrix estimation: the non-symmetric case. arXiv preprint arXiv:1702.00473, 2017.

[MR15] Andrea Montanari and Emile Richard. Non-negative principal component analysis: Message passing algorithms and sharp asymptotics. IEEE Transactions on Information Theory, 62(3):1458-1484, 2015.

[MT13] Ryosuke Matsushita and Toshiyuki Tanaka. Low-rank matrix reconstruction and clustering via approximate message passing. In Advances in Neural Information Processing Systems, pages 917-925, 2013.

[MV21] Andrea Montanari and Ramji Venkataramanan. Estimation of low-rank matrices via approximate message passing. The Annals of Statistics, 49(1):321-345, 2021.

[Nad08] Boaz Nadler. Finite sample approximation results for principal component analysis: A matrix perturbation approach. The Annals of Statistics, 36(6):2791-2817, 2008.

[Nad14] Raj Rao Nadakuditi. Optshrink: An algorithm for improved low-rank signal matrix denoising by optimal, data-driven singular value shrinkage. IEEE Transactions on Information Theory, 60(5):3002-3018, 2014.

[Pau07] Debashis Paul. Asymptotics of sample eigenstructure for a large dimensional spiked covariance model. Statistica Sinica, 17(4):1617-1642, 2007. 
[PW20] Yury Polyanskiy and Yihong Wu. Self-regularizing property of nonparametric maximum likelihood estimator in mixture models. arXiv preprint arXiv:2008.08244, 2020.

[Ran11] Sundeep Rangan. Generalized approximate message passing for estimation with random linear mixing. In IEEE International Symposium on Information Theory Proceedings, pages 2168$2172,2011$.

[RF12] Sundeep Rangan and Alyson K Fletcher. Iterative estimation of constrained rank-one matrices in noise. In IEEE International Symposium on Information Theory Proceedings, pages 12461250, 2012.

[Rob50] Herbert Robbins. A generalization of the method of maximum likelihood: Estimating a mixing distribution (abstract). Annals of Mathematical Statistics, 21(2):314-315, 1950.

[Rob56] Herbert Robbins. An empirical Bayes approach to statistics. In Proceedings of the Third Berkeley Symposium on Mathematical Statistics and Probability, Volume 1: Contributions to the Theory of Statistics. The Regents of the University of California, 1956.

[RPD18] Galen Reeves, Henry D Pfister, and Alex Dytso. Mutual information as a function of matrix snr for linear gaussian channels. In IEEE International Symposium on Information Theory (ISIT), pages 1754-1758, 2018.

[SG20] Sujayam Saha and Adityanand Guntuboyina. On the nonparametric maximum likelihood estimator for Gaussian location mixture densities with application to Gaussian denoising. The Annals of Statistics, 48(2):738-762, 2020.

[Sim76] Leopold Simar. Maximum likelihood estimation of a compound Poisson process. The Annals of Statistics, pages 1200-1209, 1976.

[SN13] Andrey A Shabalin and Andrew B Nobel. Reconstruction of a low-rank matrix in the presence of Gaussian noise. Journal of Multivariate Analysis, 118:67-76, 2013.

[SSM13] Dan Shen, Haipeng Shen, and James Stephen Marron. Consistency of sparse PCA in high dimension, low sample size contexts. Journal of Multivariate Analysis, 115:317-333, 2013.

[The15] The 1000 Genomes Project Consortium. A global reference for human genetic variation. Nature, 526(7571):68-74, 2015.

[VCLR13] Vincent Q Vu, Juhee Cho, Jing Lei, and Karl Rohe. Fantope projection and selection: A near-optimal convex relaxation of sparse PCA. In Advances in Neural Information Processing Systems, pages 2670-2678, 2013.

[Ver18] Roman Vershynin. High-dimensional probability: An introduction with applications in data science, volume 47. Cambridge university press, 2018.

[VLG13] Charles F Van Loan and Gene H Golub. Matrix computations. Johns Hopkins University Press Baltimore, 4th edition, 2013.

[VS11] Jeremy Vila and Philip Schniter. Expectation-maximization Bernoulli-Gaussian approximate message passing. In 2011 Conference Record of the Forty Fifth Asilomar Conference on Signals, Systems and Computers (ASILOMAR), pages 799-803. IEEE, 2011.

[VS13] Jeremy P Vila and Philip Schniter. Expectation-maximization Gaussian-mixture approximate message passing. IEEE Transactions on Signal Processing, 61(19):4658-4672, 2013.

[WF17] Weichen Wang and Jianqing Fan. Asymptotics of empirical eigenstructure for high dimensional spiked covariance. The Annals of Statistics, 45(3):1342, 2017.

[WS21] Wei Wang and Matthew Stephens. Empirical bayes matrix factorization. Journal of Machine Learning Research, 22(120):1-40, 2021.

[Yan19] Fan Yang. Edge universality of separable covariance matrices. Electronic Journal of Probability, 24, 2019. 
[Zha09] Cun-Hui Zhang. Generalized maximum likelihood estimation of normal mixture densities. Statistica Sinica, 19(3):1297-1318, 2009.

[ZHT06] Hui Zou, Trevor Hastie, and Robert Tibshirani. Sparse principal component analysis. Journal of Computational and Graphical Statistics, 15(2):265-286, 2006. 